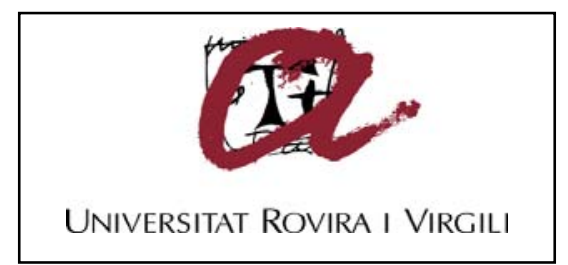

\title{
COMPACT MODELING OF INTRINSIC CAPACITANCES IN DOUBLE-GATE TUNNEL-FETS
}

\author{
Atieh Farokhnejad
}

\begin{abstract}
ADVERTIMENT. L'accés als continguts d'aquesta tesi doctoral i la seva utilització ha de respectar els drets de la persona autora. Pot ser utilitzada per a consulta o estudi personal, així com en activitats o materials d'investigació i docència en els termes establerts a l'art. 32 del Text Refós de la Llei de Propietat Intel.lectual (RDL 1/1996). Per altres utilitzacions es requereix l'autorització prèvia i expressa de la persona autora. En qualsevol cas, en la utilització dels seus continguts caldrà indicar de forma clara el nom i cognoms de la persona autora i el títol de la tesi doctoral. No s'autoritza la seva reproducció o altres formes d'explotació efectuades amb finalitats de lucre ni la seva comunicació pública des d'un lloc aliè al servei TDX. Tampoc s'autoritza la presentació del seu contingut en una finestra o marc aliè a TDX (framing). Aquesta reserva de drets afecta tant als continguts de la tesi com als seus resums i índexs.
\end{abstract}

ADVERTENCIA. El acceso a los contenidos de esta tesis doctoral y su utilización debe respetar los derechos de la persona autora. Puede ser utilizada para consulta o estudio personal, así como en actividades o materiales de investigación y docencia en los términos establecidos en el art. 32 del Texto Refundido de la Ley de Propiedad Intelectual (RDL 1/1996). Para otros usos se requiere la autorización previa y expresa de la persona autora. En cualquier caso, en la utilización de sus contenidos se deberá indicar de forma clara el nombre y apellidos de la persona autora y el título de la tesis doctoral. No se autoriza su reproducción u otras formas de explotación efectuadas con fines lucrativos ni su comunicación pública desde un sitio ajeno al servicio TDR. Tampoco se autoriza la presentación de su contenido en una ventana o marco ajeno a TDR (framing). Esta reserva de derechos afecta tanto al contenido de la tesis como a sus resúmenes e índices.

WARNING. Access to the contents of this doctoral thesis and its use must respect the rights of the author. It can be used for reference or private study, as well as research and learning activities or materials in the terms established by the 32nd article of the Spanish Consolidated Copyright Act (RDL 1/1996). Express and previous authorization of the author is required for any other uses. In any case, when using its content, full name of the author and title of the thesis must be clearly indicated. Reproduction or other forms of for profit use or public communication from outside TDX service is not allowed. Presentation of its content in a window or frame external to TDX (framing) is not authorized either. These rights affect both the content of the thesis and its abstracts and indexes. 
\#

TECHNISCHE HOCHSCHULE MITTELHESSEN

\section{Compact Modeling of Intrinsic Capacitances in Double-Gate Tunnel-FETs}

Atieh Farokhnejad

\section{$7^{2}$}

$d^{2} \psi$

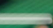

58

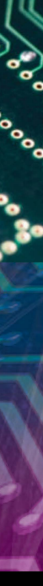

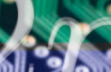

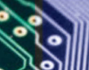

(2)
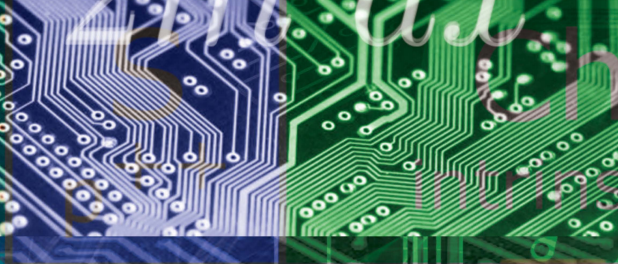

\section{\%.}
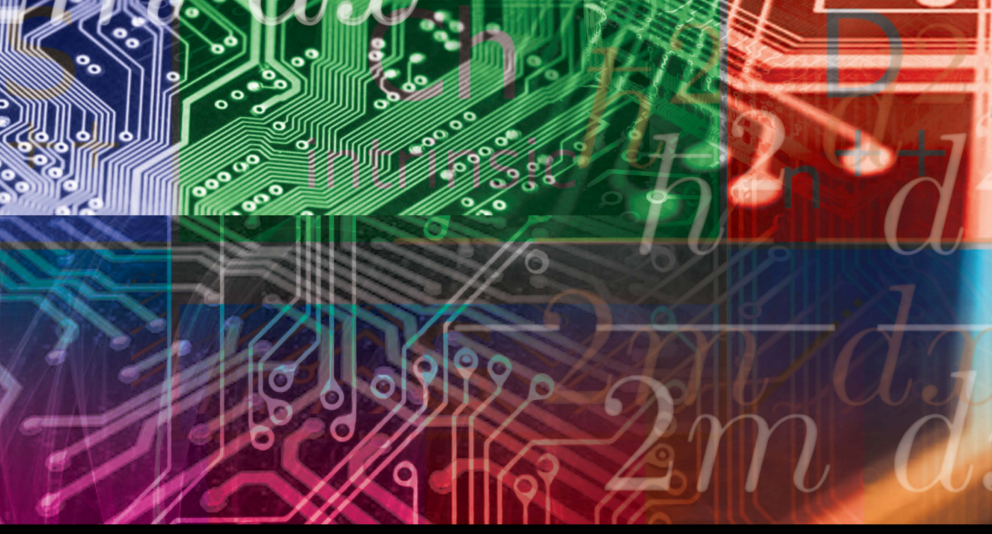

DOCTORAL THESIS 2020 


\section{UNIVERSITAT ROVIRA I VIRGILI}

COMPACT MODELING OF INTRINSIC CAPACITANCES IN DOUBLE-GATE TUNNEL-FETS

Atieh Farokhnejad 


\section{UNIVERSITAT ROVIRA I VIRGILI}

COMPACT MODELING OF INTRINSIC CAPACITANCES IN DOUBLE-GATE TUNNEL-FETS

Atieh Farokhnejad 


\section{UNIVERSITAT ROVIRA I VIRGILI}

COMPACT MODELING OF INTRINSIC CAPACITANCES IN DOUBLE-GATE TUNNEL-FETS

Atieh Farokhnejad 
Atieh Farokhnejad

Compact Modeling of Intrinsic Capacitances in Double-Gate Tunnel-FETs

\title{
DOCTORAL THESIS
}

Supervised by Prof. Dr. Benjamín Iñíguez

Associate Prof. Dr. François Lime

and Prof. Dr.-Ing. Alexander Kloes

Department of Electronic,

Electrical and Automatic Control Engineering

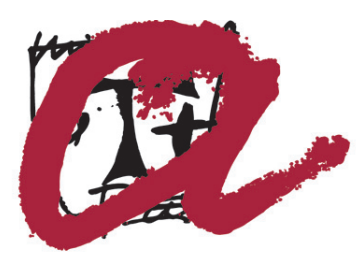

\section{UNIVERSITAT ROVIRA I VIRGILI}

\author{
Tarragona
}

2020 


\section{UNIVERSITAT ROVIRA I VIRGILI}

COMPACT MODELING OF INTRINSIC CAPACITANCES IN DOUBLE-GATE TUNNEL-FETS

Atieh Farokhnejad 


\section{Statement of Supervision}

\section{UNIVERSITAT ROVIRA I VIRGILI}

\section{Department of Electronic, Electric}

\section{and Automatic Engineering (DEEEA)}

Av. Paisos Catalans 26, Campus Sescelades

43007, Tarragona, Spain

Phone: +34 977558524

Fax: +34 977559605

I STATE that the present study, entitled: "Compact Modeling of Intrinsic Capacitances in Double-Gate Tunnel-FETs", presented by Atieh Farokhnejad for the award of the degree of the Doctor, has been carried out under my supervision at the Department of Electronic, Electrical and Automatic Control Engineering of this university, and that it fulfills all the requirements to be eligible for the European Doctorate Award.

Tarragona (Spain), June 4, 2020

Prof. Dr. Benjamín Iñíguez, Doctoral Thesis Supervisor

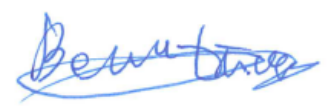

Associate Prof. Dr. François Lime, Doctoral Thesis Tutor

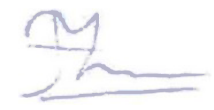

Prof. Dr.-Ing. Alexander Kloes, Doctoral Thesis Supervisor

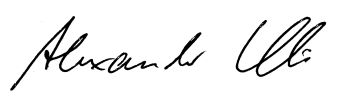




\section{UNIVERSITAT ROVIRA I VIRGILI}

COMPACT MODELING OF INTRINSIC CAPACITANCES IN DOUBLE-GATE TUNNEL-FETS

Atieh Farokhnejad 


\section{Statement of Authorship}

\section{\#IIHM}

TECHNISCHE HOCHSCHULE MITTELHESSEN

\section{Research Group Nanoelectronics / Device Modeling}

Wiesenstrasse 14

35390, Giessen, Germany

Phone: +49 641 309-1968

Fax: +49 641 309-2901

I STATE that this document has been composed by myself and describes my own work, unless otherwise acknowledged in the text. Parts that are direct quotes or paraphrases are identified as such. It has not been accepted in any previous application for a degree. All sources of information have been specifically acknowledged.

Giessen (Germany), June 4, 2020

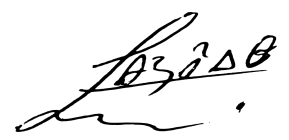

Atieh Farokhnejad, M. Sc. 


\section{UNIVERSITAT ROVIRA I VIRGILI}

COMPACT MODELING OF INTRINSIC CAPACITANCES IN DOUBLE-GATE TUNNEL-FETS

Atieh Farokhnejad 


\section{Acknowledgments}

Dear Prof. Dr.-Ing. Alexander Kloes, words do little justice in acknowledgment all you have done for me. I would like to thank you fervently for being ever so supportive and compassionate towards by needs. Being a member of your group is an honor, nay, a privilege to say the least. You were always an advocate of us enjoying what we did and to that end, I would like to mention that working under your guidance was a most enjoyable enterprise.

Dear Prof. Dr. Benjamín Iñíguez, I would like to thank you for your helpful comments and guidance which served well bringing my thesis to fruition.

Dear Prof. Dr. François Lime, thanks for your support and supervision. Your comments have helped me to gain a better and deeper insight into my study.

Dear Prof. Dr. Qing-Tai Zhao, thank you very much for your noteworthy contribution and providing me with the valuable data.

Dear Prof. Dr. Siegfried Mantl, many thanks for the collaboration that made my visit to FZ Juelich possible and for being such a nice host during my visit.

Dear Dr. Eduardo Pérez Diez, thanks ever so for your helpful advice throughout my study. I have learned a lot from you which stood me on good stead.

I would like to thank my colleagues Dr. Michael Gräf, Dr. Fabian Hosenfeld, Dr. Fabian Horst, Jakob Prüfer, Jakob Leise and Christian Römer, for their ever present support. The knowledge that you have imparted will forever serve me well. Our beautiful and bountiful memories will never fail to brighten my day.

I would like to thank my ever loving Parents for their stalwart companionship, especially during times of great crises and equal dejection. You always rallied my spirits ever so famously with your unequaled warmth and words of wisdom. I would also like to thank my ever loving brothers for their altruistic nature which always kept me out of mischief. My dearest Tahereh, I would love nothing more than to call you my foremost and sincerest friend. You were always the Virgil to my Dante.

This project was supported by the German Federal Ministry of Education and Research under contract No. FKZ13FH010IX5 and the Spanish Ministry of Economy and Competitiveness through project GREENSENSE (TEC2015-67883-R). I would also like to thank AdMOS GmbH for their support.

Last but not the least I would like to thank FZ Juelich, PGI9-IT and LSI/PSI/USP, 
University of Sao Paulo for the cooperation and providing the measurement data. 


\section{UNIVERSITAT ROVIRA I VIRGILI}

COMPACT MODELING OF INTRINSIC CAPACITANCES IN DOUBLE-GATE TUNNEL-FETS

Atieh Farokhnejad 
COMPACT MODELING OF INTRINSIC CAPACITANCES IN DOUBLE-GATE TUNNEL-FETS

Atieh Farokhnejad

to you my beautiful Mama,

to you my lovely Dad, no matter how hard or easy, how far or close, you never gave up on me... 


\section{UNIVERSITAT ROVIRA I VIRGILI}

COMPACT MODELING OF INTRINSIC CAPACITANCES IN DOUBLE-GATE TUNNEL-FETS

Atieh Farokhnejad 
COMPACT MODELING OF INTRINSIC CAPACITANCES IN DOUBLE-GATE TUNNEL-FETS

Atieh Farokhnejad 


\section{Contents}

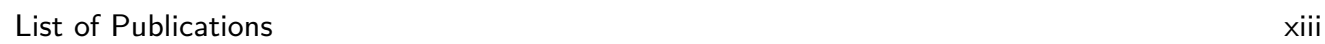

List of Symbols $\quad$ xix

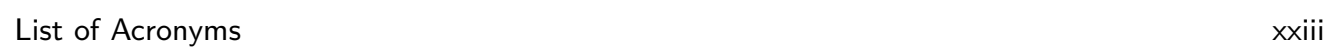

1 Introduction $\quad 1$

1.1 Why TFET? . . . . . . . . . . . . . . . . . . . . . . . . 1

1.2 Necessity of Device Simulation and Compact Model . . . . . . . . . . . . . . . 3

1.3 Outline of the Thesis . . . . . . . . . . . . . . . . . . . . . . 5

2 History of Microelectronics $\quad 6$

2.1 Mechanical Computers . . . . . . . . . . . . . . . . . . . . . . . . . 6

2.2 Electronic Computers . . . . . . . . . . . . . . . . . . . . . . . 7

2.3 Invention of Transistors . . . . . . . . . . . . . . . . . . . . . . . . . . 9

2.4 Moore's Law . . . . . . . . . . . . . . . . . . . . . . . . . . . . . . 10

3 Theoretical Backgrounds $\quad 12$

3.1 Semiconductor Materials . . . . . . . . . . . . . . . . . . . . . . . . . . . . 12

3.1.1 Fermi Distribution . . . . . . . . . . . . . . . . . . . . 12

3.1.2 Band structure .......................... . . . 13

3.1.3 Doped Semiconductors . . . . . . . . . . . . . . . . . . 15

3.2 Quantum Tunneling . . . . . . . . . . . . . . . . . . . . . . . . . 16

3.3 Tunneling Events . . . . . . . . . . . . . . . . . . . . . . . . . . . . . 17

3.4 Tunneling Probability . . . . . . . . . . . . . . . . . . . . 18

3.4.1 Rectangular Barrier . . . . . . . . . . . . . . . . . . . 19

3.4.2 Triangular Barrier ... . . . . . . . . . . . . . . . . . . . 21

3.5 Poisson's and Laplace's Equations . . . . . . . . . . . . . . . . . . . . . . . . . 24

3.6 Lambert's $W$ function . . . . . . . . . . . . . . . . . . . . . . . . . . . . . . . . 25 
4 TFET Essentials

4.1 Device Geometry . . . . . . . . . . . . . . . . . . . . . . 27

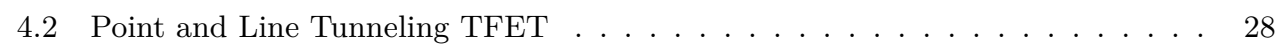

4.3 Working Principle . . . . . . . . . . . . . . . . . . . . 29

4.3 .1 Off-State . . . . . . . . . . . . . . . . . . . 29

4.3 .2 On-State . . . . . . . . . . . . . . . . . 30

4.3 .3 Ambipolar-State . . . . . . . . . . . . . . . . . . 31

4.4 Transfer Characteristics . . . . . . . . . . . . . . . . . . . 31

4.5 Output Characteristics . . . . . . . . . . . . . . . . . . . . 32

4.6 Capacitances in TFET . . . . . . . . . . . . . . . 33

5 Model Development 37

5.1 Charge Calculation . . . . . . . . . . . . . . . . . . . . . 37

5.2 Intrinsic Capacitance Model . . . . . . . . . . . . . . . . . . . . 40

5.3 Effect of Parasitic Elements on Capacitances . . . . . . . . . . . . . . . . . 42

6 Model Verification $\quad 45$

6.1 Model Verification by Comparison with TCAD Simulations . . . . . . . . . . 45

6.2 Model Verification by Comparison with Measurements . . . . . . . . . . . . . 48

6.2 .1 Planar TFET . . . . . . . . . . . . . . . . . . . . 48

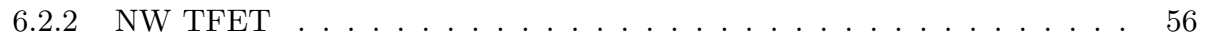

7 Circuit Simulation $\quad 60$

7.1 DG TFET-Based Circuits . . . . . . . . . . . . . . . . . . 60

7.2 SG Line Tunneling TFET-Based Circuits . . . . . . . . . . . . . . . . . 69

$\begin{array}{lll}8 \text { Conclusion } & 75\end{array}$

$\begin{array}{ll}\text { References } & 80\end{array}$ 


\section{List of Publications}

\section{Journals}

- A. Farokhnejad, M. Schwarz, F. Horst, B. Iñíguez, F. Lime, and A. Kloes, "Analytical Modeling of Capacitances in Tunnel-FETs Including the Effect of Schottky Barrier Contacts," in Solid-State Electronics, vol. 159, pp. 191-196, Sep. 2019.

DOI: 10.1016/j.sse.2019.03.062, Impact Factor: 1.492

\section{Conferences}

- A. Farokhnejad, F. Horst, B. Iñíguez, F. Lime, and A. Kloes, "Impact of On-Current on the Static and Dynamic Performance of TFET Inverters," accepted for publication at 2019 IEEE SOI-3D-Subthreshold Microelectronics Technology Unified Conference (S3S), San Jose, USA, Oct. 2019.

- A. Farokhnejad, F. Horst, B. Iñíguez, F. Lime, and A. Kloes, "Evaluation of Static/Transient Performance of TFET Inverter Regarding Device Parameters Using a Compact Model," accepted for publication at 49th European Solid-State Device Research Conference (ESSDERC), Kraków, Poland, Sept. 2019.

DOI: 10.1109/ESSDERC.2019.8901770

- A. Farokhnejad, M. Schwarz, M. Graef, F. Horst, B. Iñíguez, F. Lime and A. Kloes, "Effect of Schottky Barrier Contacts on Measured Capacitances in Tunnel-FETs," in 2018 Joint International EUROSOI Workshop and International Conference on Ultimate Integration on Silicon (EUROSOI-ULIS), Granada, Spain, Mar. 2018, pp. 1-4.

DOI: 10.1109/ULIS.2018.8354766

- A. Farokhnejad, M. Graef, F. Horst, C. Liu, Q.-T. Zhao, F. Lime and A. Kloes, "Compact Modeling of Intrinsic Capacitances in Double-Gate Tunnel-FETs," in 2017 Joint International EUROSOI Workshop and International Conference on Ultimate Integration on Silicon (EUROSOI-ULIS), Athens, Greece, Apr. 2017, pp. 140-143.

DOI: 10.1109/ULIS.2017.7962584 
- A. Farokhnejad, M. Graef and Alexander Kloes, "Wavelet-based calculation of the transmission coefficient for tunneling events in Tunnel-FETs," in 2015 22nd International Conference Mixed Design of Integrated Circuits \& Systems (MIXDES), Torun, Poland, Jun. 2015, pp. 210-215.

DOI: 10.1109/MIXDES.2015.7208512

\section{Co-Authorship}

- F. Horst, A. Farokhnejad, B. Iñíguez and A. Kloes, "Closed-Form Modeling Approach of Trap-Assisted Tunneling Current for Use in Compact TFET Models," in 2019 MIXDES - 26th International Conference "Mixed Design of Integrated Circuits and Systems", Rzeszów, Poland, Jun. 2019, pp. 81-86.

DOI: 10.23919/MIXDES.2019.8787095

- F. Horst, A. Farokhnejad, Q.-T. Zhao, B. Iñíguez and A. Kloes, "2-D Physics-Based Compact DC Modeling of Double-Gate Tunnel-FETs," in IEEE Transactions on Electron Devices, vol. 66, no. 1, pp. 132-138, Jan. 2019.

DOI: 10.1109/TED.2018.2856891, Impact Factor: 2.704

- F. Horst, A. Farokhnejad, G. Darbandy, B. Iñíguez and A. Kloes, "Area Equivalent WKB Compact Modeling Approach for Tunneling Probability in Hetero-Junction TFETs Including Ambipolar Behavior," in International Journal of Microelectronics and Computer Science, vol. 9, no. 2, pp. 47-59, Dec. 2018.

URL: https://ijmcs.dmcs.pl/documents/10630/360081/IJMCS_2_2018_1.pdf

- F. Horst, A. Farokhnejad, B. Iñíguez and A. Kloes, "An Area Equivalent WKB Approach to Calculate the B2B Tunneling Probability for a Numerical Robust Implementation in TFET Compact Models," in 2018 25th International Conference "Mixed Design of Integrated Circuits and Systems" (MIXDES), Gdynia, Poland, pp. 45-50, Jun. 2018.

DOI: 10.23919/MIXDES.2018.8436770

- M. Graef, F. Hosenfeld, F. Horst, A. Farokhnejad, F. Hain, B. Iñíguez and A. Kloes, "Advanced Analytical Modeling of Double-Gate Tunnel-FETs - A Performance Evaluation," in Solid-State Electronics, vol. 141, pp. 31-39, Mar. 2018.

DOI: 10.1016/j.sse.2017.11.009, Impact Factor: 1.492

- M. Graef, F. Hosenfeld, F. Horst, A. Farokhnejad, B. Iñíguez and A. Kloes, "Capturing Performance Limiting Effects in Tunnel-FETs," in ISTE OpenScience Journal Nanoelectronic Devices, vol. 18-1, pp. 1-10, Feb. 2018.

DOI: 10.21494/ISTE.OP.2018.0220 
- F. Horst, A. Farokhnejad, M. Graef, F. Hosenfeld, G. V. Luong, C. Liu, Q.-T. Zhao, F. Lime, B. Iñíguez and A. Kloes, F. Horst, A. Farokhnejad, M. Graef, F. Hosenfeld, G. V. Luong, C. Liu, Q.-T. Zhao, F. Lime, B. Iñíguez and A. Kloes, "DC/AC Compact Modeling of TFETs for Circuit Simulation of Logic Cells Based on an Analytical PhysicsBased Framework," in 2017 Austrochip Workshop on Microelectronics (Austrochip), Linz, Austria, Oct. 2017, pp. 6-10.

DOI: 10.1109/Austrochip.2017.10

- M. Graef, F. Hain, F. Hosenfeld, F. Horst, A. Farokhnejad, B. Iñíguez and A. Kloes, "Analytical Modeling of RDF Effects on the Threshold Voltage in Short-Channel DoubleGate MOSFETs," in 2017 MIXDES - 24th International Conference "Mixed Design of Integrated Circuits and Systems," Bydgoszcz, Poland, Jun. 2017, pp. 127-131.

DOI: 10.23919/MIXDES.2017.8005168

- F. Horst, M. Graef, F. Hosenfeld, A. Farokhnejad, G.V. Luong, Q.-T. Zhao, B. Iñíguez and A. Kloes, "Static Noise Margin Analysis of $8 \mathrm{~T}$ TFET SRAM Cells Using a 2D Compact Model Adapted to Measurement Data of Fabricated TFET Devices," in 2017 Joint International EUROSOI Workshop and International Conference on Ultimate Integration on Silicon (EUROSOI-ULIS), Athens, Greece, Apr. 2017, pp. 39-42.

DOI: 10.1109/ULIS.2017.7962595

- F. Horst, M. Graef, F. Hosenfeld, A. Farokhnejad, F. Hain, G.V. Luong, Q.-T. Zhao, B. Iñíguez and A. Kloes, "Implementation of a DC Compact Model for Double-Gate TunnelFET Based on 2D Calculations and Application in Circuit Simulation," in 2016 46th European Solid-State Device Research Conference (ESSDERC), Lausanne, Switzerland, Sep. 2016, pp. 456-459.

DOI: 10.1109/ESSDERC.2016.7599684

- F. Hosenfeld, F. Horst, M. Graef, A. Farokhnejad, A. Kloes, B. Iñíguez and F. Lime, "Rapid NEGF-Based Calculation of Ballistic Current in Ultra-Short DG MOSFETs for Circuit Simulation," in International Journal of Microelectronics and Computer Science, vol. 7, no. 2, pp. 65-77, 2016.

URL: https://ijmcs.dmcs.pl/documents/10630/212938/IJMCS_2_2016_5.pdf

- M. Graef, F. Hain, F. Hosenfeld, F. Horst, A. Farokhnejad, B. Iñíguez and A. Kloes, "Comparative Numerical Analysis and Analytical RDF-Modeling of MOSFETs and DG Tunnel-FETs," in 2016 MIXDES - 23rd International Conference Mixed Design of Integrated Circuits and Systems, Lodz, Poland, Jun. 2016, pp. 47-51.

DOI: 10.1109/MIXDES.2016.7529698 
- M. Graef, F. Hain, F. Hosenfeld, F. Horst, A. Farokhnejad, B. Iñíguez and A. Kloes, "Numerical Analysis and Analytical Modeling of RDF in DG Tunnel-FETs," in 2016 Joint International EUROSOI Workshop and International Conference on Ultimate Integration on Silicon (EUROSOI-ULIS), Vienna, Austria, Jan. 2016, pp. 64-67.

DOI: 10.1109/ULIS.2016.7440053 


\section{UNIVERSITAT ROVIRA I VIRGILI}

COMPACT MODELING OF INTRINSIC CAPACITANCES IN DOUBLE-GATE TUNNEL-FETS

Atieh Farokhnejad 


\section{List of Symbols}

\begin{tabular}{|c|c|c|}
\hline Symbol & Description & Unit \\
\hline$A$ & Complex constant of the $1 \mathrm{D}$ wavefunction & {$[1 / \sqrt{\mathrm{cm}}]$} \\
\hline$B$ & Complex constant of the $1 \mathrm{D}$ wavefunction & {$[1 / \sqrt{\mathrm{cm}}]$} \\
\hline$C$ & Complex constant of the $1 \mathrm{D}$ wavefunction & {$[1 / \sqrt{\mathrm{cm}}]$} \\
\hline$C_{\mathrm{gd}}$ & Gate-drain capacitance & {$[\mathrm{F}]$} \\
\hline$C_{\mathrm{gg}}$ & Gate-gate capacitance & {$[\mathrm{F}]$} \\
\hline$C_{\mathrm{gs}}$ & Gate-source capacitance & {$[\mathrm{F}]$} \\
\hline$C_{\mathrm{dg}}$ & Drain-gate capacitance & {$[\mathrm{F}]$} \\
\hline$C_{\mathrm{dd}}$ & Drain self capacitance & {$[\mathrm{F}]$} \\
\hline$C_{\mathrm{sg}}$ & Source-gate capacitance & {$[\mathrm{F}]$} \\
\hline$C_{\mathrm{ss}}$ & Source self capacitance & {$[\mathrm{F}]$} \\
\hline$C_{\mathrm{BS}}$ & Bulk source capacitance & {$[\mathrm{F}]$} \\
\hline$C_{\mathrm{BD}}$ & Bulk drain capacitance & {$[\mathrm{F}]$} \\
\hline$C_{\mathrm{BG}}$ & Bulk gate capacitance & {$[\mathrm{F}]$} \\
\hline$C_{\mathrm{dep}}^{\prime}$ & Depletion capacitance per unit area & {$\left[\mathrm{F} / \mathrm{cm}^{2}\right]$} \\
\hline$C_{\text {eff }}^{\prime}$ & Effective gate capacitance per unit area & {$\left[\mathrm{F} / \mathrm{cm}^{2}\right]$} \\
\hline$C_{\mathrm{ox}}^{\prime}$ & Oxide capacitance per unit area & {$\left[\mathrm{F} / \mathrm{cm}^{2}\right]$} \\
\hline$D$ & Complex constant of the $1 \mathrm{D}$ wavefunction & {$[1 / \sqrt{\mathrm{cm}}]$} \\
\hline$D$ & Diameter of the NW TFET & {$[\mathrm{nm}]$} \\
\hline$E$ & Energy level & {$[\mathrm{eV}]$} \\
\hline$\vec{E}$ & Electric field vector & {$[\mathrm{V} / \mathrm{cm}]$} \\
\hline$E_{\mathrm{c}}$ & Conduction band energy & {$[\mathrm{eV}]$} \\
\hline$E_{\mathrm{f}}$ & Fermi energy level & {$[\mathrm{eV}]$} \\
\hline$E_{\text {f-init }}$ & Initial Fermi energy level & {$[\mathrm{eV}]$} \\
\hline$E_{\mathrm{g}}$ & Band gap energy & {$[\mathrm{eV}]$} \\
\hline$E_{\mathrm{v}}$ & Valance band energy & {$[\mathrm{eV}]$} \\
\hline$F$ & Complex constant of the $1 \mathrm{D}$ wavefunction & {$[1 / \sqrt{\mathrm{cm}}]$} \\
\hline$f$ & Fermi-Dirac distribution & {$[-]$} \\
\hline$g_{\mathrm{d}}$ & Schottky barrier small signal conductance at the drain side & {$[\mathrm{S}]$} \\
\hline
\end{tabular}


$g_{\mathrm{s}} \quad$ Schottky barrier small signal conductance at the source side $\quad$ S

$g_{\mathrm{ds}}$

$g_{\mathrm{m}}$

$\hbar$

$I_{\text {avg }}$

$I_{\mathrm{ds}}$

$I_{\text {on }}$

$I_{\text {out }}$

$i_{\mathrm{d}}$

$i_{\mathrm{g}}$

$i_{\mathrm{s}}$

$k$

$k_{1,2,3}$

$k_{\mathrm{B}}$

$l_{\mathrm{ch}}$

$l_{\mathrm{sd}}$

$L_{\mathrm{b}}$

$L_{\mathrm{G}}$

$L_{\mathrm{GD}}$

$L_{\text {GS }}$

$L_{\text {ch }}$

$\vec{n}$

$n_{\mathrm{i}}$

$N_{\text {c }}$

$N_{\text {ch }}$

$N_{\text {d }}$

$N_{\mathrm{s}}$

$N_{\mathrm{t}}$

$N_{\text {inv }}$

$m^{*}$

$m_{0}$

$m_{\mathrm{n}}$

$m_{\mathrm{p}}$

$P_{\text {avg }}$

$q$

$Q_{\text {ambi }}$

$Q_{\text {on }}$

$Q_{\text {ch }}^{\prime}$

$Q_{\mathrm{i}}^{\prime}$
Output conductance

[S]

[S]

[Js]

[A]

[A]

[A]

[A]

[A]

[A]

[A]

$\left[\mathrm{cm}^{-1}\right]$

$\left[\mathrm{cm}^{-1}\right]$

$[\mathrm{J} / \mathrm{K}]$

[nm]

[nm]

[nm]

[nm]

[nm]

[nm]

[nm]

[-]

$\left[\mathrm{cm}^{-3}\right]$

$\left[\mathrm{cm}^{-3}\right]$

$\left[\mathrm{cm}^{-3}\right]$

$\left[\mathrm{cm}^{-3}\right]$

$\left[\mathrm{cm}^{-3}\right]$

$\left[\mathrm{cm}^{-2}\right]$

$\left[\mathrm{C} / \mathrm{cm}^{-3}\right]$

$[\mathrm{kg}]$

[kg]

[kg]

[kg]

[W]

[As]

[C]

[C]

$\left[\mathrm{C} / \mathrm{cm}^{2}\right]$

$\left[\mathrm{C} / \mathrm{cm}^{2}\right]$ 
$Q_{\mathrm{i}, 0}^{\prime} \quad$ Minimum value of the channel charge per unit area

$\left[\mathrm{C} / \mathrm{cm}^{2}\right]$

$Q_{\mathrm{b}, \mathrm{d}}^{\prime}$

Charge at the tunneling barrier on the drain side

$\left[\mathrm{C} / \mathrm{cm}^{2}\right]$

$Q_{\mathrm{b}, \mathrm{s}}^{\prime}$

Charge at the tunneling barrier on the source side

$\left[\mathrm{C} / \mathrm{cm}^{2}\right]$

$Q_{\mathrm{j}, \mathrm{d}}^{\prime}$

Charge at the drain junction

$\left[\mathrm{C} / \mathrm{cm}^{2}\right]$

$Q_{\mathrm{j}, \mathrm{s}}^{\prime}$

Charge at the source junction

$\left[\mathrm{C} / \mathrm{cm}^{2}\right]$

$Q_{\mathrm{m}}^{\prime}$

1D mobile charge per unit area

$\left[\mathrm{C} / \mathrm{cm}^{2}\right]$

$R_{\mathrm{ch}}$

Channel resistance

$[\Omega]$

$R_{\mathrm{d}}$

Parasitic drain resistance

$[\Omega]$

$R_{\mathrm{S}}$

Parasitic source resistance

$[\Omega]$

$S \quad$ Subthreshold swing

$S_{\text {ideal }} \quad$ Ideal subthreshold swing

$[\mathrm{mV} / \mathrm{dec}]$

$T$

Temperature

$[\mathrm{mV} / \mathrm{dec}]$

$T_{\mathrm{HP}} \quad$ Oscillation period of the ring-oscillator in HP mode

$[\mathrm{K}]$

$T_{\mathrm{LP}}$

Oscillation period of the ring-oscillator in LP mode

$[\mathrm{s}]$

$[\mathrm{s}]$

$T_{1 \mathrm{t}}$

Oscillation period of the ring-oscillator for lt TFET

$[\mathrm{s}]$

$T_{\mathrm{pt}}$

Oscillation period of the ring-oscillator for pt TFET

$[\mathrm{s}]$

$T_{\text {tun }}$

Tunneling probability

$[-]$

Oxide thickness in NW TFET

$[\mathrm{nm}]$

TOX

Channel thickness

$[\mathrm{nm}]$

Thickness of the high- $\kappa$ oxide

$[\mathrm{nm}]$

$t_{\mathrm{hk}}$

Energy barrier shape as a function of $\mathrm{x}$

$[\mathrm{J}]$

$U(x)$

Height of the constant barrier

$V_{\mathrm{d}}$

Drain voltage

[V]

Gate voltage

$[\mathrm{V}]$

$V_{\mathrm{s}}$

Source voltage

$[\mathrm{V}]$

Drain-to-source voltage

[V]

$V_{\mathrm{gs}}$

Gate-to-source voltage

$[\mathrm{V}]$

$V_{\mathrm{DD}}$

Supply voltage of the inverter

$[\mathrm{V}]$

$V_{\mathrm{th}}$

Thermal voltage

$[\mathrm{V}]$

$V_{\mathrm{TH}}$

Subthreshold voltage

$[\mathrm{V}]$

Output voltage

$[\mathrm{V}]$

$V_{\text {in }}$

Input voltage

$[\mathrm{V}]$

Flat-band voltage

$[\mathrm{V}]$

$V_{0}$

Gate bias in the subthreshold regime

$[\mathrm{V}]$

W

Lambert's W function

$[-]$

$w_{\mathrm{ch}}$

Channel width of TFET

[nm]

$x$

Cartesian coordinate

$[\mathrm{cm}]$

$X_{\mathrm{b}, \mathrm{d}}$

Position of the tunneling barrier on the drain side

$[\mathrm{nm}]$

$X_{\mathrm{b}, \mathrm{s}}$

Position of the tunneling barrier on the source side

$[\mathrm{nm}]$ 


\begin{tabular}{|c|c|c|}
\hline$y$ & Cartesian coordinate & {$[\mathrm{cm}]$} \\
\hline$z$ & Cartesian coordinate & {$[\mathrm{cm}]$} \\
\hline$\alpha$ & Slope degradation factor & {$[-]$} \\
\hline$\Delta$ & Laplace operator & {$[-]$} \\
\hline$\Delta V$ & Channel voltage drop & {$[\mathrm{V}]$} \\
\hline$\varepsilon$ & Permittivity & {$[\mathrm{As} / \mathrm{Vcm}]$} \\
\hline$\varepsilon_{1,2,3}$ & Energy bands of an atom & {$[\mathrm{eV}]$} \\
\hline$\Theta(x)$ & Amplitude of the wavefunction & {$[1 / \sqrt{\mathrm{cm}}]$} \\
\hline$\kappa$ & Dielectric constant & {$[-]$} \\
\hline$\kappa_{\mathrm{hk}}$ & Dielectric constant of high $-\kappa$ material & {$[-]$} \\
\hline$\lambda$ & Wavelength & {$[\mathrm{cm}]$} \\
\hline$\mu_{\mathrm{n}}$ & Electron mobility & {$\left[\mathrm{cm}^{2} / \mathrm{Vs}\right]$} \\
\hline$\mu_{\mathrm{p}}$ & Hole mobility & {$\left[\mathrm{cm}^{2} / \mathrm{Vs}\right]$} \\
\hline$\mu_{\text {tun }, \mathrm{n}}$ & Tunneling mobility for electrons & {$\left[\mathrm{cm}^{2} / \mathrm{Vs}\right]$} \\
\hline$\mu_{\text {tun }, \mathrm{p}}$ & Tunneling mobility for holes & {$\left[\mathrm{cm}^{2} / \mathrm{Vs}\right]$} \\
\hline$\rho$ & Charge density & {$\left[\mathrm{C} / \mathrm{cm}^{3}\right]$} \\
\hline$\tau$ & Intrinsic inverter delay & {$[\mathrm{s}]$} \\
\hline$\Phi$ & Electrostatic potential & {$[\mathrm{V}]$} \\
\hline$\Phi_{1 \mathrm{D}}$ & Average value of the potential profile from gate to gate & {$[\mathrm{V}]$} \\
\hline$\Phi_{\mathrm{s}}$ & Surface potential & {$[\mathrm{V}]$} \\
\hline$\Phi_{\mathrm{g}}$ & Gate potential & {$[\mathrm{V}]$} \\
\hline$\phi$ & Phase function of the wavefunction & {$[-]$} \\
\hline$\Psi$ & Wavefunction & {$[1 / \sqrt{\mathrm{cm}}]$} \\
\hline$\Psi_{\mathrm{I}, \mathrm{II}, \mathrm{III}}$ & Wavefunction in associated region & {$[1 / \sqrt{\mathrm{cm}}]$} \\
\hline$\Psi_{\text {in }}$ & Incoming wavefunction & {$[1 / \sqrt{\mathrm{cm}}]$} \\
\hline$\Psi_{\mathrm{r}}$ & Reflected wavefunction & {$[1 / \sqrt{\mathrm{cm}}]$} \\
\hline$\Psi_{\mathrm{t}}$ & Transmitted wavefunction & {$[1 / \sqrt{\mathrm{cm}}]$} \\
\hline$\nabla$ & Nabla operator & {$[-]$} \\
\hline$\partial$ & Partial differential operator & {$[-]$} \\
\hline
\end{tabular}




\section{List of Acronyms}

\begin{tabular}{ll} 
Symbol & Description \\
\hline $1 \mathrm{D}$ & One-dimensional \\
$2 \mathrm{D}$ & Two-dimensional \\
$3 \mathrm{D}$ & Three-dimensional \\
$\mathrm{ABC}$ & Atanasoff-Berry computer \\
$\mathrm{AC}$ & Alternating current \\
$\mathrm{Al}_{\mathrm{x}} \mathrm{Ga}_{1-\mathrm{x}}$ As & Aluminum gallium arsenide \\
$\mathrm{As}$ & Arsenic \\
$\mathrm{B}$ & Boron \\
$\mathrm{BNG}$ & Band gap narrowing \\
$\mathrm{B} 2 \mathrm{~B}$ & Band-to-band \\
$\mathrm{CMOS}$ & Complementary metal-oxide-semiconductor \\
$\mathrm{Ch}$ & Channel \\
$\mathrm{D}$ & Drain \\
$\mathrm{DC}$ & Direct current \\
$\mathrm{DG}$ & Double-gate \\
$\mathrm{DIBL}$ & Drain-induced barrier-height lowering \\
$\mathrm{EDVAC}$ & Electronic discrete variable automatic computer \\
$\mathrm{ENIAC}$ & Electronic numerical integrator and computer \\
$\mathrm{EOT}$ & Equivalent oxide thickness \\
$\mathrm{F}-\mathrm{N}$ & Fowler-Nordheim \\
$\mathrm{G}$ & Gate \\
$\mathrm{Ge}$ & Germanium \\
$\mathrm{GaAs}$ & Gallium arsenide \\
$\mathrm{HfO} 2$ & Hafnium oxide \\
$\mathrm{HP}$ & Integrated circuit \\
$\mathrm{IC}$ & Low power \\
$\mathrm{ITRS}$ & \\
LP & \\
&
\end{tabular}




$\begin{array}{ll}\text { lt } & \text { Line tunneling } \\ \text { MOSFET } & \text { Metal-oxide-semiconductor field-effect transistor } \\ \mathrm{NW} & \text { Nanowire } \\ \mathrm{P} & \text { Phosphorus } \\ \mathrm{pt} & \text { Point tunneling } \\ \mathrm{S} & \text { Source } \\ \mathrm{SB} & \text { Single-band } \\ \mathrm{SCE} & \text { Short channel effect } \\ \mathrm{SG} & \text { Single gate } \\ \mathrm{Si} & \text { Silicon } \\ \mathrm{SiO}{ }_{2} & \text { Silicon dioxide } \\ \mathrm{SOI} & \text { Silicon-on-insulator } \\ \mathrm{TAT} & \text { Trap-assisted-tunneling } \\ \mathrm{TCAD} & \text { Technology computer-aided design } \\ \mathrm{TFET} & \text { Tunnel-field-effect transistor } \\ \text { WKB } & \text { Wentzel-Kramers-Brillouin }\end{array}$




\section{CHAPTER 1}

\section{Introduction}

\subsection{Why TFET?}

Our insatiable desire for advancement and heightening is indubitably the mover and shaker of science and is perfectly mirrored in the industry. The gigantic computers have been shrunk a thousandfold and turned to be appreciably more efficient. The fulfillment of this ever-increasing wish for better, thinner, faster and cheaper technology has only become possible because of the miniaturization of metal-oxide-semiconductor field-effect transistors (MOSFETs). This astonishing trend has brought the gadgets to a level of complexity and capability that probably few could merely imagine, a century ago. Miniaturization not only yields a reduction in device dimensions, but also improves power efficiency.

At the beginning, according to the scaling rules, the aim was to shrink the vertical and horizontal dimensions of the MOSFET by a specific scaling factor and increasing the doping concentration by the similar factor in a manner such that the electric field inside the device remains unchanged in relation to the original structure [1-3]. In this way the down scaling of MOSFETs has endured for over 30 years, from the micrometer to the nanometer regime [4]. However, this trend began to reach its physical limits when the $65 \mathrm{~nm}$ node was reached in 2000 [4]. The electrostatic control of the gate over the channel was negatively impacted by down scaling due to the high electric field. Hence, from this point on, shrinking the structure of the MOSFET was not any further possible just by scaling its dimensions [4]. The leakage current, the subthreshold swing degradation and other short-channel effects (SCEs) are the important challenges for the further down scaling of the MOSFETs.

In the subthreshold or weak inversion region of the MOSFET, where the gate voltage $\left(V_{\mathrm{gs}}\right)$ is lower than the subthreshold voltage $\left(V_{\mathrm{TH}}\right)$ and the inversion channel is not fully formed, the charge carriers may anyway flow from the source to the drain. Considering the band diagram of a MOSFET illustrated in Fig. 1.1, the higher the gate voltage, the lower the potential barrier would be. As a result, more electrons can move from source to the drain. This current flow is known as the subthreshold leakage current in MOSFET [5]. By reducing the size of the 
MOSFET, the gate oxide which is made of Silicon dioxide $\left(\mathrm{SiO}_{2}\right)$ in addition, gets thinner and leads to higher leakage current through the gate terminal [6].

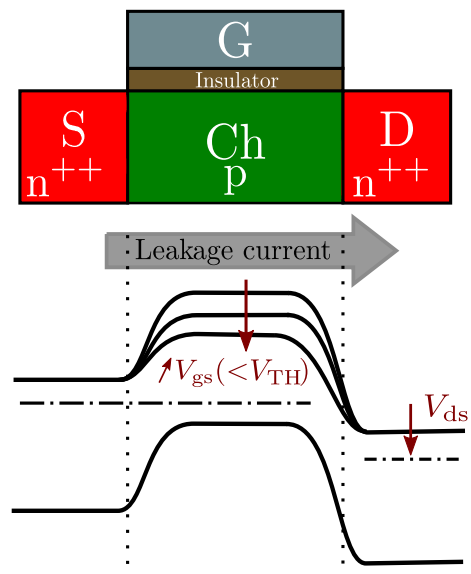

Figure 1.1: Schematic of an $n-$ channel MOSFET and its band diagram. Increasing the gate voltage in subthreshold regime $\left(V_{\mathrm{gs}}<V_{\mathrm{TH}}\right)$ results in the potential barrier lowering and consequently increasing the leakage current from source to the drain [5].

The subthreshold slope of a MOSFET refers to the required change in the gate-source voltage in order to increase the drain current $\left(I_{\mathrm{ds}}\right)$ by a factor of 10 . The reciprocal value of this ratio is called subthreshold swing $(S)$ and is given as:

$$
S=\frac{d V_{\mathrm{gs}}}{d \log _{10} I_{\mathrm{ds}}} .
$$

Although according to this definition the subthreshold slope is the inverse of $\mathrm{S}$, in the literature this value is often quoted as subthreshold slope $[7,8]$.

Due to the thermionic-emission based current transport mechanism of MOSFETs, their minimum subthreshold swing at the room temperature is theoretically limited to $60 \mathrm{mV} / \mathrm{dec}$ $[5,9]$. At a constant $S$ the reduction of the supply voltage leads to a higher leakage current and a worse ratio of $I_{\mathrm{on}} / I_{\mathrm{off}}$.

By reducing the dimensions of the MOSFET, the widths of the depletion layers on the source and drain side become comparable to the channel length of the device. In this case, the potential of the channel is influenced by a strong lateral and also transverse electric field [1]. The effects resulting from this two-dimensional distribution of the channel potential and the strong electric field are described as short-channel effects. The drain-induced barrier-height lowering (DIBL), threshold voltage roll-off, off-state leakage and subthreshold swing degradation are some examples among these SCEs [10].

The innovative methods and technologies have made it possible to tackle these challenges and go even further with scaling. For instance, by using high- $\kappa$ insulators instead of $\mathrm{SiO}_{2}$ and taking advantage of strained silicon technology the leakage current has been reduced [6]. 
These alternative materials allow the same dielectric capacitance to be achieved with a higher dielectric thickness compared to $\mathrm{SiO}_{2}$. So, the tunneling barrier would be thicker and can block the gate leakage [11]. Furthermore, multiple-gate structures by increasing the electrostatic control of the gate over the channel make it possible to a better grip on SCEs [12, 13].

Considering the fact that further scaling of the MOSFET leads to other undesirable problems such as quantum mechanical effects, together with the $60 \mathrm{mV} / \mathrm{dec}$ limit of the MOSFET and furthermore the strong interest to reduce the subthreshold swing and suppress the power consumption of the circuits, the option to investigate new structures remains. Hence, alternative devices with abrupt switching behavior, which is also not affected by supply voltage, need to be sought $[7,9,14]$.

Among these devices Tunnel field-effect transistors (TFETs) have been studied as viable successor of conventional MOSFETs. They can overcome the $60 \mathrm{mV} / \mathrm{dec} S$ which is due to their alternative current transport mechanism, the so called band-to-band (B2B) tunneling. It helps TFETs to operate at a low supply voltage and therefore have a lower power consumption. TFETs show a lower leakage current and in comparison to MOSFETs they have a greater level of stability against short-channel effects $[7,14,15]$. Moreover, they are compatible with the complementary metal-oxide-semiconductor (CMOS) fabrication process.

In 2004 for the first time Wang has presented TFETs as the potential successor of MOSFET for low power circuits [16]. In the same year Appenzeller presented a three-terminal tunneling device using a carbon nanotube as the channel with a subthreshold swing of $40 \mathrm{mV} / \mathrm{dec}$ [17]. This has led to more attention being paid to TFETs, and then several research groups began to investigate these transistors.

The drawback of TFETs is their low on-current. It is challenging to reach a high on-current without also increasing the off-current and yet keeping the subthreshold swing smaller than $60 \mathrm{mV} / \mathrm{dec}$ [18]. Above all, the compatibility to the CMOS technology should also be considered. A combination of different technologies is therefore required to meet this challenge.

Some possibilities to tackle this challenge and enhance the current are using III-V semiconductors, implementing straining techniques, switching to 2D materials and taking advantage of line tunneling [18-20]. As an example, in 2011 Dewey presented a III-V TFET that by taking advantage of hetero-junction achieved a steep subthreshold swing $(S<60 \mathrm{mV} / \mathrm{dec})$ and high on-current [21]. In 2013 Knoll reported that the strained Si nanowire (NW) complementary TFET, at room temperature and drain voltage of $0.5 \mathrm{~V}$, shows a minimum subthreshold swing of $30 \mathrm{mV} / \mathrm{dec}$ and an on-current $>10 \mu \mathrm{A} / \mu \mathrm{m}$ [22]. In 2017 Memisevic demonstrated an InAs/InGaAsSb/GaSb vertical TFET NW which at the drain voltage of $0.3 \mathrm{~V}$ exhibits a minimum subthreshold swing of $43 \mathrm{mV} / \mathrm{dec}$ and on-current of $10 \mu \mathrm{A} / \mu \mathrm{m}$ [23].

\subsection{Necessity of Device Simulation and Compact Model}

Considering the complexity and high fabrication expenses of new structures such as TFETs, the first step for its design and production is the process and device simulation. It helps 
to improve the processing technologies, describe the behavior of the device and reduce the time and resources on test wafers. For this purpose there are powerful tools existing such as Technology Computer-Aided Design (TCAD) softwares provided by companies such as Synopsys [24], Silvaco [25], Global TCAD solutions [26], Crosslight [27] and Cogenda Software [28].

Such a program provides finite-element simulation. Once all the physical parameters of the structure such as dimensions, materials and doping profile are defined, the structure is meshed. That is to say, the structure is divided into many small geometric elements and represented in form of a finite-element structure. Afterwards, depending on the activated models, TCAD solves the physical partial differential equations for every single grid point in the structure. The TCAD simulations usually require a great deal of time and are very computationally demanding. Therefore, in order to enable the circuit simulation including millions of TFETs, compact models of circuit elements are required. Compact models have to be simple and fast, but also accurate in order to provide circuit designers with suitable results. In fact, this is essentially the point that makes the compact modeling field particularly challenging [29].

Principally for circuit analyses three types of models including DC, AC and transient are needed. A DC model predicts the device current in the case that the voltages are fixed and do not vary with time and ignores the energy-storing behavior of the semiconductor device. In an $\mathrm{AC}$ model, it is considered that the input voltage changes very slowly with time. It is a small-signal model which calculates the current variations by means of linear relations and includes the effect of energy-storage elements in the structure of the device [1]. A transient model is a large-signal dynamic model which calculates the current for the case that the input voltage varies with time [1]. In order to provide the mean to do the transient analysis, compact models for the capacitances and their charging behavior in TFETs are required. In fact the switching speed of an integrated circuit (IC) is depending on the capacitances. Hence, the focus of this thesis lays on compact modeling of intrinsic capacitances in this type of transistors.

Considering the importance of TFETs as a potential successor of MOSFETs technology, a lot of investigations have been carried out on the physics-based compact modeling of TFETs. Mostly these works are focused on the potential characteristic and current in TFETs [30-35]. Nevertheless, also some models to estimate the terminal capacitances are represented. For instance, Yang et al. in [36] have presented a compact model for TFET capacitances using BSIM3 equations [37]. Lu et al. in [38] and Zhang et al. in [39, 40] have developed an analytical charge model for Si double-gate (DG) TFETs based on a surface potential model. Lu et al. in [41] have calculated the terminal charges in TFET using a parameterized empirical expression. Based on this, the capacities are derived by numerical differentiation. The fact that some of these models use numerical iterative methods and that they are very complicated makes them somewhat inadequate and less effective for the use in SPICE and circuit simulations. The charge-based model introduced in this work is very simple and flexible and contains no iterative process, which makes it SPICE friendly and suitable for circuit simulations. In addition, unlike most of the reported models, this compact model is validated not only by TCAD simulations 
of a single device, but also by measurement data of fabricated TFETs with various structures.

\subsection{Outline of the Thesis}

Chapter 2 is a brief history of transistors and highlights some of the important inventions that have brought technology way beyond expectations and fantasies. This chapter shows how the need for better and faster computers in combination a perfectionist competitive spirit leads to such a great progress and achievements.

Chapter 3 is about the theoretical physics and mathematics backgrounds which are essential to understand this work. The characteristics of semiconductor materials in terms of atomic construction and band structure, doping, quantum mechanical effect of tunneling and the methods to calculate the tunneling probability are introduced in this chapter.

Based on the theories, in chapter 4 the working principle of TFETs is explained. By giving consideration to the device geometry and band diagram, different states and operation regions of transistors are described. Then the current-voltage and the capacitance-voltage relations of TFETs are introduced and explained. This part also shows the capacitances in the TFET, on which the compact model focuses. Furthermore, the capacitances of TFET and MOSFET are briefly compared in the end of the section.

Chapter 5 starts with the existing charge model which is used to develop the intrinsic capacitance model. The steps which are taken to adjust the charge equation to the TFET structure are explained. Afterwards, it is shown how the capacitances in the TFET are calculated by the model. Moreover, it is presented how the effect of parasitic elements as well as the Schottky barrier effect at the terminals are mathematically implemented in the model.

The validity of the capacitance model is investigated in chapter 6 . The capacitance model is firstly compared to the results of the TCAD simulations. In the next step it is verified using the measurement data of a fabricated p-type planar TFET. The deviation from measurements have shed light on the importance and effect of parasitic elements on the capacitances in TFET. Hence, in this part a theory about the impact of the Schottky barrier contacts of capacitances is introduced. This theory is then examined on TCAD simulation results of an n-type TFET to see if they produce the same pattern as in the measurements. Further verification of the model is carried out by means of measurement data of a fabricated n-type NW.

After model verification, the intrinsic capacitance model is combined with an existing compact DC model [35] and implemented in Verilog-A language. In order to examine the robustness of the compact model, TFET-based inverters and ring-oscillators are simulated in in chapter 7 . These simulations are executed for different device parameters and also for the case that the on-current of the TFET by taking advantage of line-tunneling is improved.

Chapter 8 presents a summary of the entire thesis, highlights the results which are obtained in the course of this dissertation and the points which are still not implemented in the model and need to be in future studies. 


\section{CHAPTER 2}

\section{History of Microelectronics}

To know where we are standing in microelectronics technology and also where the need for such an advanced improvement in this area comes from, it is helpful to glance over the path it has taken so far. All the way from giant mechanical computers to invention of tiny bipolar transistors to nanoscale devices. Hence, in this section some of the most important achievements which have changed the direction of industry and technology are briefly addressed.

\subsection{Mechanical Computers}

The concept of automatic computing machine was firstly introduced in 1822 by Charles Babbage, a mathematics Professor in Cambridge University. As Babbage and his astronomer friend, John Herschel, were reviewing various publications of mathematical tables like natural logarithm, integrals and astronomical tables, they have actually found many errors and variations. Hence, he came about the idea to make a machine which is capable of automatic production of mathematical tables infallibly. Babbage's first proposed mechanical computer, which today is called Difference Engine No. 1, was designed using finite differences methods to solve up to seventh order polynomial equations. As it can be seen in Fig. 2.1, the Difference Engine was built entirely from mechanical components such as gears, ratchets and rods instead of electronic components. To avoid possible human errors, it was planed to be connected to a printer to make hard copies of equations results [42].

The mechanical computer was massive and its reliability depended drastically on the accuracy of the fragments used in it, which in reality meant that its use was very expensive. Anyhow, Babbage always kept going ambitiously and came about so many other ideas for instance reducing the size of mechanical computer, making them programmable and using them for beyond mathematics purposes. Despite investing lots of money and work, unfortunately these notions never got completed and did not come to fruition in Babbage's lifetime. All in all it can be said that the attempts to automatic computation in 19th century did not have much success and this procedure came closer to the goal in 20th century [43]. 


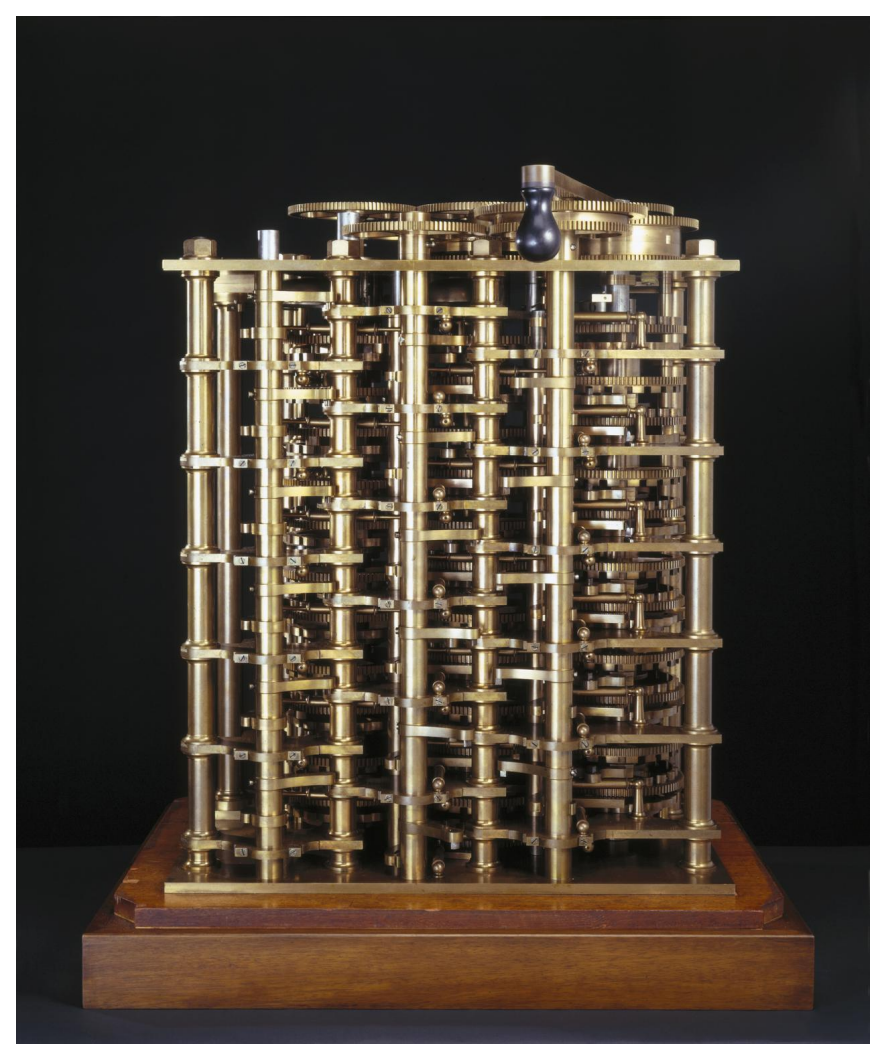

Figure 2.1: Small part of Difference Engine No.1 [44].

\subsection{Electronic Computers}

The invention of the vacuum triode ushered the way to employ binary logic functions and the possibility to replace mechanical computers with electronic computers [45].

In 1937 Professor John Vincent Atanasoff and his graduate student Clifford Berry, by using vacuum tubes built the first automatic electronic digital computer. Anyhow, neither of them had ever applied for a patent. The Atanasoff-Berry computer (ABC) could not be reprogrammed and was designed just to solve linear equations. But in any case, it was pioneer in using binary system to represent digits or data and electronic switches, instead of mechanical elements, to do the calculations. In the same era there were some other experiments with computers using vacuum tube circuits existing such as Konrad Zuse's Z3, Manchester machine, Colossus and ENIAC. Colossus was developed in 1941 for military purposes by British engineer Tommy Flowers and it was a fully functional electronic computing machine. Nevertheless, because of the secrecy nature of Colossus and to avoid exposing the secrets of its technology, it was destroyed after the war and left ENIAC to be the pioneer to step from mechanical to 
fully electronic computers [42, 45]. In 1943 J. Presper Eckert and John Mauchly began the construction of ENIAC at the University of Pennsylvania for the United State Army's research, and by 1946 it was completed.

Years later, there were discussions about that who was really the inventor of digital computers and whether ABC was really the first electronic computer. Finally, in 1973 the US federal court declared John Atanasoff as the inventor of first electronic computer and for the ease of developers the patent rights of it were dedicated to public domain [45].

Despite the vote of district court, ENIAC is still widely known as the first electronic digital computer. Similar to the mechanical computers, ENIAC was giant (see Fig. 2.2) and contained almost 18,000 vacuum tubes, occupied over $150 \mathrm{~m}^{2}$ of space and it weighed about $30 \mathrm{t}$. It was programmable, but since programes were hard coded into the machine, an implementation of them was only possible by manipulating the switches, function tables and wiring of the machine. Afterwards, the program could be executed step by step for debugging. Nevertheless, it was complicated and very time consuming to perform a complex program on the ENIAC. Besides all difficulties in programming, maintenance of those giant computers was another big problem. Vacuum tubes consume great deal of power and could get very warm. Consequently, they required a lot of space and the rooms that were occupied with vacuum tubes needed to be cooled down continuously, which meant even more energy consumption. Furthermore, considering the short lifetime of vacuum tubes, they had to be replaced quite often. Overcoming these problems has been made possible only by the invention of transistors [42, 45].

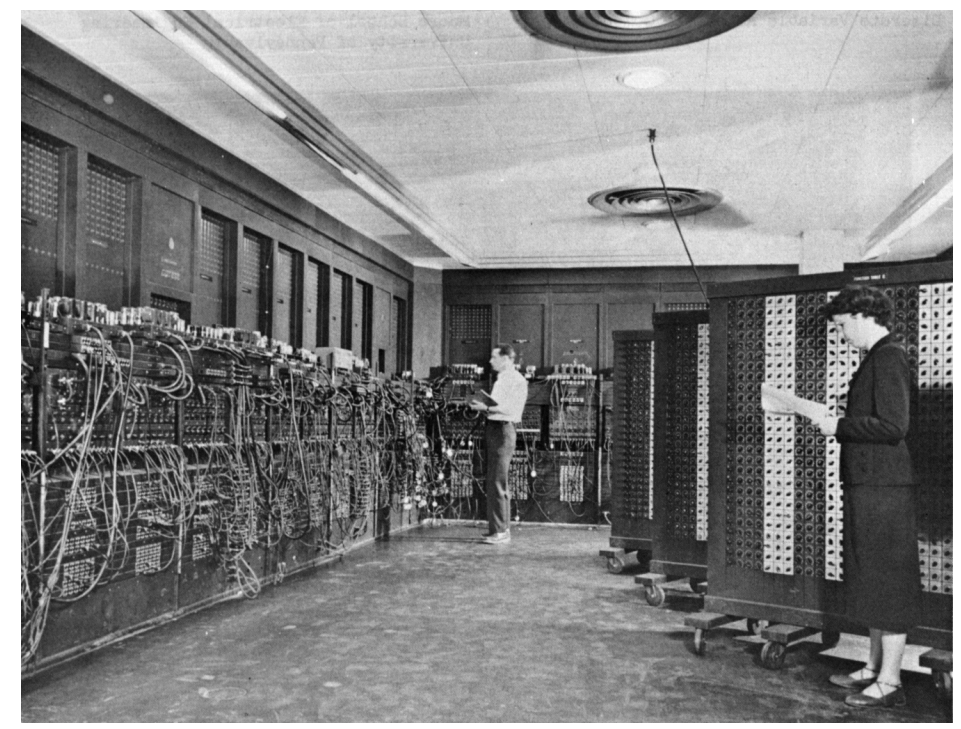

Figure 2.2: ENIAC was massive and its maintenance was complicated, time consuming and energy demanding [46]. 


\subsection{Invention of Transistors}

The biggest challenge in twenties was to find a replacement for vacuum triode and the question was how to go further with electronic industry. Mervin Kelly, the Bell Labs' Director of Research, could see from very beginning of his career the importance and potential of a triode based on semiconductor material to make a big change. To start, he chose the young talented physicist William Shockley from MIT as the leader of this project. Additionally, Kelly gave him the freedom to work with any group working on different projects in Bell Labs to widen his horizon and overcome the obstacles. There he began his work by participating in ongoing projects and studying all work on semiconductors [45].

Soon after, in 1939 Shockley came about the idea to combine Schottky's Metal-Semiconductor Junction rectifier with Brattain's cuprous oxide semiconductor which has led to Shockley's field-effect theory. According to his theory, the electrical field close to a semiconductor sample affects the resistance of it and hence, cause the current flows through the semiconductor. Based on field-effect theory and by the help of Brattain, Shockley started some experiments to build a solid-state triode. However, Shockley and Brattain's first attempts did not succeed [45].

Over the course of time, Bell Labs team gained deeper knowledge on semiconductor materials. Also new concepts such as dopant, n-type and $\mathrm{p}$-type semiconductors were addressed by researchers. Thereafter, Walter Brattain and John Bardeen, the solid-state physicists, started to work together on Shockley's field-effect triode. Although Shockley was the leader of this project, he was not much involved with experiments they where dealing with. Finally in 1948 the first functional point contact transistor was represented (see Fig. 2.3) [45].

Shortly after that, Brattain and Bardeen were listed as transistor inventors. Shockley, who was not really happy about this news, has pulled himself together and created his junction transistor or bipolar transistor. Bipolar transistors were manufactured for several years but it was not the end of the transistor story, rather just the beginning of breathtaking changes and progresses to a new era. In 1958 as Atalla's team were studying the features of a silicon-silicon dioxide interface in Bell Labs, they observed something very similar to the field-effect theory of Shockley. They realized if a metal electrode is deposited on top the oxide, by applying voltage to this electrode, at the silicon-oxide interface a conductive channel is build. Based on this observation, in 1960 Atalla developed the MOSFET [45].

In comparison to bipolar transistors, MOSFETs were easier to be reduced in size and voltage. Furthermore, the power consumption of MOSFETs was lower. Probably at that time the significance of the miniaturization of the transistor and its affects on chip industry was still not fully clear. However, shortly after it turned to be a crucial factor in semiconductor technology. Considering all advantages of MOSFET, they easily and rapidly found their place on large-scale chips and replaced those produced using bipolar transistors [45]. 


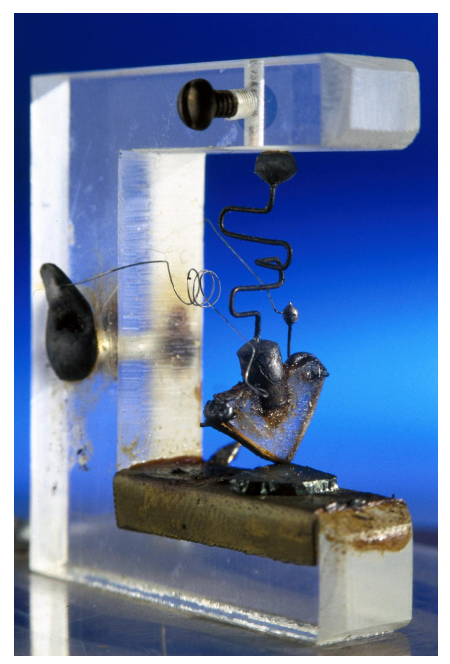

Figure 2.3: First point contact transistor represented by Brattain and Bardeen [44].

\subsection{Moore's Law}

In 1958 Jack Kilby as well as Robert Noyce came up with the idea to fabricate all required components for a circuit on one single piece of semiconductor material. Combining this idea with the use of metalized connection patterns printed on a board, known as printed circuit board technology, led to the invention of IC [45]. The combination of integrated circuit and MOSFET brought the chip industry into a completely new era. By mid-1960s the biggest challenge was to increase the features and capabilities of chips in an economically reasonable way. That is to say, squeezing more and more transistors on a chip without expanding the size and costs of the chip. Miniaturization is not only about reducing the size of transistors, but also lowering power consumption and increasing switching speed to finally achieve an even faster and more powerful computers.

In 1965 Gordon Moore, the outstanding chemist and Fairchild Semiconductor's director, published an article titled "Cramming more components onto integrated circuits" [47]. In this article the progress of microelectronics industry between 1962 to 1965 is reviewed. Moore discovered that the number of transistors on a chip is doubled every twelve months and predicted that this trend would remain constant for at least ten years (see Fig. 2.4). This prediction turned to an autogenous road map for semiconductor industry and made its name as Moore's Law. Later on he decided that the complexity is doubling every two years, however, his forecast proved to be surprisingly precise.

A simple comparison between modern computers or smart phones and their predecessors, who are not even a decade older, is enough to see how the semiconductor industry has influenced the entire world. Undoubtedly, this is the most relentless technology of all time. It is over 50 
years since the observation of Gordon Moore and Moore's Law is still valid and transistors are now scaled into nanometer regime. This question has always been existing, that how further miniaturization can go. It is obvious that in each step, not only the complexity of chips increases, but also the manufacturing progress becomes more difficult and faces greater challenges. Nonetheless, all obstacles and limitations have never been able to stop the number of transistors growing on a chip. Whenever the progress has closely reached its limit, innovation and creativity of researchers has turned it to an opportunity.

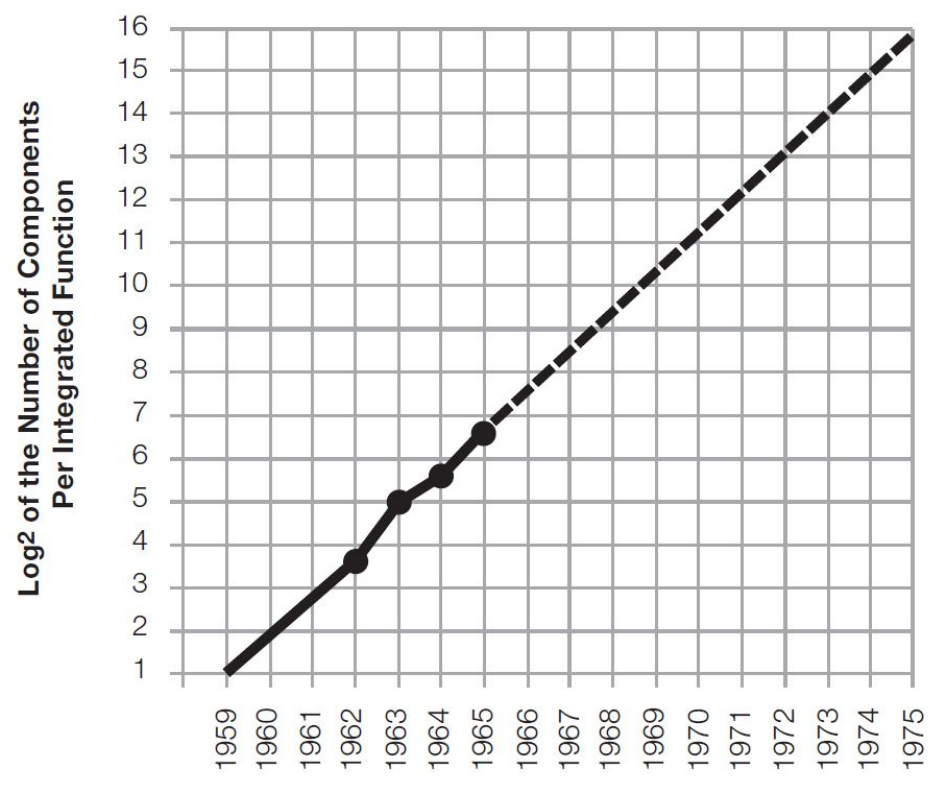

Year

Figure 2.4: Number of transistors on integrated circuit versus year according to Gordon Moore's observation in 1965. He predicted that this number doubles every year [47].

By 2019, the size of transistors has been shrunk to $7 \mathrm{~nm}$ and the transistor count has grown to tens of billions on a chip [48]. In such a tiny dimension charge carriers show a special behavior known as quantum tunneling, which is explained in detail in the Sec. 3.2. Since it is impossible to avoid this phenomenon in the nanometer regime, only using new materials, novel device structures and production techniques can actually be of help. TFETs are among recently designed transistors. They work based on quantum tunneling and show the feasibility to overcome challenges on the way of miniaturization. Further details about TFETs are discussed in chapter 4 . 


\section{CHAPTER 3}

\section{Theoretical Backgrounds}

The first step to start with modeling the behavior of the TFET is understanding the physical and mathematical principle which a TFET is based on. Sec. 3.1 focuses on the structure of semiconductor materials as single atom and also as a crystalline structure. In Sec. 3.2 the physical basis of tunneling is explained followed by the tunneling probability in Sec. 3.4. In this section, depending on the shape of the barrier, different methods to calculate the tunneling probability are considered.

\subsection{Semiconductor Materials}

Concerning the conductivity, solid state materials are divided into three categories: insulators, semiconductors and metals. In an insulator all electrons are tightly bound and therefore it can not conduct an electric current. In a metal, the valance electrons of atoms can move freely within the crystalline structure, which means higher concentration of free charge carriers and thus higher conductivity. Semiconductors, as it comes from their name, are between these two groups. Their conductivity in their natural state is poor but it can be varied by many factors such as heat, illumination, electric field and doping. This property makes semiconductors particularly suitable for the usage in the manufacture of electrical appliances. In the following sections the Fermi distribution, band structure of semiconductors and the effect of dopant on their conductance are analyzed.

\subsubsection{Fermi Distribution}

The probability that an electron occupies an available energy state $E$ is given by the Fermi-Dirac distribution function as following:

$$
f(E)=\frac{1}{1+\exp \left(\frac{E-E_{\mathrm{f}}}{k_{\mathrm{B}} T}\right)}
$$


where $k_{\mathrm{B}}$ is the Boltzmann constant and $T$ is the temperature of the material. The $E_{\mathrm{f}}$ presents the Fermi level, which refers to the energy level in which the probability to find an electron is exactly $50 \%\left(f\left(E_{\mathrm{f}}\right)=1 / 2\right)$.

Considering Fig. 3.1, at $\mathrm{T}=0 \mathrm{~K}$ the Fermi distribution shows an abrupt transition from unity to zero. That means all energy levels above $E_{\mathrm{f}}$ are empty, so $f(E)=0$ and all energy levels below $E_{\mathrm{f}}$ are full, so $f(E)=1$.

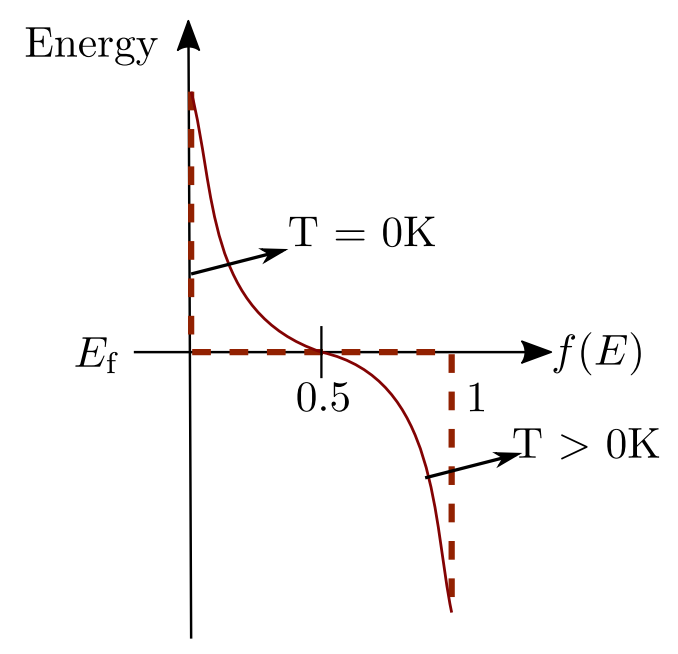

Figure 3.1: Fermi distribution at $\mathrm{T}=0$ and $\mathrm{T}>0 \mathrm{~K}[8]$.

By increasing the temperature the Fermi distribution changes and becomes smoother. As it is illustrated in Fig. 3.1, in this case it cannot be said that all energy levels above $E_{\mathrm{f}}$ are empty, it is rather likely that an energy state is occupied by an electron. The higher the temperature, the higher the probability to find an electron above the Fermi level.

Fermi level of an intrinsic semiconductor in equilibrium condition lays almost in the middle of its band gap and is referred to as intrinsic Fermi level $\left(E_{\mathrm{i}}\right)[1,8,49]$.

In the case that the considered $E$ is higher than $3 k_{\mathrm{B}} T$, the exponential function in the denominator of the Eq. (3.1) gets much bigger than one. Therefore, the carrier concentration can be approximated by Maxwell-Boltzmann statistics $[1,50]$ as following:

$$
f(E)=\exp \left(-\frac{E-E_{\mathrm{f}}}{k_{\mathrm{B}} T}\right)
$$

\subsubsection{Band structure}

According to the Pauli principle, each energy state with a certain quantum number can only be occupied with two electrons with opposite spins $[8,50]$. In other words, an atom orbital has maximum two electrons. When two or more atoms approach to form a bond, the energy levels split as it is shown in Fig. 3.2. Now considering a complex system with a large number of 
atoms in its structure, these discrete energy states of the single atoms are widened, superposed and merge into energy bands. There are bands in which the charge carriers can move freely and, by contrast, there are bands in which they are involved in covalent bonds, thus charges are immobile. The energies below the highest immobile energy state are called the valence band, while the energies above the lowest mobile energy state are called the conduction band. Due to quantum physics, there are energy ranges in which no standing waves of electrons fits. These forbidden energy states between conduction band and valence band are called the band gap [1].

Generally, the band structure of a material explains how atoms and electrons in that substance interact. Figure 3.3 shows the difference in the band structure of the three aforementioned groups of solids. It can be seen that insulators have a big band gap $\left(E_{\mathbf{g}}>5 \mathrm{eV}\right)$. Their valence band is completely filled and their conduction band is empty. In contrast to insulators, metals have no band gap and as it is illustrated, the valence band and conduction band overlap which means they have plenty of free electrons in their structures. In semiconductors the energy gap between the valence band and conduction band is adequately small. Therefore, the electrons in the valence band by absorbing enough thermal energy can be excited to the conduction band, leave behind a hole in the valence band and move freely in the lattice.

The materials which are widely used in semiconductor technology are from column III, IV and $\mathrm{V}$ of the periodic table. A semiconductor can be composed of only one element, such as Silicon ( $\mathrm{Si}$ ) and Germanium (Ge), which is called elemental semiconductor or it can be a combination of two or more elements from group III and V such as Gallium Arsenide (GaAs) or Aluminum Gallium Arsenide $\left(\mathrm{Al}_{\mathrm{x}} \mathrm{Ga}_{1-\mathrm{x}} \mathrm{As}\right)[8,51]$. Depending on different properties such as the band gap of these materials they can be used for particular purposes.
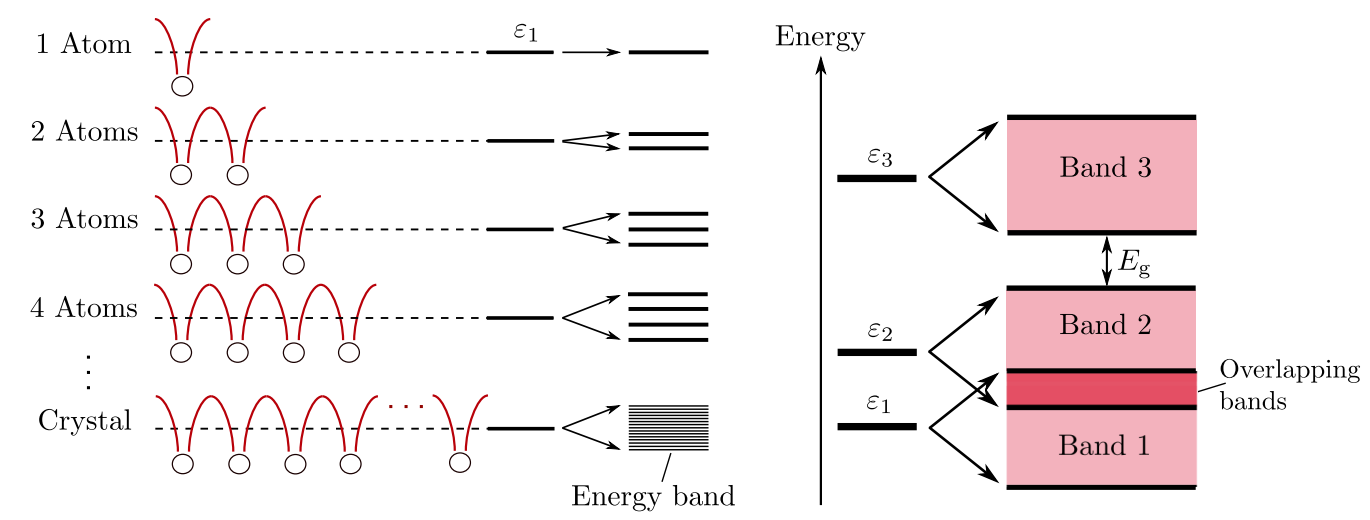

Figure 3.2: Expansion of discrete energy levels of a single atom in the vicinity of other atoms and finally into energy bands in a crystal [8]. 


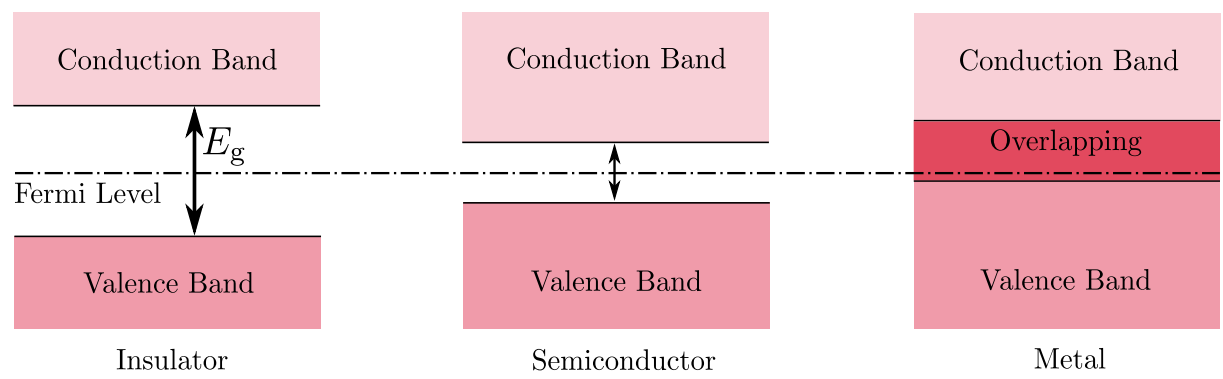

Figure 3.3: Band structure schematic of an insulator, semiconductor and a metal [8].

\subsubsection{Doped Semiconductors}

To enhance the conductivity of semiconductors, specifically some impurity atoms are added into their crystal lattice. This process is known as doping and the material is called doped or extrinsic semiconductor. By combining doped semiconductors of different types, several electrical components are fabricated such as $\mathrm{p}-\mathrm{n}$ junction which is the building block of diodes and transistors. The materials that are mostly used in technology as dopants are Boron (B), Phosphorus (P) and Arsenic (As) [1, 52].

Boron is an element in the periodic table group III with three valence electrons and silicon, the most used semiconductor in history of microelectronics, has four valance electrons. Thus, by adding a Boron atom into the silicon crystal it accepts an electron from lattice, makes a negative ion and releases an excess hole in the lattice. Dopants from main group III are called acceptors and the semiconductor doped with them is a p-type material. In this case the Fermi level is closer to the valence band of the semiconductor as it is shown in Fig. 3.4(a) $[1,8]$.

When a semiconductor is doped with impurities from group $\mathrm{V}$, which have five valence electrons, it is called n-type. These dopants add an extra electron to the semiconductor crystal, and therefore they are called donors. In an n-type material the Fermi level lays closer to the conduction band (see Fig. 3.4(b)) [1, 8].

If a semiconductor is doped with a concentration higher than $10^{19} \mathrm{~cm}^{-3}$, it is considered as heavily doped and is called degenerate. In this case, the carrier concentration is calculated with the Eq. (3.1). If the Fermi level lays within the band gap with a distance of a few $k_{\mathrm{B}} T$ from either conduction or valence band, it is called nondegenerated [1]. In such a semiconductor the carrier concentration is approximated by Eq. (3.2) [1]. 

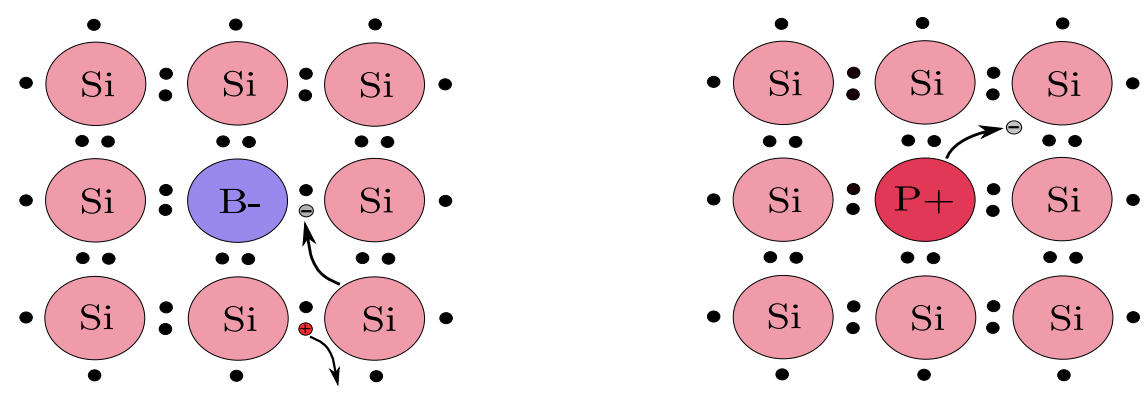

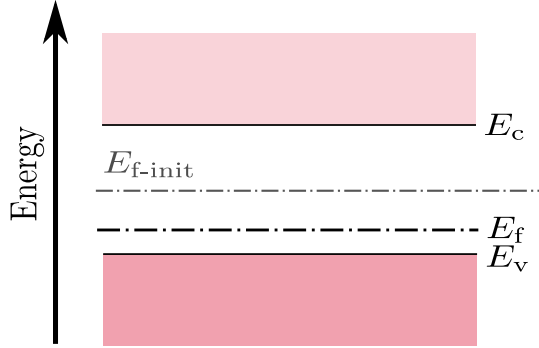

(a)

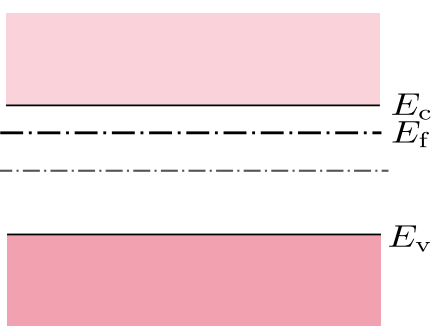

(b)

Figure 3.4: Schematic representation of a silicon crystal doped with impurity atoms and the effect of doping on its band structure. (a) By doping silicon with Boron atoms, a free hole is added to the lattice. (b) Doping silicon with Phosphorus releases a free electron to the structure. The $E_{\mathrm{f}-\text { init }}$ refers to the initial position of the Fermi level before doping.

\subsection{Quantum Tunneling}

Quantum mechanics refers to a branch of physics which describes incidences at the microscopic scale. It can explain the nature of the universe, yet it contradicts classic mechanics and the idea of how it all works. Unlike classical physics, where everything follows clear rules and is consequently predictable, quantum physics is based on probabilities. As the Heisenberg's uncertainty principle describes, quantum objects do not have a designated position before they are observed. Rather, the particles are defined with a so called wavefunction $\Psi$, which indicates the probability where a particle is most likely to be found.

According to the classical mechanics, there are only two possibilities for a charge carrier that encounters an energy barrier; either the energy of the carrier is higher than the barrier and thus it can overcome the barrier or it is not the case and the particle gets reflected. But in quantum physics another scenario is also considered, in which the charge carrier despite its low energy manages to get into and through the barrier. This phenomenon, which is inevitable in atomic and subatomic scale, is known as tunneling. Quantum tunneling is the fundament of many physical processes such as radioactivity and also operation of certain semiconductor devices including TFETs.

Figure 3.5 illustrates the incoming wavefunction $\Psi_{\text {in }}$ of a free electron. The electron flow 
encounters a constant barrier of height $U_{0}$ and thickness $L_{\mathrm{b}}$, part of it is reflected $\Psi_{\mathrm{r}}$, another part enters the barrier, its amplitude decays and then appears on the other side of the barrier as transmitted wavefunction $\Psi_{\mathrm{t}}$. The mathematical formulation that can describe the wavefunction behavior, is the Schrödinger equation. Solving this equation gives the probability to find the quantum object at a specific position. Depending on the shape of the barrier, different types of tunneling are existing and consequently, particular methods are used to solve the Schrödinger equation. In the following the categories of carrier transport mechanism, Schrödinger equation and different approaches to solve it are explained in detail.

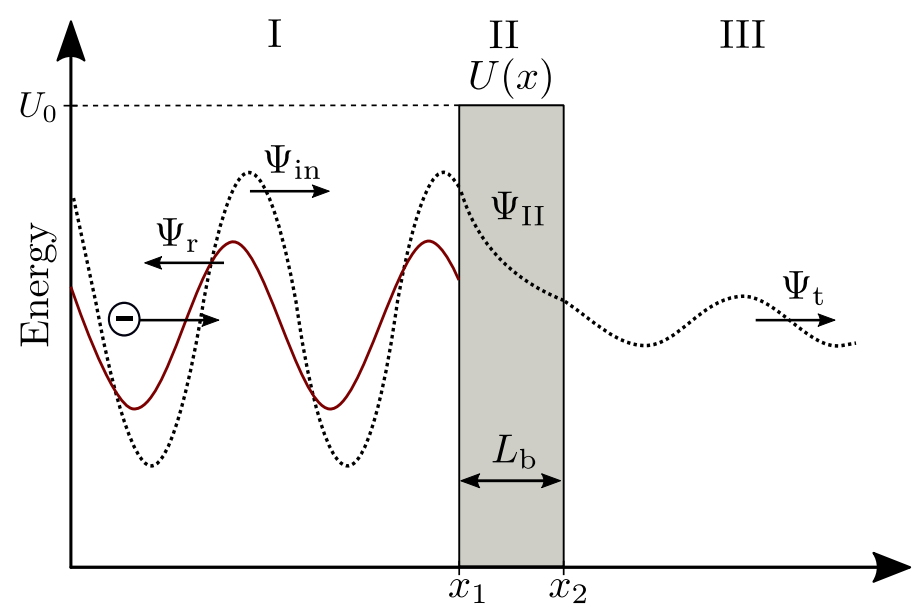

Figure 3.5: Wavefunction describes the behavior of an electron. The incoming wave encounters the rectangular shape barrier. There is the probability that part of it is reflected and part of it can tunnel through the barrier.

\subsection{Tunneling Events}

Considering the band structure there are two types of tunneling existing: single-band (SB) and B2B tunneling. Figure 3.6(a) illustrates the SB tunneling process in a SemiconductorInsulator-Semiconductor structure. It can be seen that this process is divided to three further sub-categories called: Fowler-Nordheim tunneling $(\mathrm{F}-\mathrm{N})$, direct tunneling and trap-assistedtunneling (TAT). The $\mathrm{F}-\mathrm{N}$ tunneling refers to the case that the charge carriers tunnel through the triangular barrier which is shaped due to the applied electric field. This mechanism occurs regardless of the thickness of the barrier, as long as the field effect is large enough. In direct tunneling carries penetrate through the whole barrier. Therefore, it is expected just for thinner barriers $(<5 \mathrm{~nm})[49]$.

The TAT takes place due to the existence of traps in the band gap of materials. Traps are actually defects in the structure of the material and are caused by the fabrication process. In this case, the charge carriers do not tunnel through the entire thickness of barrier. Rather, 
firstly they penetrate from one energy band to a trap inside the barrier and then either hop to the next trap or tunnel to the other side of the barrier.

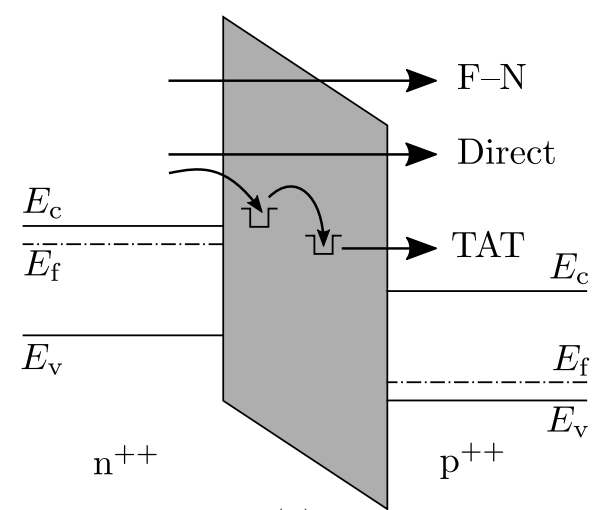

(a)

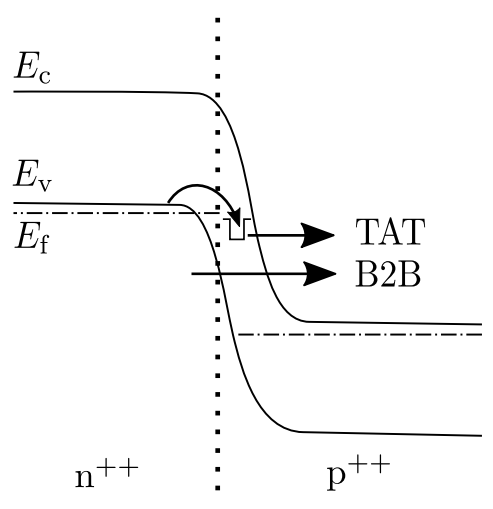

(b)

Figure 3.6: (a) Different types of SB tunneling in a semiconductor-insulator-semiconductor structure. (b) The band structure at pn-junction and the band-to-band tunneling processes [35].

The B2B tunneling points out the direct tunneling from valance band to the conduction band or the other way round as it is shown in Fig. 3.6(b) at a pn-junction. This process describes the main current transport mechanism in TFETs. B2B tunneling can be controlled with factors such as bias voltage, doping concentration, device structure and material. Thus, it is responsible for the switching of the device and constitutes the current flow in it. Furthermore, considering that these devices are highly doped and doping semiconductors leads to material defects and thus to a high density of traps, in addition to the direct tunneling also TAT plays an important role. The TAT cannot be controlled and is mostly contributed to the off current of the device. In chapter 4 the working principle of TFETs is explained in detail.

\subsection{Tunneling Probability}

As it is mentioned above, the tunneling probability can be determined by solving the $1 \mathrm{D}$ time-independent Schrödinger equation. This differential equation is defined as [49]:

$$
-\frac{\hbar^{2}}{2 m^{*}} \cdot \frac{\mathrm{d}^{2} \Psi(x)}{\mathrm{d} x^{2}}+U(x) \cdot \Psi(x)=E \cdot \Psi(x),
$$

where $\hbar$ presents the reduced Planck's constant, $m^{*}$ is the effective charge carrier mass, $U(x)$ shows the energy barrier, $E$ is the carrier energy and $\Psi(x)$ is the inspected wavefunction of the carrier. Depending on the shape of the barrier, $\Psi$ can be precisely calculated or estimated. Hence, in the following sections the solution of the Schrödinger equation for a rectangular and a triangular energy barrier are considered separately. 


\subsubsection{Rectangular Barrier}

Considering a constant energy barrier as following:

$$
U(x)=\left\{\begin{aligned}
0, & x<x_{1} \\
U_{0}, & x_{1} \leq x \leq x_{2} \\
0, & x>x_{2}
\end{aligned}\right.
$$

which characterizes a rectangular barrier similar to Fig. 3.5, it is possible to solve the Schrödinger equation analytically. The wavefunctions in these three regions are investigated independently. That is to say, the Schrödinger equation is solved for each region separately.

As it is shown in Fig. 3.5, the wavefunction in region $\mathrm{I}\left(\Psi_{\mathrm{I}}\right)$ is actually the superposition of incoming and reflected waves:

$$
\Psi_{\mathrm{I}}(x)=\Psi_{\mathrm{in}}(x)+\Psi_{\mathrm{r}}(x)=A \cdot \exp \left(+\mathrm{j} k_{1} \cdot x\right)+B \cdot \exp \left(-\mathrm{j} k_{1} \cdot x\right)
$$

In this equation, which is in fact the solution of the Schrödinger equation for a free particle, $A$ and $B$ are complex constants and $k_{1}$ represents the wave vector which is defined as:

$$
k_{1}=\sqrt{\frac{2 m^{*} \cdot E}{\hbar^{2}}} .
$$

Assuming that the energy of the particle in region II is lower than the barrier height $\left(E<U_{0}\right)$, the $\Psi_{\mathbf{I I}}$ determines the wavefunction within the energy barrier:

$$
\Psi_{\mathrm{II}}(x)=C \cdot \exp \left(k_{2} \cdot x\right)+D \cdot \exp \left(-k_{2} \cdot x\right)
$$

Here $C$ and $D$ are the complex constants and $k_{2}$ is given by:

$$
k_{2}=\sqrt{\frac{2 m^{*} \cdot\left(U_{0}-E\right)}{\hbar^{2}}} .
$$

On the right side of the barrier similar to region I, the particle can move freely. The only difference to the region $\mathrm{I}$ is that here there is no more energy barrier existing and therefore no reflection needs to be considered. Hence, the solution to the Schrödinger equation in region III is determined as:

$$
\Psi_{\mathrm{III}}(x)=\Psi_{\mathrm{t}}(x)=F \cdot \exp \left(+\mathrm{j} k_{1} \cdot x\right)
$$

In this expression $F$ shows the complex constant and $k_{1}$, which represents the wave vector outside the barrier, is given by Eq. 3.6.

Having the wavefunction in these three regions and considering the continuity and differentiability of the wavefunction, now the boundary conditions at $x=x_{1}$ and $x=x_{2}$ are 
implemented to calculate the constants. Regarding the continuity of the wavefunction at $x=x_{1}$ the following is obtained:

$$
\begin{aligned}
\Psi_{\mathrm{I}}\left(x_{1}\right) & =\Psi_{\mathrm{II}}\left(x_{1}\right) \\
A \cdot \exp \left(+\mathrm{j} k_{1} \cdot x_{1}\right)+B \cdot \exp \left(-\mathrm{j} k_{1} \cdot x_{1}\right) & =C \cdot \exp \left(k_{2} \cdot x_{1}\right)+D \cdot \exp \left(-k_{2} \cdot x_{1}\right)
\end{aligned}
$$

and the differentiability results in:

$$
\begin{aligned}
\frac{\mathrm{d} \Psi_{\mathrm{I}}\left(x_{1}\right)}{\mathrm{d} x} & =\frac{\mathrm{d} \Psi_{\mathrm{II}}\left(x_{1}\right)}{\mathrm{d} x} \\
\mathrm{j} k_{1} \cdot\left(A \cdot \exp \left(+\mathrm{j} k_{1} \cdot x_{1}\right)-B \cdot \exp \left(-\mathrm{j} k_{1} \cdot x_{1}\right)\right) & =k_{2} \cdot\left(C \cdot \exp \left(+k_{2} \cdot x_{1}\right)-D \cdot \exp \left(-k_{2} \cdot x_{1}\right)\right) .
\end{aligned}
$$

Applying the first boundary condition for $x=x_{2}$ leads to:

$$
\begin{aligned}
\Psi_{\mathrm{II}}\left(x_{2}\right) & =\Psi_{\mathrm{III}}\left(x_{2}\right) \\
C \cdot \exp \left(k_{2} \cdot x_{2}\right)+D \cdot \exp \left(-k_{2} \cdot x_{2}\right) & =F \cdot \exp \left(+\mathrm{j} k_{1} \cdot x_{2}\right)
\end{aligned}
$$

and the second one gives:

$$
\begin{aligned}
\frac{\mathrm{d} \Psi_{\mathrm{II}}\left(x_{2}\right)}{\mathrm{d} x} & =\frac{\mathrm{d} \Psi_{\mathrm{III}}\left(x_{2}\right)}{\mathrm{d} x} \\
k_{2} \cdot\left(C \cdot \exp \left(+k_{2} \cdot x_{2}\right)-D \cdot \exp \left(-k_{2} \cdot x_{2}\right)\right) & =\mathrm{j} k_{1} \cdot F \cdot \exp \left(+\mathrm{j} k_{1} \cdot x_{2}\right) .
\end{aligned}
$$

The tunneling probability is actually the ratio of transmitted to the incoming wavefunction and is defined by the squared amplitude of $\Psi_{\mathrm{t}}(x)$ and $\Psi_{\mathrm{in}}(x)$ as following:

$$
T_{\text {tun }}=\frac{\left|\Psi_{\mathrm{t}}(x)\right|^{2}}{\left|\Psi_{\mathrm{in}}(x)\right|^{2}}=\frac{\left|F \cdot \exp \left(+\mathrm{j} k_{1} \cdot x\right)\right|^{2}}{\left|A \cdot \exp \left(+\mathrm{j} k_{1} \cdot x\right)\right|^{2}}=\frac{|F|^{2}}{|A|^{2}} .
$$

If the energy of a particle is lower than the barrier $\left(E<U_{0}\right)$, then by replacing the Eq. (3.10) (3.13) into the Eq. (3.14) the solution to $T_{\text {tun }}$ reads as $[7,8,49]$ :

$$
\begin{aligned}
T_{\mathrm{tun}} & =\frac{1}{1+\left(\frac{k_{1}^{2}+k_{2}^{2}}{2 k_{1} k_{2}}\right)^{2} \cdot \sinh ^{2}\left(k_{2} \cdot\left(x_{2}-x_{1}\right)\right)} \\
& =\frac{1}{1+\frac{U_{0}^{2}}{4 E \cdot\left(U_{0}-E\right)} \cdot \sinh ^{2}\left(\frac{L_{\mathrm{b}}}{\hbar} \cdot \sqrt{2 m^{*} \cdot\left(U_{0}-E\right)}\right)} .
\end{aligned}
$$

The particles with higher energy $\left(E>U_{0}\right)$ do not tunnel through the barrier indeed. The 
transmission mechanism here is called thermionic emission and $T_{\mathrm{tun}}$ is:

$$
\begin{aligned}
T_{\text {tun }} & =\frac{1}{1+\left(\frac{k_{1}^{2}+k_{2}^{2}}{-2 k_{1} k_{2}}\right)^{2} \cdot \sin ^{2}\left(-k_{2} \cdot\left(x_{2}-x_{1}\right)\right)} \\
& =\frac{1}{1+\frac{U_{0}^{2}}{4 E \cdot\left(U_{0}-E\right)} \cdot \sin ^{2}\left(\frac{L_{\mathrm{b}}}{\hbar} \cdot \sqrt{2 m^{*} \cdot\left(U_{0}-E\right)}\right)} .
\end{aligned}
$$

\subsubsection{Triangular Barrier}

When it comes to energy barrier in sense of the band structure of the device, the shape of the barrier is more complex than a rectangle. In consideration of the fact that the barrier associated with TFET is similar to a triangle, here a triangular shape energy barrier (see Fig. $3.7)$ is presented and it is shown how $T_{\text {tun }}$ is attained for it.

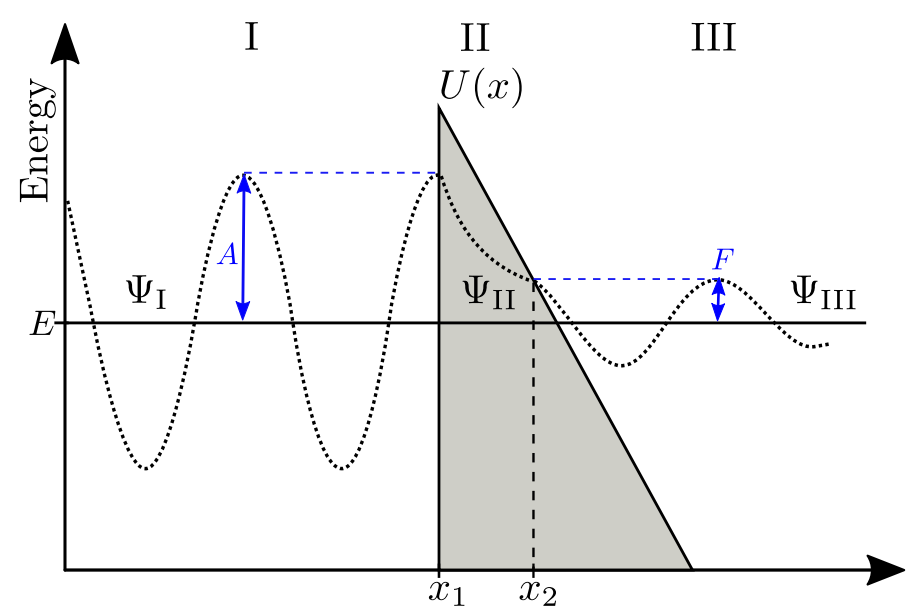

Figure 3.7: The wavefunction of a particle tunneling through a triangular barrier.

For $U$ varying with the position, solving the Schödinger equation and consequently the calculation of the $T_{\text {tun }}$ is complicated and time consuming. Therefore, in this case some methods to estimate the $T_{\text {tun }}$ are developed. Among them Wentzel-Kramers-Brillouin-Approximation known as WKB method is the most used one. This semi-classical approximation is commonly used for quantum mechanics problems where the wavefunction is assumed to be an exponential function similar to the case in region II. Hence, the wavefunction reads as [7]:

$$
\Psi(x)=\Theta(x) \cdot \exp \left( \pm \mathrm{j} k_{3}(x)\right)
$$


with an amplitude of $\Theta(x)$ and a phase of $k_{3}(x)$ which for the case that $E<U(x)$ is defined as:

$$
k_{3}(x)=\sqrt{\frac{2 m^{*} \cdot(U(x)-E)}{\hbar}} .
$$

If $U(x)$ changes very slowly in comparison to the constant de Brogli wavelength $(\lambda=2 \pi / k 3)$, then in a region including many full wavelengths the potential can be considered as constant [53]. In other words, for $\Theta(x)$ and $k_{3}(x)$ both varying slowly, the wavefunction remains practically exponential.

Now in order to see how the WKB method is derived, first the wavefunction is rewritten as $[35]$ :

$$
\Psi(x)=\Theta(x) \cdot \exp (\mathrm{j} \phi(x))
$$

Furthermore, the second derivative of the wavefunction gives:

$$
\frac{\mathrm{d}^{2} \Psi(x)}{\mathrm{d} x^{2}}=\left(k_{3}(x)\right)^{2} \cdot \Psi(x) .
$$

Replacing Eq. (3.19) in Eq. (3.20) leads to:

$$
\frac{\mathrm{d}^{2} \Theta(x)}{\mathrm{d} x^{2}}+2 \mathrm{j} \cdot \frac{\mathrm{d} \Theta(x)}{\mathrm{d} x} \cdot \frac{\mathrm{d} \phi(x)}{\mathrm{d} x}+\mathrm{j} \Theta(x) \cdot \frac{\mathrm{d}^{2} \phi(x)}{\mathrm{d} x^{2}}-\Theta(x) \cdot\left(\frac{\mathrm{d} \phi(x)}{\mathrm{d} x}\right)^{2}=\left(k_{3}(x)\right)^{2} \cdot \Theta(x),
$$

which brings about two separate equations, one for the real part:

$$
\frac{\mathrm{d}^{2} \Theta(x)}{\mathrm{d} x^{2}}-\Theta(x) \cdot\left(\frac{\mathrm{d} \phi(x)}{\mathrm{d} x}\right)^{2}=\left(k_{3}(x)\right)^{2} \cdot \Theta(x) \Rightarrow \frac{\mathrm{d}^{2} \Theta(x)}{\mathrm{d} x^{2}}=\Theta(x) \cdot\left[\left(\frac{\mathrm{d} \phi(x)}{\mathrm{d} x}\right)^{2}+\left(k_{3}(x)\right)^{2}\right]
$$

and the other one for the imaginary part:

$$
2 \mathrm{j} \cdot \frac{\mathrm{d} \Theta(x)}{\mathrm{d} x} \cdot \frac{\mathrm{d} \phi(x)}{\mathrm{d} x}+\mathrm{j} \Theta(x) \cdot \frac{\mathrm{d}^{2} \phi(x)}{\mathrm{d} x^{2}}=0 \Rightarrow \frac{\mathrm{d}}{\mathrm{d} x}\left(\Theta^{2}(x) \cdot \frac{\mathrm{d} \phi(x)}{\mathrm{d} x}\right)=0 .
$$

Regarding Eq. (3.23) it can be concluded that the term $\Theta^{2}(x) \cdot \mathrm{d} \phi(x) / \mathrm{d} x$ is equal to a constant value which links the phase and amplitude of the wavefunction:

$$
\Theta(x)=\frac{C}{\sqrt{\left|\frac{\mathrm{d} \phi(x)}{\mathrm{d} x}\right|}} .
$$


Considering that the amplitude varies slowly with $x$, the second derivative of $\Theta$ is approximately equal to zero. Hence, Eq. (3.22) leads to:

$$
\left(\frac{\mathrm{d} \phi(x)}{\mathrm{d} x}\right)^{2}=-\left(k_{3}(x)\right)^{2} \Rightarrow \phi(x)= \pm \mathrm{j} \int\left|k_{3}(x)\right| \mathrm{d} x
$$

By implementing the solutions for the amplitude and phase the wavefunction is approximated as:

$$
\Psi(x) \cong \frac{C}{\sqrt{\left|k_{3}(x)\right|}} \cdot \exp \left( \pm \int\left|k_{3}(x)\right| \mathrm{d} x\right)
$$

Based on the aforementioned assumptions and results, now the $T_{\text {tun }}$ can be also estimated. Starting with the wave function within the triangular barrier:

$$
\Psi_{\mathrm{II}}(x) \cong \frac{C}{\sqrt{\left|k_{3}(x)\right|}} \cdot \exp \left(+\int_{x}\left|k_{3}(x)\right| \mathrm{d} x\right)+\frac{D}{\sqrt{\left|k_{3}(x)\right|}} \cdot \exp \left(-\int_{x}\left|k_{3}(x)\right| \mathrm{d} x\right),
$$

where $C$ refers to the amplitude of the incoming wave and $D$ represents the amplitude of the reflected part inside the barrier.

Recalling that the tunneling probability is equal to the ratio of the squared amplitude of the transmitted to the incoming wavefunction and considering Fig. 3.7, in this case the $T_{\text {tun }}$ is defined as:

$$
\begin{aligned}
T_{\text {tun }}=\frac{|F|^{2}}{|A|^{2}} & \cong \exp \left(-2 \cdot \int_{x_{1}}^{x 2}\left|k_{3}(x)\right| \mathrm{d} x\right) \\
& =\exp \left(-2 \cdot \int_{x_{1}}^{x 2}\left|\sqrt{\frac{2 m^{*} \cdot(U(x)-E)}{\hbar^{2}}}\right| \mathrm{d} x\right) .
\end{aligned}
$$

It must be kept in mind that this solution is only valid if $U(x)$ varies very slowly. For an energy barrier that varies linearly following equation is valid:

$$
\begin{aligned}
T_{\text {tun }} & =\exp \left(-2 \cdot \int_{x_{1}}^{x_{2}}\left|\sqrt{\frac{2 m^{*}}{\hbar^{2}} \cdot\left(\left[-\frac{U\left(x_{1}\right)-E}{x_{2}-x_{1}} \cdot x+E\right]-E\right)}\right| d x\right) \\
& =\exp \left(-\frac{4}{3} \cdot \sqrt{\frac{2 m^{*}}{\hbar^{2}} \cdot \frac{U\left(x_{1}\right)-E}{x_{1}-x_{2}}} \cdot\left(x_{2}^{3 / 2}-x_{1}^{3 / 2}\right)\right) .
\end{aligned}
$$




\subsection{Poisson's and Laplace's Equations}

The Poisson's equation is a partial differential equation which is widely used for describing the electrical function of field-effect transistors $[8,54]$. It is arisen from the Gaussian law of the Maxwell's equation:

$$
\nabla \cdot \vec{E}(r)=\frac{\rho(r)}{\varepsilon}
$$

in which the divergence of the electric field $\vec{E}(r)$ is related to the charge density $\rho$ and $\varepsilon$ is the constant permittivity of the homogeneous material [35, 54]. For the electric field and potential $\Phi$ in 3D Cartesian coordinates applies:

$$
E(x, y, z)=-\left(\frac{\partial \Phi}{\partial x}+\frac{\partial \Phi}{\partial y}+\frac{\partial \Phi}{\partial z}\right)
$$

Considering the Laplace's operator $(\Delta)$ which is:

$$
\Delta=\frac{\partial^{2}}{\partial x^{2}}+\frac{\partial^{2}}{\partial y^{2}}+\frac{\partial^{2}}{\partial z^{2}}
$$

combining the Eq. (3.30) and Eq. (3.31) results in the Poisson's equation:

$$
\Delta \Phi(x, y, z)=-\frac{\rho(x, y, z)}{\varepsilon} .
$$

The Laplace's equation refers to the special case of the Poisson's equation in which no space charge is existing:

$$
\Delta \Phi(x, y, z)=0
$$

The solutions of the Laplace's equation are known as harmonic functions and in fact there are infinite number of them, yet they must fulfill some conditions. A harmonic function needs to have continuous second-order derivative and hold the superposition principle [54, 55]. Furthermore, the solution to the Laplace's equation must satisfy one of the specific boundary conditions on the enclosing boundary, in a way that maximum and minimum amounts are achieved only on the surface and not within the defined region. In general the boundary conditions can be characterized in three groups [35, 55-57]:

1. Dirichlet condition also known as the boundary value problem of the first kind: the value of the harmonic function $\Phi(x, y, z)$, when it gets close to the edge of the enclosed boundary, on the entire area is predetermined.

2. Neumann condition also known as the boundary value problem of the second kind: the value of the normal derivative of the harmonic function $\vec{n} \cdot \nabla \Phi(x, y, z)$, when it reaches the 
edge of the enclosed boundary, on the entire area is predetermined. Here the $\vec{n}$ describes the normal vector.

3. Mixed condition: a part of the boundary condition is defined by the Dirichlet condition and another part of it with the Neumann condition.

\subsection{Lambert's $W$ function}

In the complex plane, the equation $f(x)=x e^{x}$ cannot be solved elementary to $x$. But by means of some numerical methods such as Newton or bisection method it is possible to approximate the roots of it. The Lambert's $W$ function refers to a series of functions known as branches of this relation and is given as:

$$
x=f^{-} 1\left(x e^{x}\right)=W\left(x e^{x}\right) .
$$

This functions is named after Johann Heinrich Lambert, the swiss scientist, and is also known as omega function or product logarithm. In 1758 he published a series solution of the trinomial equation $x=q+x^{m}$ for $x$. Later in 1783, Euler transformed this equation to a more symmetrical form $\left(x^{a}-x^{b}=(a-b) c x^{(a+b)}\right)$ and he also gave a series solution to this equation which reads [58]:

$$
\begin{aligned}
x^{n}= & +n c+\frac{1}{2} n(n+a+b) c^{2} \\
& +\frac{1}{6} n(n+a+2 b)(n+2 a+b) c^{3} \\
& +\frac{1}{24} n(n+a+3 b)(n+2 a+2 b)(n+3 a+b) c^{4} \\
& +\ldots
\end{aligned}
$$

As the next step Euler considered the case in which $a=b$, and took the limits of his equation $(b \rightarrow a)$. This special case led to the following:

$$
\ln x=c x^{a} .
$$

Then by putting $a=1$ and through the change in variable $z=x^{a}$, he obtained a convergent series solution and finally from this series he derived the standard form of the Lambert's $W$ function (Eq. (3.35)).

Generally it can be said that the Lambert's $W$ function is used to find the solution of transcendental equations. This function has many applications in different fields such as physics, mathematics, quantum statistics and biochemistry. Hence, it is implemented in many programming languages including MATLAB.

There are countably infinite number of branches of the $W$ function. Figure 3.8 illustrates the two real branches of Lambert's $W$ function plotted by MATLAB [59]. It can be seen that for $-e^{-1}<x<0$, the equation $f(x)=x e^{x}$ has exactly two real solutions denoted by $W_{0}(x)$ 
and $W_{-1}(x)$. For $x \geq 0$, the equation $f(x)=x e^{x}$ has exactly one real solution which is $W_{0}(x)$. For $x=-e^{-1}$ this equation has exactly one real solution $W_{0}\left(-e^{-1}\right)=W_{-1}\left(-e^{-1}\right)=-1$. The $W_{0}(x)$ is called the principle branch and its series expansion is defined as following [60]:

$$
\begin{aligned}
W_{0}(x) & =\sum_{n=1}^{\infty} \frac{(-1)^{n-1}(n)^{n-2}}{(n-1) !} x^{n} \\
& =x-x^{2}+\frac{3 x^{3}}{2}-\frac{8 x^{4}}{3}+\frac{125 x^{5}}{24}-\frac{54 x^{6}}{5}+\ldots
\end{aligned}
$$

in which $(n-1)$ ! shows the factorial. It must be mentioned that in this thesis the principal branch of the Lambert's $W$ function in MATLAB is used to do the necessary calculations.

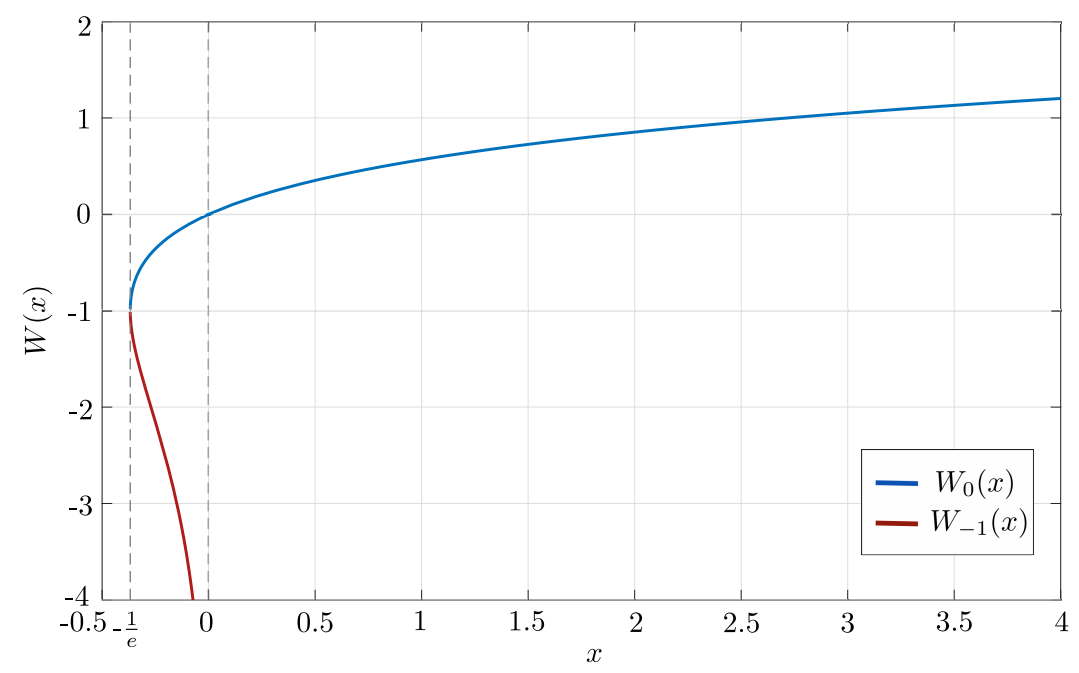

Figure 3.8: The real branches of the Lambert's $W$ function. 


\section{CHAPTER 4}

\section{TFET Essentials}

This chapter focuses on the structure of TFETs. It starts with Sec. 4.1 in which the geometry of the device that is considered in this work and the modeling is done for. In Sec. 4.2 considering the tunneling path in the device, two types of structures are introduced and discussed. Afterwards, with the help of the fundamentals explained in chapter 3 the working principle of TFETs is described in Sec. 4.3. This is followed by the clarification of the transfer characteristics in Sec. 4.4 and the output characteristics of TFETs in Sec. 4.5. The final section of this chapter is dedicated to characterizing the TFET capacitances.

\subsection{Device Geometry}

TFETs are devices with three terminals called source, gate and drain. The drain current in TFETs arises from B2B tunneling. The tunneling probability depends on the band bending and tunneling distance which is controlled by the gate-to-source $\left(V_{\mathrm{gs}}\right)$ as well as the drain-to source $\left(V_{\mathrm{ds}}\right)$ bias [14].

These transistors are designed and also manufactured in various and very individual shapes such as single-gate (SG) planar, NWs and line-tunneling TFETs. In addition to the architecture of a TFET, the material that the device is made of plays an important role in enhancing its functionality. The goal is to increase the control of the gate over the channel region, reduce the unwanted and parasitic effect, such as leakage current and TAT, and all in all improving the performance of TFET.

The structure which is used for the compact modeling in this work is the DG TFET which is depicted in Fig. 4.1. The symmetry of the DG TFET enables the simplicity of the model and allows for implementation of it for other structures.

Depending on the doping type of the source and drain, TFETs are divided in two categories: $\mathrm{n}$-type which refers to the $\mathrm{p}-\mathrm{i}-\mathrm{n}$ structure (see Fig. 4.1(a)) and $\mathrm{p}$-type TFET, the $\mathrm{n}-\mathrm{i}-\mathrm{p}$ structure, is illustrated in Fig. 4.1(b). 


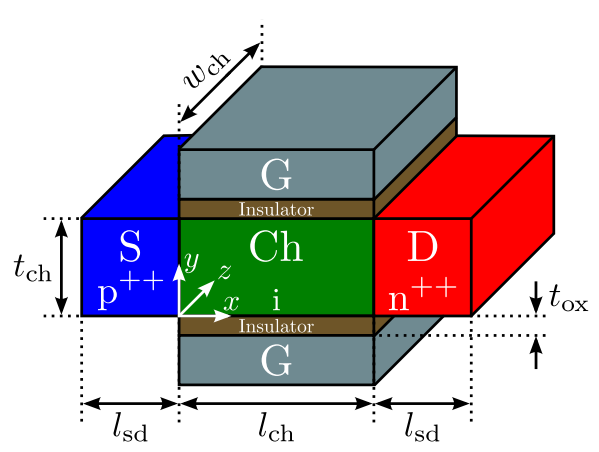

(a) n-type

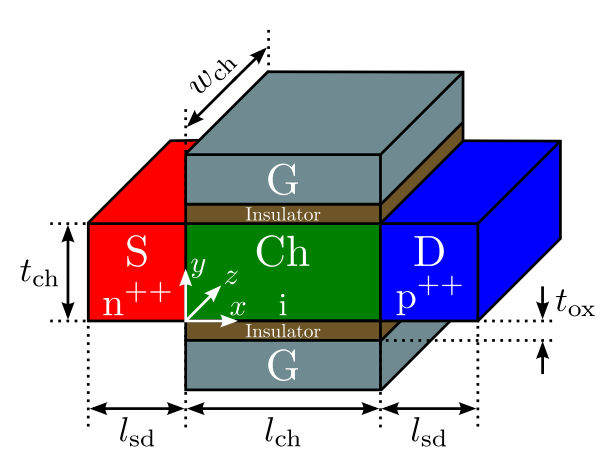

(b) p-type

Figure 4.1: 3D sketch of (a) an n-type and (b) a p-type DG TFET geometry. The structural parameters are defined as follows: $l_{\mathrm{ch}}-$ channel length, $t_{\mathrm{ch}}-$ channel thickness, $w_{\mathrm{ch}}-$ channel width, $t_{\mathrm{ox}}$ - gate insulator (oxide) thickness, $l_{\mathrm{sd}}-$ length of source (S)/drain (D) region. Source and drain are highly doped and the channel is intrinsic.

Here it is considered that the source, drain and channel regions are made of $\mathrm{Si}$, unless another applied material is mentioned. For the gate insulator a high- $\kappa$ material $\left(\mathrm{HfO}_{2}\right)$ is used.

\subsection{Point and Line Tunneling TFET}

Considering the structure of a TFET, the alignment of the electric field which is caused by the gate bias, affects the tunneling direction. In this regard two types of tunneling paths in TFETs are specified: point tunneling and line tunneling [61].

Figure 4.2(a) illustrates a structure in which tunneling mainly occurs in the corner under the gate oxide at the source-channel junction. In fact, the tunneling paths start from source tunnel junction, bend around the aforementioned corner point and come to an end at the oxide-channel interface [61]. That is the reason why this type of tunneling is known point tunneling.

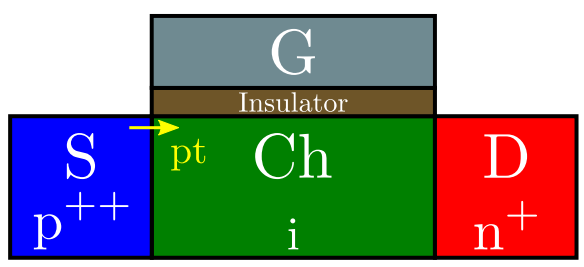

(a)

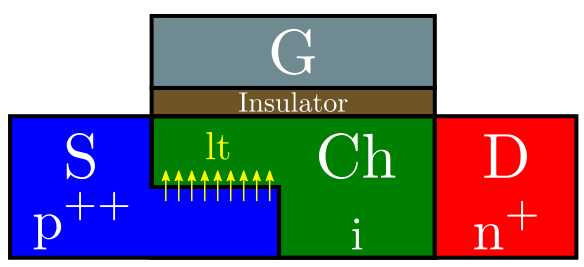

(b)

Figure 4.2: Schematic of (a) n-type point tunneling TFET and (b) n-type line tunneling TFET. Point tunneling occurs at the source-channel interface under the gate oxide. Line tunneling takes place along the channel elongated with the gate's electric field [62]. 
Line tunneling refers to enlargement of the tunneling region. In the structure depicted in Fig. 4.2(b), tunneling happens not only around a little point at the corner, but also along the channel. Considering the gate overlap over the source, the tunneling paths are formed as straight lines in the direction of gate electric field, starting from source junction towards the oxide-channel interface.

In an ideal case, where the parasitic tunneling at edges and the effect of the drain voltage on the source can be neglected, line-tunneling TFETs show a very abrupt turn-on and also a higher on-current $\left(I_{\mathrm{on}}\right)$ in comparison with the point-tunneling TFETs [61, 63]. Hence, this structures are attracting attention to be able to overcome the low $I_{\text {on }}$ problem of point-tunneling devices.

\subsection{Working Principle}

To explain the working principle of TFET, an n-type device is considered in this section. Regarding the geometry of a DG n-type TFET, the band diagram at the position beneath the gate oxide is depicted in Fig. 4.3. By taking into account the effect of applied voltage at TFET terminals on its band structure, three operation states are defined: off-, on- and ambipolar-state.

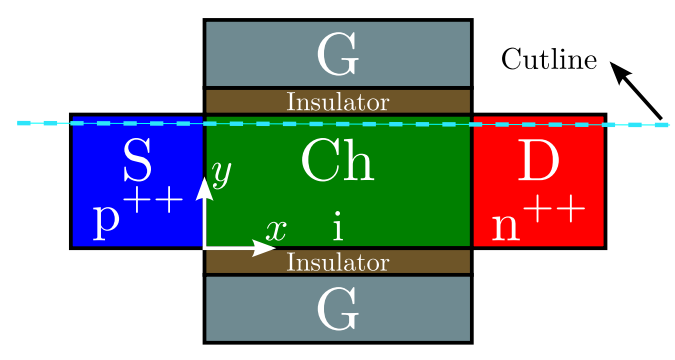

(a)

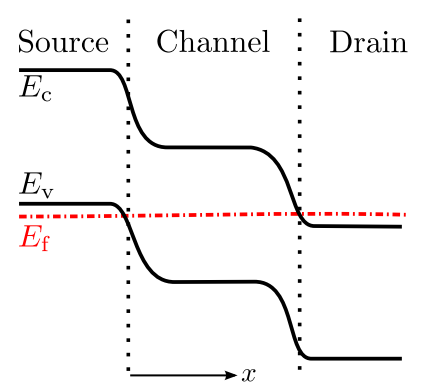

(b)

Figure 4.3: (a) 2D geometry of an n-type TFET. A cutline is made just under the gate oxide along the $\mathrm{x}$-axis. (b) Band diagram of the unbiased device at the $\mathrm{x}$-cutline.

\subsubsection{Off-State}

In this case the drain is connected to $V_{\mathrm{ds}}$ but no gate bias is applied $\left(V_{\mathrm{ds}}>0, V_{\mathrm{s}}=0\right.$ and $V_{\mathrm{gs}}=0$ ). As it can be seen in Fig. 4.4(a) there is no overlap between the valance band of the source and conduction band of the channel or the conduction band of the drain and the valence band of the channel. Therefore, it is expected that the charge carriers can not tunnel into the channel. Anyhow, because of the existence of the traps there is still the probability for carriers to tunnel through the source-channel or drain-channel barrier. The current caused by the TAT in off-state is known as the leakage current or off-current (see Fig. 4.5) [8]. 


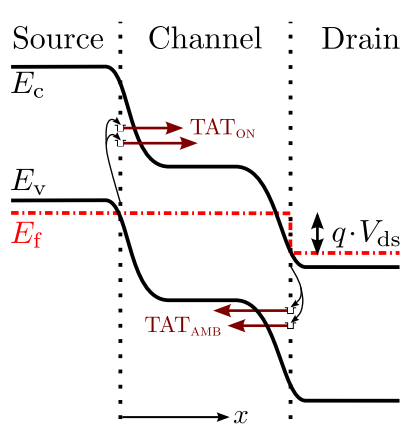

(a) Off-state

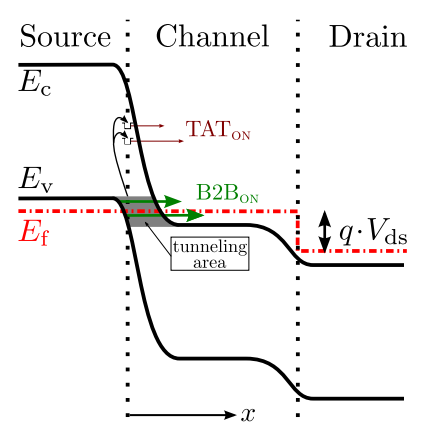

(b) On-state

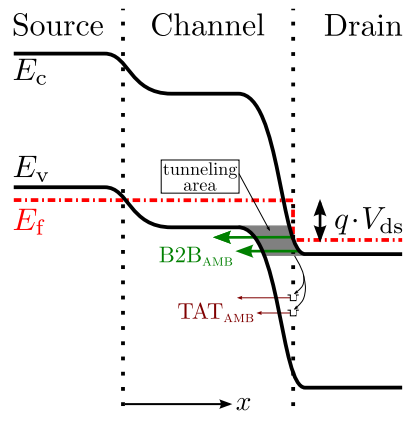

(c) Ambipolar-state

Figure 4.4: Band diagram of an n-type TFET for its different operation states. (a) In offstate there is only TAT occurring at both channel junctions, (b) in on-state additionally to the TAT there is also B2B tunneling at the source-to-channel junction and (c) in ambipolar-state tunneling occurs at the drain-to-channel junction [35].

\subsubsection{On-State}

To turn the TFET on, the charge carries need to flow through the channel. In order to do so, first it is necessary that the electrons tunnel through the potential barrier from source into the channel. Since the source is p-type, the occupied energy states are close to its valance band. Which means, the charge carriers are supposed to tunnel from valance band of the source to the conduction band of the channel [7]. By increasing the gate bias $\left(V_{\mathrm{ds}}>0\right.$ and $\left.V_{\mathrm{gs}}>0\right)$ the band structure of the channel region decreases. At a certain point this increment in the voltage leads to an overlap between the valance band in the source region and the conduction band in the channel (see Fig. 4.4(b)). Hence, carriers can tunnel from source region into the channel, a conduction path is built under the gate oxide and the on-current flows through it. It must be considered that immediately after two bands overlap, the current starts to flow. Therefore, switching from off- to on-state takes place with an abrupt subthreshold swing [8].

By further increase in $V_{\mathrm{gs}}$, the tunneling length becomes shorter and therefore the current increases. Moreover, the inversion charge layer, which is built under the gate oxide due to the gate bias, grows as well. When the magnitude of inversion layer reaches the point that gets comparable with the electron density of the drain, it causes pinning of the channel potential. The channel pinning refers to the incident in which the channel due to the inversion layer is connected to the drain. As the result the channel is shortened and its potential is almost equal to the drain potential. That is to say, after the channel potential is pinned to the drain potential, further increment in $V_{\mathrm{gs}}$ has less influence on the channel potential and consequently in increasing the current [7]. This effect can be seen in Fig. 4.5 in the on-state. 


\subsubsection{Ambipolar-State}

When the gate is negatively biased $\left(V_{\mathrm{ds}}>0\right.$ and $\left.V_{\mathrm{gs}}<0\right)$, the bands of the channel shift upwards. As this negative bias grows, the conduction band of the drain overlaps the valance band of the channel. Figure 4.4(c) depicts the band structure of an n-type TFET in ambipolar-state. In this case holes from conduction band of the drain can tunnel into the channel. This current, which is actually not desired in TFETs, is called ambipolar-current (see Fig: 4.5).

In addition to the holes B2B tunneling in this state, TAT takes place at the drain-to-channel junction as well. Nonetheless, the current caused by the TAT has a very small contribution to the total tunneling current.

As it is already mentioned, ambipolarity is an unwanted effect and increases the switching leakage in TFET inverters. Various approaches have been deployed to suppress the ambipolarity such as reducing the doping concentration of the drain, employing heterostructure with large band gap at drain side to enlarge the tunneling distance and from drain to channel, using heterogeneous gate dielectric and extending the gate over the drain region [64]. So far it seems to be impossible to get rid of this effect completely. However, ambipolarity can be taken as advantage to develop more compact circuits. For instance in [65] it is shown that by employing ambipolarity in designing logic gates, the number of logic levels, delay and power consumption can be reduced. Moreover in [66] it is considered how replacing unipolar transistors by ambipolar transistors affects and simplifies the analog as well as digital circuit design.

\subsection{Transfer Characteristics}

The transfer characteristics of TFETs is a curve which illustrates the output $I_{\mathrm{ds}}$ as a function of $V_{\mathrm{gs}}$. In Fig. 4.5 the transfer characteristics of an n-type TFET, simulated in TCAD Sentaurus for varying drain biases, are shown. In this example different operating regions of the device can be seen.

The constant off-current is due to the traps which are defined at the tunneling junctions of the device. As it is explained in the previous section, by increasing $V_{\mathrm{gs}}$ the tunneling length decreases and therefore, the TAT current rises until the device is switched on and the B2B tunneling takes over. As it is depicted in Fig. 4.5 transferring from off to the on-state occurs abrupt and fast. In this case, the simulated device has a subthreshold swing of almost 30 $\mathrm{mV} / \mathrm{dec}$ which is beyond the limit of $60 \mathrm{mV} / \mathrm{dec}$ for conventional MOSFETs.

By decreasing $V_{\mathrm{gs}}$ to negative voltages the output current again increases and the TFET switches to ambipolar-state. It is comparable with the on-state of a p-type device. In this setting, holes tunnel from drain junction into the channel and rise the $I_{\mathrm{ds}}[61]$.

Figure 4.5 shows that $V_{\mathrm{ds}}$ has a greater influence on the ambipolar-current than on the on-current. The reason is that in the on-state the source junction is the bottleneck for the current and changing the drain bias does not affect the band bending on the source side. Hence, 


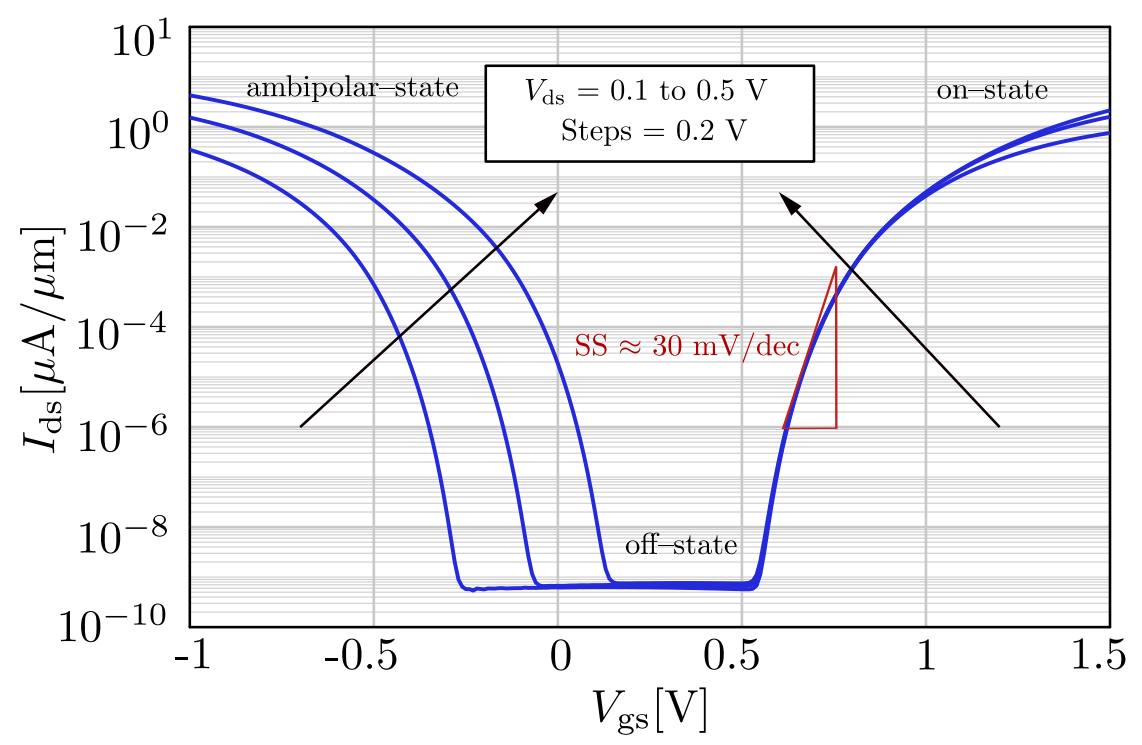

Figure 4.5: Transfer characteristics curves of an n-type TFET are simulated in TCAD for various $V_{\mathrm{ds}}$. The device parameters are defined as following $l_{\mathrm{ch}}=22 \mathrm{~nm}, t_{\mathrm{ch}}=10 \mathrm{~nm}, t_{\mathrm{ox}}=2 \mathrm{~nm}$ and $\mathrm{HfO}_{2}$ is used as insulator material [67].

by varying $V_{\mathrm{ds}}$ no obvious changes can be seen in $I_{\mathrm{ds}}$. On the other hand, in ambipolar-state the drain junction controls the carrier injection in the channel. Considering Fig. 4.4, by increasing $V_{\mathrm{ds}}$ the overlap between the conduction band of the drain and the valance band of the channel becomes bigger. Consequently, the tunneling length become shorter and hence, as it is depicted in Fig. 4.5, the ambipolar-state is reached earlier or in other words, it occurs at higher $V_{\mathrm{gs}}$.

\subsection{Output Characteristics}

The output characteristics refers to a current Vs. voltage curve which depicts the behavior of $I_{\mathrm{ds}}$ as a function of $V_{\mathrm{ds}}$. Figure 4.6 shows the output characteristic curves of an n-type TFET simulated in TCAD Sentaurus. It can be seen that for a fixed $V_{\mathrm{gs}}$, increasing $V_{\mathrm{ds}}$ leads to higher $I_{\mathrm{ds}}$. In order to clarify the reason of this pattern, the case is considered in which the conduction band of the channel falls below the valence band of the source due to the high $V_{\mathrm{gs}}$.

In this situation the transistor is in on-state and the carriers at source junction are injected into the channel and from this point the current is under the control of drain. Hence, the higher $V_{\mathrm{ds}}$ the higher $I_{\mathrm{ds}}$. TFET in this part of the output characteristics is in linear region. This increment continues until $V_{\mathrm{ds}}$ reaches a specific amount known as saturation voltage $\left(V_{\mathrm{ds}, \mathrm{sat}}\right)$. From this point, since the carrier injection has already reached its maximum and the source can not provide the channel with more charges, increasing $V_{\mathrm{ds}}$ can not enhance the current any further. In this condition the TFET is known to be in its saturation region. 


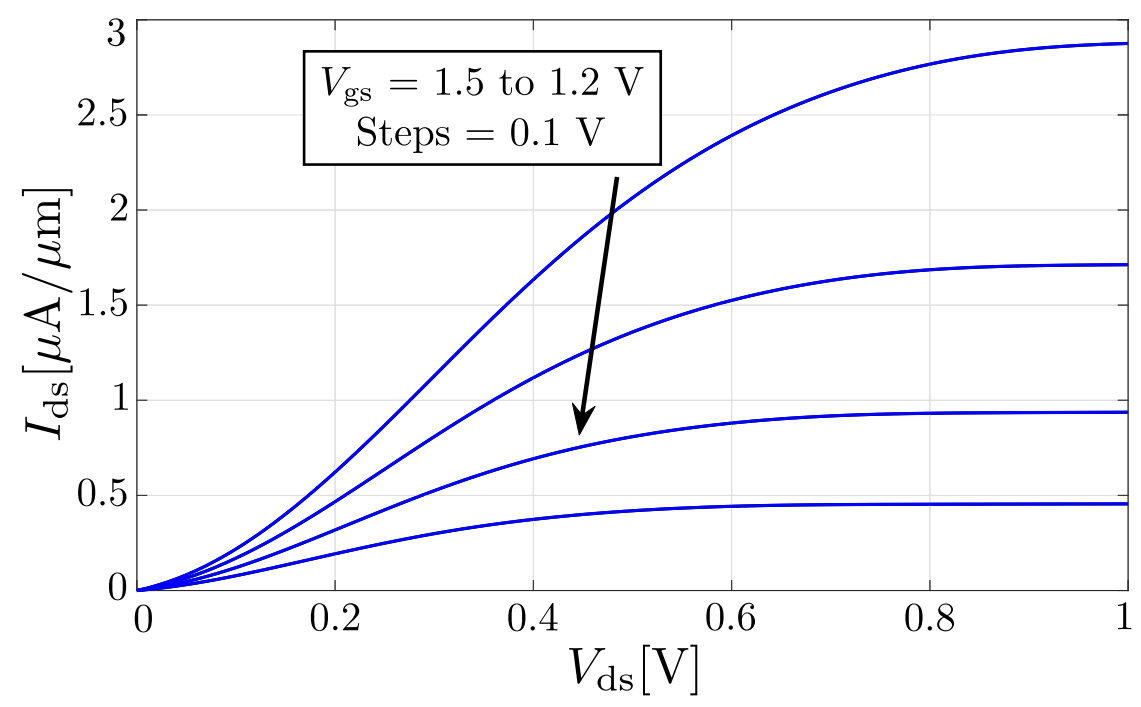

Figure 4.6: Output characteristic curves of an n-type TFET simulated in TCAD for varying $V_{\mathrm{gs}}$. The device parameters are defined as following $l_{\mathrm{ch}}=22 \mathrm{~nm}, t_{\mathrm{ch}}=10 \mathrm{~nm}, t_{\mathrm{ox}}=2 \mathrm{~nm}$ $\mathrm{HfO}_{2}[67]$.

\subsection{Capacitances in TFET}

The transient behavior of a TFET is due to its capacitive components which are caused by the stored charges in the device [1]. Figure 4.8 shows the extrinsic and intrinsic capacitances of a DG TFET which resemble to those in a MOSFET.

\section{Extrinsic Capacitances}

Extrinsic capacitances also known as parasitic capacitances are of two types. Those which are formed as the result of device fabrication and the junction capacitances which are contributed to the overlaps in construction of the transistor:

- In the manufacturing process of TFETs after doping the source and drain region the semiconductor must undergo a high temperature treatment called annealing. This leads to a lateral diffusion of the dopant atoms at the boundary between doped and intrinsic material. Consequently, this border would not be sharp and clear. As the result, the gate overlaps the source and also the drain region. The capacitances $C_{\mathrm{GSO}}$ and $C_{\mathrm{GDO}}$ which are depicted in Fig. 4.8 are caused by this overlap and are called gate overlap capacitances [1].

- The other sort of extrinsic capacitances are those which are formed between bulk and terminals such as $C_{\mathrm{BS}}, C_{\mathrm{BD}}$ and $C_{\mathrm{BG}}$ shown in Fig. 4.8. It must be considered that since TFETs are grown on a wafer with a buried oxide layer, the influence of bulk is negligible. 


\section{Intrinsic Capacitances}

The intrinsic part of the transistor includes channel, oxide, gate, source and drain junction. The capacitances in this portion of device appear by variations of the voltage at TFET terminals and are risen from the channel charges [1].

In DC analysis it is considered that the mobile charges flow from source to the drain. But in the dynamic analysis, in addition to this flow, the charge current generated by stored charges at the terminals must also be taken into account. Figure 4.7 illustrates the dynamic currents at source $\left(i_{\mathrm{s}}\right)$, drain $\left(i_{\mathrm{d}}\right)$ and gate $\left(i_{\mathrm{g}}\right)$ which are associated with the the stored charges at each corresponding terminal [1].

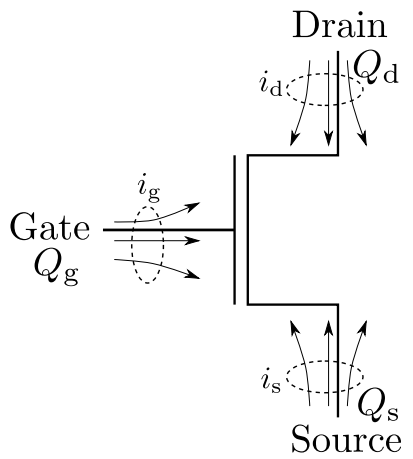

Figure 4.7: Dynamic current associated with the stored charges at the terminals of a TFET $[1]$.

Since the charges at terminals of TFET are a function of applied voltages, the dynamic current flowing through each terminal is formulated as following [1]:

$$
i_{\mathrm{j}}=\frac{d Q_{\mathrm{j}}}{d t}=\frac{\partial Q_{\mathrm{j}}}{\partial V_{\mathrm{g}}} \frac{\partial V_{\mathrm{g}}}{\partial t}+\frac{\partial Q_{\mathrm{j}}}{\partial V_{\mathrm{s}}} \frac{\partial V_{\mathrm{s}}}{\partial t}+\frac{\partial Q_{\mathrm{j}}}{\partial V_{\mathrm{d}}} \frac{\partial V_{\mathrm{d}}}{\partial t}
$$

where $Q$ refers to the charge at the terminal and $\mathrm{j}=\mathrm{g}, \mathrm{s}, \mathrm{d}$. According to this equation, it can be concluded that at every terminal two capacitances corresponding to other terminals known as trans-capacitances and one so called self capacitance are existing. That means in total TFET has nine intrinsic capacitances which show how the charge at the terminal i changes with respect to the applied voltage at terminal $\mathrm{j}$ and by keeping other voltages constant. Generally this capacitances are defined as [1]:

$$
C_{i j}= \begin{cases}-\frac{\partial Q_{i}}{\partial V_{j}}, & i \neq j \quad i, j=g, s, d \\ +\frac{\partial Q_{i}}{\partial V_{j}}, & i=j\end{cases}
$$

All these capacitances can be written together in a matrix as follows: 


$$
C_{i j} \equiv\left[\begin{array}{rrr}
C_{g g} & -C_{g d} & -C_{g s} \\
-C_{d g} & C_{d d} & -C_{d s} \\
-C_{s g} & -C_{s d} & C_{s s}
\end{array}\right]
$$

The positive and negative signs in these expressions refer to the fact that when the voltage at one terminal increases, the charge at that node also increases, and when the voltage at other terminals increases, the amount of charge decreases. Taking into account the charge conservation law in the device, the sum of each row and column of this matrix must be equal to zero [1]. Thus, each self capacitance can be written in terms of two other capacitances as following:

$$
\begin{gathered}
C_{g g}=C_{g s}+C_{g d}=C_{s g}+C_{d g} \\
C_{s s}=C_{s d}+C_{s g}=C_{d s}+C_{g s} \\
C_{d d}=C_{d s}+C_{d g}=C_{s d}+C_{g d} .
\end{gathered}
$$

In Fig. 4.8 the intrinsic part is surrounded by a yellow dashed line. Considering the TCAD simulation results, the gate-drain $\left(C_{\mathrm{gd}}\right)$ and the drain-gate capacitances $\left(C_{\mathrm{dg}}\right)$ as well as the gate-source $\left(C_{\mathrm{gs}}\right)$ and the source-gate capacitances $\left(C_{\mathrm{sg}}\right)$ in TFET are similar. Hence, in this work these capacitances are taken to be equivalent:

$$
\begin{gathered}
C_{s g}=C_{g s}, \\
C_{d g}=C_{g d} .
\end{gathered}
$$

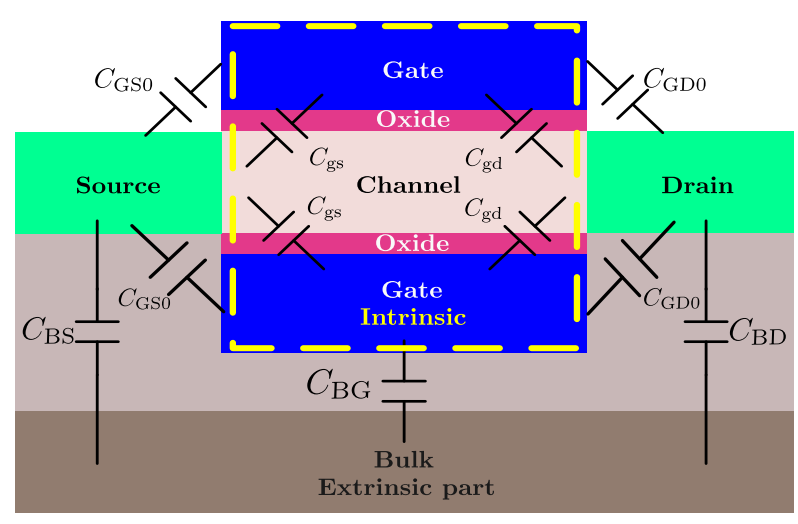

Figure 4.8: Extrinsic and intrinsic capacitances in the structure of a DG TFET. 
It must be mentioned that despite the analogy between TFET and MOSFET structure, there is a significant difference in inversion charge distribution in these two types of transistors. For a MOSFET operating in its linear region both source and drain are connected to the inversion channel. Therefore, the charges in channel are equally assigned to the source and drain which means $C_{\mathrm{gs}}$ and $C_{\mathrm{gd}}$ are equal (see Fig. 4.9). Considering a TFET at $V_{\mathrm{ds}}>0$, for low gate voltage the inversion layer is built up near the drain junction and first by increasing the $V_{\mathrm{gs}}$ this layer is extended towards the source junction. Thus, in a TFET $C_{\mathrm{gs}}$ and $C_{\mathrm{gd}}$ are not equal $[14,36]$.

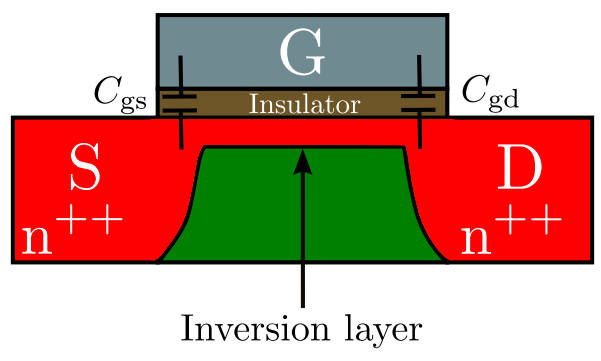

(a) MOSFET

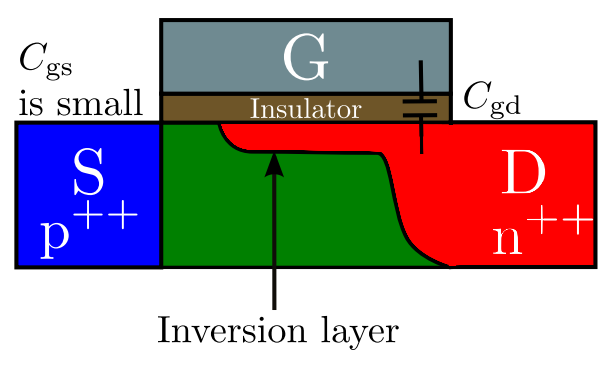

(b) TFET

Figure 4.9: Inversion charge distribution in (a) a MOSFET and (b) in a TFET to the source and channel for $V_{\mathrm{ds}}>0$ and $V_{\mathrm{gs}}>0$ are illustrated [36]. 


\section{CHAPTER 5}

\section{Model Development}

The switching speed of a transistor and generally the dynamic behavior of TFET-based circuits is precisely associated with the capacitances in transistor's construction. Since intrinsic capacitances are much bigger in comparison to the extrinsic ones, they have a greater affect on transient behavior of the TFET. Hence, the focus of this thesis lays on compact modeling of intrinsic capacitances in the TFET structure (see Fig. 4.8).

The compact model is a charge-based model and capacitances are calculated by means of a compact expression for total mobile charge in the channel. In this regard, in Sec. 5.1 the compact expression to calculate the total charge is derived. Considering this expression in Sec. 5.2 intrinsic capacitances are determined. Finally the effect of parasitic elements as well as extrinsic capacitances are added to the model in Sec. 5.3.

\subsection{Charge Calculation}

In [68] the 1D surface potential in TFET is defined as following:

$$
\Phi_{1 \mathrm{D}}=\frac{k_{\mathrm{B}} T}{q} \cdot \ln \left(\frac{N_{\mathrm{ch}} \cdot N_{\mathrm{inv}}}{n_{\mathrm{i}}^{2}}\right),
$$

where $N_{\text {ch }}$ is the channel doping concentration, $N_{\text {inv }}$ shows the inversion charge density and $n_{\mathrm{i}}$ is the intrinsic electron concentration in silicon. The expression $\frac{k_{\mathrm{B}} T}{q}$ is also well-known as the thermal voltage and is shown with $V_{\mathrm{th}}$. Assuming that $\Phi_{1 \mathrm{D}}$ represents an average value for the potential profile from gate to gate, by rearranging the Eq. 5.1, the 1D mobile charge per gate area is expressed as $[57,69]$ :

$$
Q_{\mathrm{m}}^{\prime}=\int_{0}^{t_{\mathrm{ch}}} q \frac{n_{\mathrm{i}}^{2}}{N_{\mathrm{inv}}} \cdot \exp \left(\frac{\Phi_{1 \mathrm{D}}}{V_{\mathrm{th}}}\right) d y=q t_{\mathrm{ch}} \frac{n_{\mathrm{i}}^{2}}{N_{\mathrm{inv}}} \cdot \exp \left(\frac{\Phi_{1 \mathrm{D}}}{V_{\mathrm{th}}}\right) .
$$


Hence, the 1D Poisson's equation in terms of integral inversion charge along the gate to gate line reads as following expression [57, 69, 70]:

$$
C_{\mathrm{eff}}^{\prime} \cdot\left(V_{\mathrm{gs}}-V_{\mathrm{fb}}-\Phi_{1 \mathrm{D}}\right)=\frac{Q_{\mathrm{m}}^{\prime}}{2}=q \frac{t_{\mathrm{ch}}}{2} \frac{n_{\mathrm{i}}^{2}}{N_{\mathrm{inv}}} \cdot \exp \left(\frac{\Phi_{1 \mathrm{D}}}{V_{\mathrm{th}}}\right) .
$$

$V_{\mathrm{fb}}$ presents the flat-band voltage which refers to the voltage that needs to be applied at the gate in order to have flat energy bands at the surface of the semiconductor [1]. $C_{\text {eff }}^{\prime}$ is the effective oxide capacitance per gate area. It is assumed that the charge centroid is located within the channel in a small distance from the $\mathrm{Si}-\mathrm{SiO}_{2}$ interface. Hence, $C_{\text {eff }}^{\prime}$ as it is shown in Fig. 5.1 includes the oxide $\left(C_{\mathrm{ox}}^{\prime}\right)$ and depletion capacitances $\left(C_{\mathrm{dep}}^{\prime}\right)$ per gate area in series [67]:

$$
C_{\mathrm{eff}}^{\prime}=\frac{1}{\frac{1}{C_{\mathrm{ox}}^{\prime}}+\frac{1}{C_{\mathrm{dep}}^{\prime}}}
$$

The derivative of Eq. (5.3) regarding the surface potential leads to:

$$
\frac{\partial V_{\mathrm{gs}}}{\partial \Phi_{1 \mathrm{D}}}=1+\frac{q}{C_{\mathrm{eff}}^{\prime} V_{\mathrm{th}}} \frac{t_{\mathrm{ch}}}{2} \frac{n_{\mathrm{i}}^{2}}{N_{\mathrm{inv}}} \cdot \exp \left(\frac{\Phi_{1 \mathrm{D}}}{V_{\mathrm{th}}}\right)=1+\frac{Q_{\mathrm{m}}^{\prime}}{2 C_{\mathrm{eff}}^{\prime} V_{\mathrm{th}}} .
$$

Now considering Eq. (5.2) and Eq. (5.5), $\frac{\partial Q_{\mathrm{m}}^{\prime}}{\partial V_{\mathrm{gs}}}$ can be written as:

$$
\frac{\partial Q_{\mathrm{m}}^{\prime}}{\partial V_{\mathrm{gs}}}=\frac{\partial Q_{\mathrm{m}}^{\prime}}{\partial \Phi_{1 \mathrm{D}}} \cdot \frac{\partial \Phi_{1 \mathrm{D}}}{\partial V_{\mathrm{gs}}}=\frac{Q_{\mathrm{m}}^{\prime}}{V_{\mathrm{th}}} \cdot \frac{1}{\frac{Q_{\mathrm{m}}^{\prime}}{2 C_{\mathrm{eff}}^{\prime} V_{\mathrm{th}}}+1} .
$$

In order to include the short-channel effect on subthreshold swing in charge calculation an empirical method is introduced in [71]. This approach is derived from the 3D potential solution at the barrier of short-channel silicon-on-insulator (SOI) multi-gate FETs described in [72]. In this method, first the subthreshold swing by means of the surface potential at two

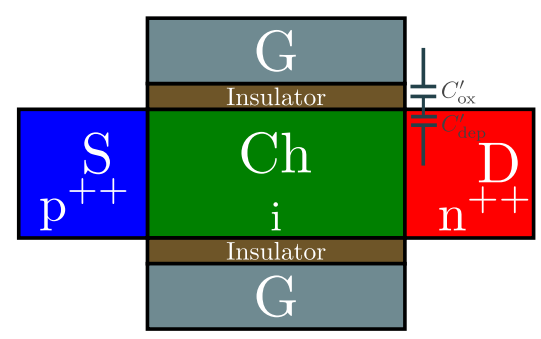

Figure 5.1: The effective oxide capacitance of TFET consists of two capacitances $C_{\mathrm{ox}}^{\prime}$ and $C_{\text {dep }}^{\prime}$ which are placed in series. 
gate potentials $\left(\Phi_{\mathrm{s}}\left(\Phi_{\mathrm{g}}\right)\right)$ with a difference of $d V=0.1 \mathrm{~V}$ is estimated as following:

$$
S=V_{\mathrm{th}} \ln (10) \cdot \frac{d V}{\left.\Phi_{\mathrm{s}}(d V)-\Phi_{\mathrm{s}}(0)\right)} .
$$

Afterward, the slope degradation factor $(\alpha)$, which refers to the ratio between the degraded swing and the ideal subthreshold swing $\left(S_{\text {ideal }}\right)$, is defined as following:

$$
\alpha=\frac{S}{S_{\text {ideal }}}=\frac{S}{60 \mathrm{mV} / \mathrm{dec}} .
$$

It needs to be mentioned that $\alpha$ is used as a fitting parameter in intrinsic capacitance model.

Finally, by including this factor the Eq. (5.6) is modified and results in the following:

$$
\frac{\partial Q_{\mathrm{m}}^{\prime}}{\partial V_{\mathrm{gs}}}=\frac{\partial Q_{\mathrm{m}}^{\prime}}{\partial \Phi_{1 \mathrm{D}}} \cdot \frac{\partial \Phi_{1 \mathrm{D}}}{\partial V_{\mathrm{gs}}}=\frac{Q_{\mathrm{m}}^{\prime}}{V_{\mathrm{th}}} \cdot \frac{1}{\frac{Q_{\mathrm{m}}^{\prime}}{2 C_{\mathrm{eff}}^{\prime} V_{\mathrm{th}}}+\alpha} .
$$

In order to obtain the $\partial V_{\mathrm{gs}}$ in terms of $Q_{\mathrm{m}}$, Eq. (5.9) is rearranged to:

$$
\partial V_{\mathrm{gs}}=\left(\frac{\alpha V_{\mathrm{th}}}{Q_{\mathrm{m}}^{\prime}}+\frac{1}{2 C_{\mathrm{eff}}^{\prime}}\right) .
$$

Aiming to include the effect of the channel width of the device in the charge model, an approximation is made to replace $Q_{\mathrm{m}}^{\prime}$ by $Q_{\mathrm{i}}^{\prime}=Q_{\mathrm{m}, 2 \mathrm{D}}^{\prime} / w_{\mathrm{ch}}$ which is the integral inversion charge normalized regarding the channel width [69]. Thus, the total mobile charge in the channel per gate width can be achieved by integration as follows:

$$
\begin{gathered}
\int_{V_{0}}^{V_{\mathrm{gs}}} d V_{\mathrm{gs}}=\int_{Q_{\mathrm{i}, 0}^{\prime}}^{Q_{\mathrm{i}}^{\prime}}\left(\frac{\alpha V_{\mathrm{th}}}{Q_{\mathrm{i}}^{\prime}}+\frac{1}{2 C_{\mathrm{eff}}^{\prime}}\right) d Q_{\mathrm{i}}^{\prime} \\
V_{\mathrm{gs}}-V_{0}=\alpha V_{\mathrm{th}} \ln \left(\frac{Q_{\mathrm{i}}^{\prime}}{Q_{\mathrm{i}, 0}^{\prime}}\right)+\frac{Q_{\mathrm{i}}^{\prime}-Q_{\mathrm{i}, 0}^{\prime}}{2 C_{\mathrm{eff}}^{\prime}} .
\end{gathered}
$$

To solve this equation for $Q_{\dot{i}}^{\prime}$, firstly by means of the natural logarithm properties it can be rewritten as:

$$
\begin{array}{r}
\ln \left(\exp \left(\frac{2 C_{\mathrm{eff}}^{\prime}\left(V_{\mathrm{gs}}-V_{0}\right)+Q_{\mathrm{i}, 0}^{\prime}}{2 C_{\mathrm{eff}}^{\prime} V_{\mathrm{th}} \alpha}\right)\right)+\ln \left(Q_{\mathrm{i}, 0}^{\prime}\right) \\
=\ln \left(Q_{\mathrm{i}}^{\prime}\right)+\frac{Q_{\mathrm{i}}^{\prime}}{2 C_{\mathrm{eff}}^{\prime} \alpha V_{\mathrm{th}}} .
\end{array}
$$

Now, in order to bring this equation into the form which makes it possible to use the Lambert 
function $\left(x e^{x}=y\right)$, from both sides of the equation $\ln \left(2 C_{\mathrm{eff}}^{\prime} V_{\mathrm{th}} \alpha\right)$ is subtracted and leads to:

$$
\begin{array}{r}
\ln \left(\exp \left(\frac{2 C_{\mathrm{eff}}^{\prime}\left(V_{\mathrm{gs}}-V_{0}\right)+Q_{\mathrm{i}, 0}^{\prime}}{2 C_{\mathrm{eff}}^{\prime} V_{\mathrm{th}} \alpha}\right)\right)+\ln \left(\frac{Q_{\mathrm{i}, 0}^{\prime}}{2 C_{\mathrm{eff}}^{\prime} V_{\mathrm{th}} \alpha}\right) \\
=\ln \left(\frac{Q_{\mathrm{i}}^{\prime}}{2 C_{\mathrm{eff}}^{\prime} V_{\mathrm{th}} \alpha}\right)+\ln \left(\exp \left(\frac{Q_{\mathrm{i}}^{\prime}}{2 C_{\mathrm{eff}}^{\prime} \alpha V_{\mathrm{th}}}\right)\right)
\end{array}
$$

which results in:

$$
\frac{Q_{\mathrm{i}}^{\prime}}{2 C_{\mathrm{eff}}^{\prime} V_{\mathrm{th}} \alpha} \exp \left(\frac{Q_{\mathrm{i}}^{\prime}}{2 C_{\mathrm{eff}}^{\prime} \alpha V_{\mathrm{th}}}\right)=\frac{Q_{\mathrm{i}, 0}^{\prime}}{2 C_{\mathrm{eff}}^{\prime} V_{\mathrm{th}} \alpha} \exp \left(\frac{2 C_{\mathrm{eff}}^{\prime}\left(V_{\mathrm{gs}}-V_{0}\right)+Q_{\mathrm{i}, 0}^{\prime}}{2 C_{\mathrm{eff}}^{\prime} V_{\mathrm{th}} \alpha}\right) .
$$

So, by applying the principle branch of the Lambert's $W$ function [58] to the Eq. (5.15) the expression for the mobile charge per gate area is obtained:

$$
Q_{\mathrm{i}}^{\prime}=2 C_{\mathrm{eff}}^{\prime} V_{\mathrm{th}} \alpha \times W\left(\frac{Q_{\mathrm{i}, 0}^{\prime}}{2 C_{\mathrm{eff}}^{\prime} V_{\mathrm{th}} \alpha} \exp \left(\frac{2 C_{\mathrm{eff}}^{\prime}\left(V_{\mathrm{gs}}-V_{0}\right)+Q_{\mathrm{i}, 0}^{\prime}}{2 C_{\mathrm{eff}}^{\prime} V_{\mathrm{th}} \alpha}\right)\right) .
$$

According to the explanations given in [69], $Q_{\dot{i}, 0}^{\prime}$ is actually referring to the integral inversion charge per gate area for an arbitrary gate bias of $V_{0}$ in the subthreshold regime. Considering the Eq. (5.11), $Q_{\mathrm{i}, 0}^{\prime}$ shows the lower limit of the integral and is supposed to assure the minimum value of the charge in the channel of the transistor. Hence, in the compact model it is calculated as follows:

$$
Q_{\mathrm{i}, 0}^{\prime}=q N_{\mathrm{ch}} t_{\mathrm{ch}}
$$

In the TFET intrinsic capacitance model $V_{0}$ is a fitting parameter that controls when capacitances start to increase. This parameter is defined separately as $V_{0, g s}$ and $V_{0, g d}$ in the calculation of $C_{\mathrm{gs}}$ and $C_{\mathrm{gd}}$, respectively [67].

\subsection{Intrinsic Capacitance Model}

To obtain the capacitances in TFET, the calculations are done separately for the on-state and the ambipolar-state. It is considered that the tunneling barrier in the on-state is located close to the source junction and in the ambipolar-state it is at the drain side. In each case, the mobile charge density at two points of the channel, including the position of the tunneling barrier and the edge of the channel, is computed using the voltage drop between them. By having the charge density at these two points, the total mobile charge of the channel and then the capacitances are calculated. In the following all these calculations are explained in detail.

First, in order to capture the effect of drain and source bias on channel charge, the parameter 
$V$ is implemented in Eq. (5.16) as follows:

$$
Q_{\mathrm{i}}^{\prime}=2 C_{\mathrm{eff}}^{\prime} V_{\mathrm{th}} \alpha \times W\left(\frac{Q_{\mathrm{i}, 0}^{\prime}}{2 C_{\mathrm{eff}}^{\prime} V_{\mathrm{th}} \alpha} \exp \left(\frac{2 C_{\mathrm{eff}}^{\prime}\left(V_{\mathrm{gs}}-V-V_{0}\right)+Q_{\mathrm{i}, 0}^{\prime}}{2 C_{\mathrm{eff}}^{\prime} V_{\mathrm{th}} \alpha}\right)\right) .
$$

Then, to calculate the total channel charge in the on-state, the mobile charge is calculated at the drain junction $\left(Q_{\mathrm{j}, \mathrm{d}}^{\prime}\right)$ and also close to the tunneling barrier on the source side $\left(Q_{\mathrm{b}, \mathrm{s}}^{\prime}\right)$. $Q_{\mathrm{j}, \mathrm{d}}^{\prime}$ and $Q_{\mathrm{b}, \mathrm{s}}^{\prime}$ are obtained by solving the Eq. (5.18) for $V=V_{\mathrm{ds}}$ and $V=V_{\mathrm{ds}}-\Delta V$, respectively. As it is illustrated in Fig. 5.2(a), the tunneling barrier in the on-state is located within the channel in a distance of $X_{\mathrm{b}, \mathrm{s}}$ from source-channel junction. The total mobile charge in the on-state is given as:

$$
Q_{\mathrm{on}}=\frac{Q_{\mathrm{j}, \mathrm{d}}^{\prime}+Q_{\mathrm{b}, \mathrm{s}}^{\prime}}{2} w_{\mathrm{ch}}\left(l_{\mathrm{ch}}-X_{\mathrm{b}, \mathrm{s}}\right) .
$$

In the ambipolar-state, the charge per gate area is once calculated at the source junction $\left(Q_{\mathrm{j}, \mathrm{s}}\right)$ by solving the Eq. (5.18) for $V=V_{\mathrm{s}}$ and then at the tunneling barrier on the drain side $\left(Q_{\mathrm{b}, \mathrm{d}}\right)$ for $V=V_{\mathrm{s}}-\Delta V$. Then the total mobile charge in ambipolar-state is obtained by:

$$
Q_{\mathrm{ambi}}=\frac{Q_{\mathrm{j}, \mathrm{s}}^{\prime}+Q_{\mathrm{b}, \mathrm{d}}^{\prime}}{2} w_{\mathrm{ch}}\left(l_{\mathrm{ch}}-X_{\mathrm{b}, \mathrm{d}}\right)
$$

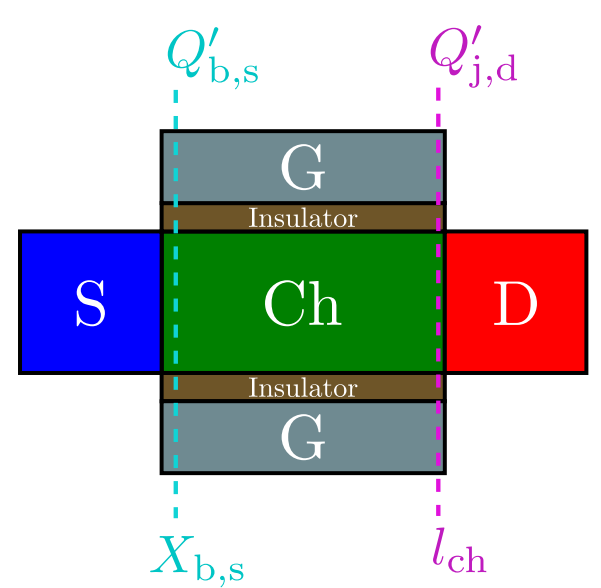

(a)

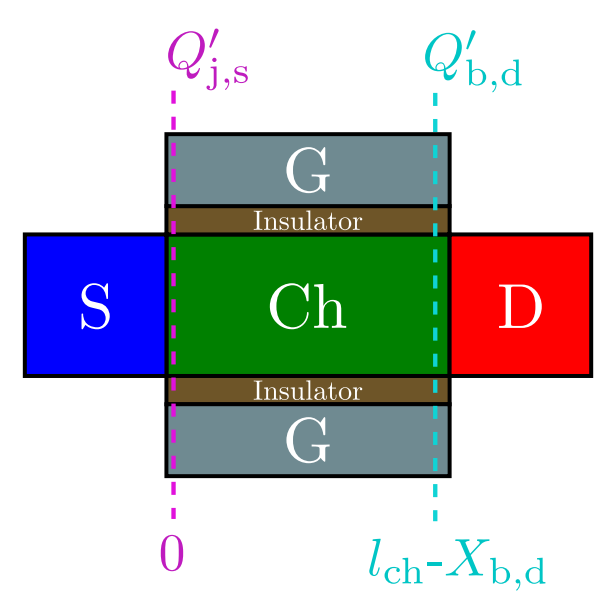

(b)

Figure 5.2: The location of the junction and tunneling barrier in the (a) on-state and the (b) ambipolar-state.

In all these aforementioned calculations $\Delta V$ refers to the voltage drop in the channel. Analysis of long channel TFETs brought us to the conclusion that the effect of voltage drop in the channel needs to be taken into account. Therefore, firstly by means of an empirical method 
the channel charge is calculated by:

$$
Q_{\mathrm{ch}}^{\prime}=Q_{\mathrm{j}, \mathrm{s}}^{\prime}+Q_{\mathrm{j}, \mathrm{d}}^{\prime}+Q_{\mathrm{i}, \mathrm{o}}^{\prime}
$$

in which and $Q_{\mathrm{i}, \mathrm{o}}^{\prime}$ ensures a lower limit for the conductivity in the off-state of the device. Next, the resistivity of the channel between the tunneling barrier and the junction is given as:

$$
R_{\mathrm{ch}}=\frac{l_{\mathrm{ch}}-X_{\mathrm{b}, \mathrm{s}}}{\mu_{\mathrm{n}} Q_{\mathrm{ch}}^{\prime} w_{\mathrm{ch}}}
$$

where $\mu_{\mathrm{n}}$ is the electron mobility. Then, by means of $R_{\mathrm{ch}}$ and $I_{\mathrm{ds}}$ which are calculated using the TFET DC compact model introduced in [35], the voltage drop is achieved as follows:

$$
\Delta V=R_{\mathrm{ch}} I_{\mathrm{ds}}
$$

As it is mentioned in 4.6, in this thesis the focus lays on calculation of the $C_{\mathrm{gs}}, C_{\mathrm{gd}}$ and $C_{\mathrm{gg}}$. So, considering the changes in total mobile charge regarding the change in source or drain voltage, the intrinsic capacitances of TFET in on-state are given as:

$$
\left\{\begin{array}{l}
C_{\mathrm{gs}}=\left.\frac{d Q_{\mathrm{on}}}{d V_{\mathrm{s}}}\right|_{V_{\mathrm{d}}, V_{\mathrm{gs}}} \\
C_{\mathrm{gd}}=\left.\frac{d Q_{\mathrm{on}}}{d V_{\mathrm{d}}}\right|_{V_{\mathrm{s}}, V_{\mathrm{gs}}}
\end{array}\right.
$$

and in ambipolar-state

$$
\left\{\begin{array}{l}
C_{\mathrm{gs}}=\left.\frac{d Q_{\mathrm{ambi}}}{d V_{\mathrm{s}}}\right|_{V_{\mathrm{d}}, V_{\mathrm{gs}}} \\
C_{\mathrm{gd}}=\left.\frac{d Q_{\mathrm{ambi}}}{d V_{\mathrm{d}}}\right|_{V_{\mathrm{s}}, V_{\mathrm{gs}}}
\end{array}\right.
$$

and $C_{\mathrm{gg}}$ results from the summation of $C_{\mathrm{gs}}$ and $C_{\mathrm{gd}}$. Taking into account the symmetrical geometry of the DG TFET and the fact that in this transistors capacitances associated with each gate are in parallel, the capacitances which are calculated hereby indeed characterize the sum of two capacitances. Therefore, in order to adjust the model for the single-gate case, the calculated capacitances from the model need to be halved.

\subsection{Effect of Parasitic Elements on Capacitances}

Comparing the intrinsic capacitance models in Sec. 5.2 to measurement data shows that beside the effect of the voltage drop in the channel there are other parasitic effects existing which need to be considered. Hence, the influence of the parasitic resistances are included in the model. It is assumed that there are series resistances at source and drain terminal of the TFET which 
affect the $C_{\mathrm{gs}}$ and $C_{\mathrm{gd}}$ as follows [73]:

$$
C_{\mathrm{gs}}^{\prime}=C_{\mathrm{gs}}-\left(g_{\mathrm{ds}}+g_{\mathrm{m}}\right) \cdot\left(C_{\mathrm{gs}} R_{\mathrm{s}}-C_{\mathrm{gd}} R_{\mathrm{d}}\right)
$$

and

$$
C_{\mathrm{gd}}^{\prime}=C_{\mathrm{gd}}+g_{\mathrm{ds}} \cdot\left(C_{\mathrm{gs}} R_{\mathrm{s}}-C_{\mathrm{gd}} R_{\mathrm{d}}\right)
$$

Where $C_{\mathrm{gs}}^{\prime}$ and $C_{\mathrm{gd}}^{\prime}$ refer to the capacitances including the effect of parasitic resistances. $R_{\mathrm{s}}$ and $R_{\mathrm{d}}$ are the associated series resistances with the source and drain respectively. $g_{\mathrm{ds}}$ represents the output conductance and $g_{\mathrm{m}}$ is the transconductance which are given as:

$$
g_{\mathrm{ds}}=\frac{\partial I_{\mathrm{ds}}}{\partial V_{\mathrm{ds}}}
$$

and

$$
g_{\mathrm{m}}=\frac{\partial I_{\mathrm{ds}}}{\partial V_{\mathrm{gs}}}
$$

$g_{\mathrm{ds}}$ shows the ratio of the change in drain current to the $V_{\mathrm{ds}}$ changes for constant $V_{\mathrm{gs}}$ and $g_{\mathrm{m}}$ represents the variation in drain current with varying $V_{\mathrm{gs}}$ and a constant $V_{\mathrm{ds}}$ [74]. These parameters are calculated with the help of the TFET compact DC model developed in [35].

Furthermore, analyzing the measured capacitances of a fabricated planar device presented in [75] shed light on the fact that the small signal conductance of the Schottky contacts can also influence the behavior of the intrinsic capacitances.

When in the structure of a TFET, a metal with mid-gap work function with respect to the semiconductor material is used as the contact material, an ohmic contact is expected due to the high doping concentration of the semiconductor at source and drain (see Fig. 5.3). Nevertheless, usually in a TFET structure to suppress the ambipolar current the doping concentration of the drain is reduced. As the result, the small signal conductance of this Schottky contact at the drain side becomes smaller and comparable with the $g_{\mathrm{ds}}$ and $g_{\mathrm{m}}$. This means that the small signal conductance of the Schottky contact, which is in the same order of magnitude as the output and transfer conductance, can also influence the capacitances. In this regard an empirical method is implemented here to show the effect of Schottky contacts on intrinsic TFET capacitances.

In the on-state it is assumed that $C_{\mathrm{gd}}>>C_{\mathrm{gs}}$ and drain has the main impact on the channel charge. Hence, it is expected that only the small signal conductance associated with the drain Schottky contact $\left(g_{\mathrm{d}}\right)$, which is depicted in Fig. 5.3, affect the capacitances as follows:

$$
\begin{gathered}
C_{\mathrm{gs}}^{\prime}=C_{\mathrm{gs}}+C_{\mathrm{gd}} \cdot \frac{g_{\mathrm{m}}}{g_{\mathrm{d}}} \\
C_{\mathrm{gd}}^{\prime}=C_{\mathrm{gd}}-C_{\mathrm{gd}} \cdot \frac{g_{\mathrm{ds}}}{g_{\mathrm{d}}} .
\end{gathered}
$$


In ambipolar-state it is assumed that $C_{\mathrm{gs}}>>C_{\mathrm{gd}}$ and the source controls the channel charge. Therefore, it is considered that the small signal conductance of the source $\left(g_{\mathrm{s}}\right)$ affect the capacitances. So, the intrinsic capacitances in ambipolar-state are defined as:

$$
\begin{aligned}
& C_{\mathrm{gs}}^{\prime}=C_{\mathrm{gs}}-C_{\mathrm{gs}} \frac{g_{\mathrm{ds}}}{g_{\mathrm{s}}} \\
& C_{\mathrm{gd}}^{\prime}=C_{\mathrm{gd}}+C_{\mathrm{gs}} \frac{g_{\mathrm{m}}}{g_{\mathrm{s}}} .
\end{aligned}
$$
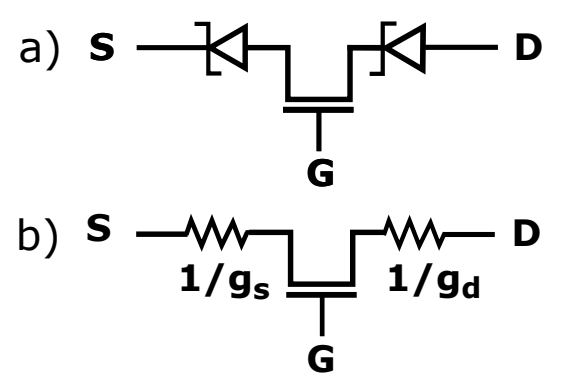

Figure 5.3: Illustration of Schottky barriers at the source and drain of an n-type TFET. (a) To take into account the effect of the Schottky barrier, a Schottky diode is considered at each terminal. (b) The small signal conductance of the diodes are depicted [76].

Both $g_{\mathrm{s}}$ and $g_{\mathrm{d}}$ are fitting parameters. In order to find a realistic range to estimate their values, first some simulations for Schottky diodes are performed in TCAD. Afterwards, by comparing the diode current with the $I_{\mathrm{ds}}$ of the device under investigation, it is considered which Schottky diode with which amount of doping concentration can better characterize the Schottky contact at the barrier of the TFET. Then, the conductance of the chosen diode is calculated and used as the reference to estimate the value of $g_{\mathrm{s}}$ and $g_{\mathrm{d}}$. In the following chapter it is shown with more details how the values for these fitting parameters are picked. 


\section{CHAPTER 6}

\section{Model Verification}

In order to inspect the validity of the compact capacitance model, firstly in Sec. 6.1 the results of the compact model are compared to the simulation outputs which are executed in TCAD Sentaurus [24]. Afterwards, in Sec. 6.2 the model is validated by measurement data of fabricated transistors. In Sec. 6.2.1 the model is tailored to a single-gate (SG) p-type device and its results are compared to the measured data of a fabricated planar TFET. In this part the deviation of the model from the measurements is discussed and a theory to explain and compensate this difference is offered. According to this theory, the parasitic effect of Schottky barrier contacts can strongly affect the TFET capacitances. In order to investigate this theory, it is applied on the TCAD simulation results of an n-type device to check if they show the similar pattern as in measurements. In Sec. 6.2.2 the compact model is employed to characterize the intrinsic capacitances of a Nanowire (NW) and the obtained capacitances are compared to the measured capacitances of this device.

\subsection{Model Verification by Comparison with TCAD Simulations}

For the model verification a 2D DG n-type TFET is simulated in TCAD Sentaurus (see Fig. 4.3(a)). In these simulations a non-local tunneling model for the B2B tunneling current is implemented. To take into account the effect of the TAT current on simulations the Hurks model is used. Moreover, to estimate the band gap narrowing (BGN) in highly p-doped regions the Slotboom model and in highly n-doped regions the Del Alamo model are employed [77, 78]. Considering the BNG in both source and drain affects the on-current and ambipolar-current, respectively.

The B2B tunneling current in the on-state at the source side is calculated with the electron mobility $\mu_{n}=1417 \mathrm{~cm}^{2} / \mathrm{Vs}$, electron tunneling mobility $\mu_{\mathrm{tun}, \mathrm{n}}=3.44 \mathrm{~cm}^{2} / \mathrm{Vs}$, the effective density of states in valance band $N_{\mathrm{v}}=2.65 \cdot 10^{19} \mathrm{~cm}^{-3}$ and the effective electron mass $m_{\mathrm{n}}=0.25 \cdot m_{0}$. To obtain the B2B tunneling current in the ambipolar-state at the drain side, the hole mobility is set to $\mu_{p}=470 \mathrm{~cm}^{2} / \mathrm{Vs}$, tunneling mobility for holes $\mu_{\mathrm{tun}, \mathrm{p}}=3.2 \mathrm{~cm}^{2} / \mathrm{Vs}$, 
the effective density of states in conduction band $N_{\mathrm{c}}=2.8 \cdot 10^{19} \mathrm{~cm}^{-3}$ and the effective hole mass $m_{\mathrm{p}}=0.25 \cdot m_{0}$.

Table 6.1: The parameters used for the TCAD simulations of the short-channel DG n-type TFET.

\begin{tabular}{cc||cc}
\hline Parameter & Value & Parameter & Value \\
\hline$l_{\mathrm{ch}}$ & $22-90 \mathrm{~nm}$ & $w_{\mathrm{ch}}$ & $1 \mu \mathrm{m}$ \\
$t_{\mathrm{ch}}$ & $10 \mathrm{~nm}$ & $N_{\mathrm{s}}, N_{\mathrm{d}}$ & $10^{20} \mathrm{~cm}^{-3}$ \\
$t_{\mathrm{ox}}$ & $2 \mathrm{~nm}$ & Ox. Mat & $\mathrm{HfO}_{2}$ \\
$l_{\mathrm{sd}}$ & $10 \mathrm{~nm}$ & Dev. Mat & Silicon \\
\hline
\end{tabular}

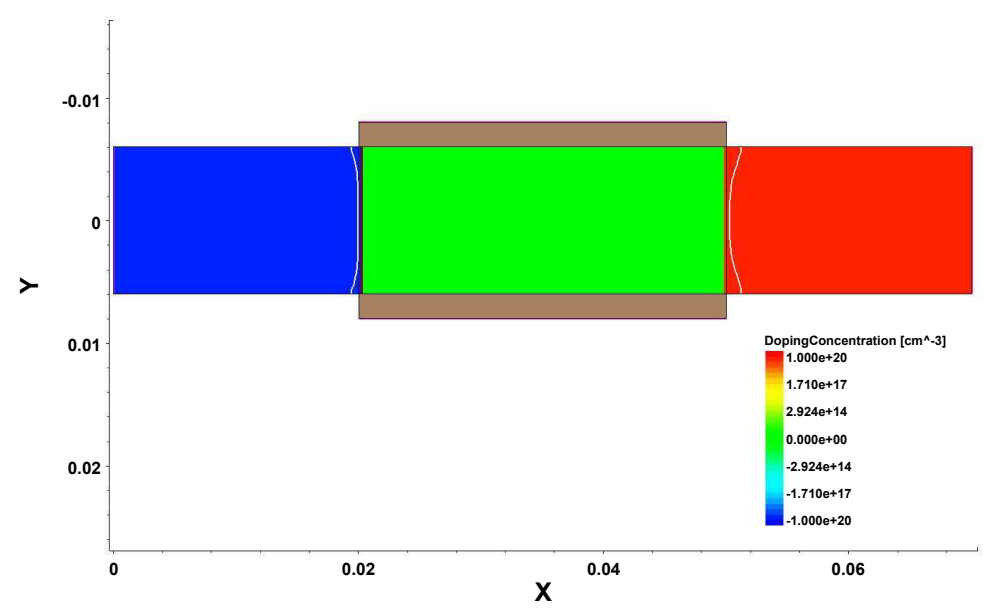

Figure 6.1: The n-type DG TFET structure used for $2 \mathrm{D}$ TCAD simulations. Source is doped with a Boron concentration of $N_{\mathrm{s}}=10^{20} \mathrm{~cm}^{-3}$ and drain with Phosphorus concentration of $N_{\mathrm{d}}=10^{20} \mathrm{~cm}^{-3}$.

To examine the scalability of the compact model, the drain bias and channel length of the device are varied. The 2D structure used for the TCAD simulation is presented in Fig. 6.1 and the variables and parameters are listed in Table 6.1.

Figure 6.2 depicts the $C_{\mathrm{gg}}$ as the function of the gate voltage for varying $V_{\mathrm{ds}}$. The blue solid lines show the results of the model and the dashed black lines are the data resulted from TCAD simulations. Considering the simulated device is an n-type TFET, it is apparent that for $V_{\mathrm{gs}}>0$ and $V_{\mathrm{ds}}>0$ the transistor is in its on-state and for $V_{\mathrm{gs}}<0$ the device is in ambipolar-state. It can be seen that by increasing the drain voltage in on-state, the $C_{\mathrm{gg}}$ decreases or namely shifts. The reason for this is that increasing the $V_{\mathrm{ds}}$ shifts the Fermi potential in the channel and decreases the charge distribution to the drain, thus the device's intrinsic capacitance is reduced.

In Fig. 6.3 the capacitances $C_{\mathrm{gs}}$ and $C_{\mathrm{gd}}$ are illustrated against $V_{\mathrm{gs}}$ at the constant drain bias $\left(V_{\mathrm{ds}}=0.5 \mathrm{~V}\right)$ for varying $l_{\mathrm{ch}}$. When the TFET has a longer $l_{\mathrm{ch}}$, there are more charges 
existing in its channel. For this reason, the longer $l_{\mathrm{ch}}$, the greater the intrinsic capacitances are. In this figure the results obtained from model are compared to those from TCAD simulations. It can be seen that the model is in a good agreement with the TCAD simulations.

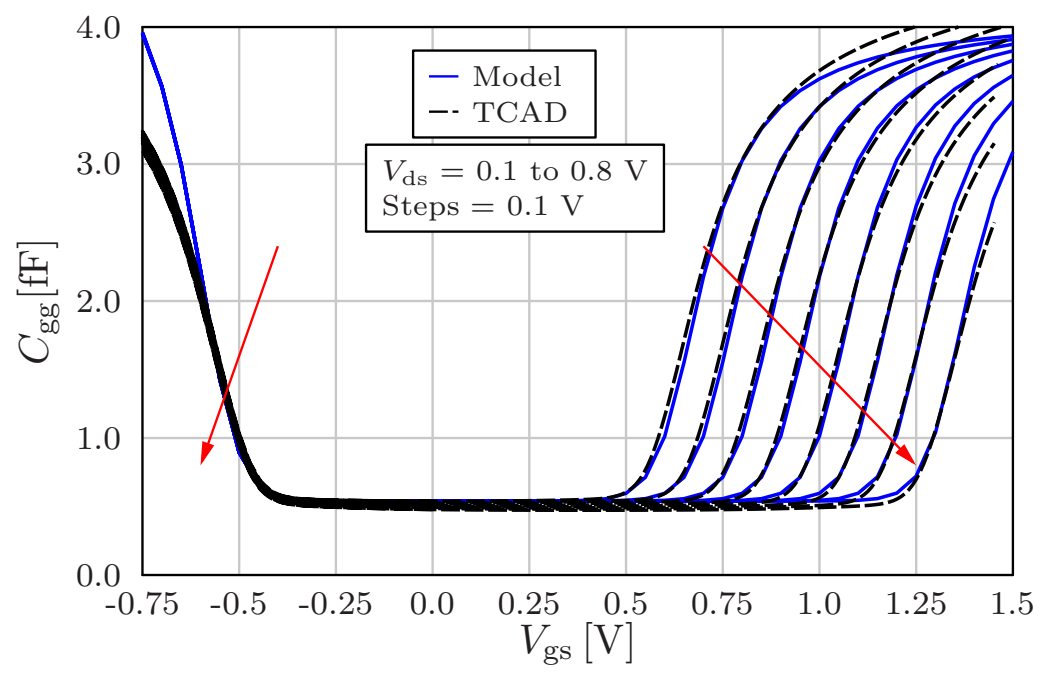

Figure 6.2: $C_{\mathrm{gg}}$ of an n-type DG TFET with $l_{\mathrm{ch}}=22 \mathrm{~nm}$ are compared with the TCAD results for various drain voltages. The fitting parameters of the model are set as $\alpha=1.62$, $X_{\mathrm{b}, \mathrm{s} / \mathrm{d}}=3 \mathrm{~nm}, V_{0, \mathrm{gs}}=-0.19 \mathrm{~V}, V_{0, \mathrm{gd}}=-0.22 \mathrm{~V}$.

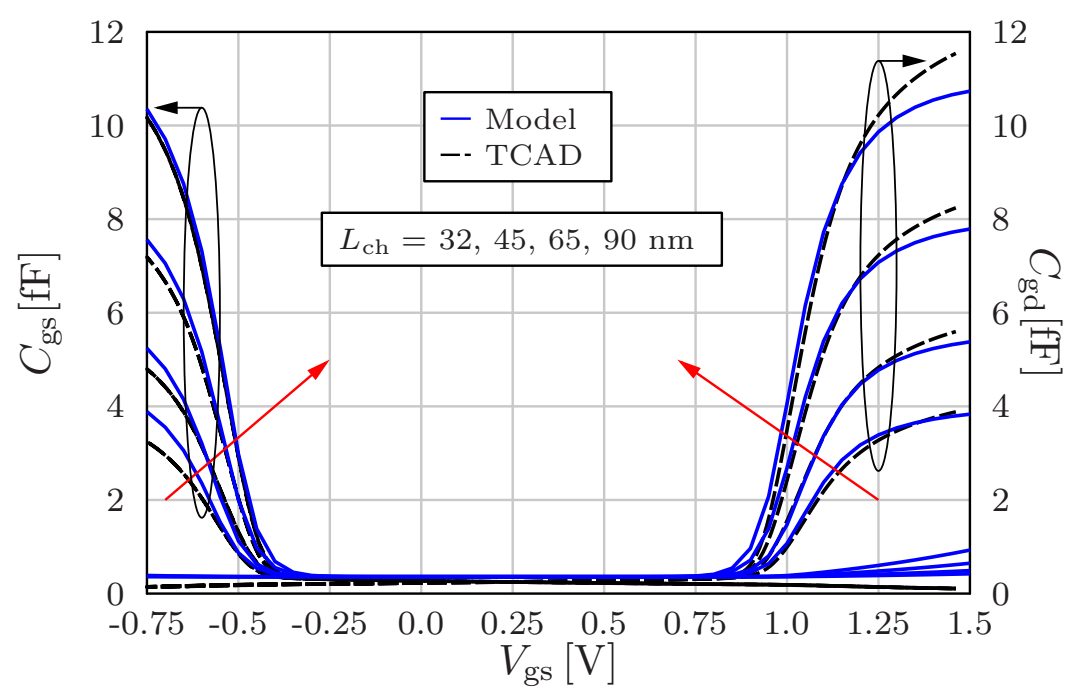

Figure 6.3: $C_{\mathrm{gs}}$ and $C_{\mathrm{gd}}$ of an n-type DG TFET are obtained by the compact model and compared with TCAD simulations for various channel lengths at $V_{\mathrm{ds}}=0.5 \mathrm{~V}$. The fitting parameters are defined as $\alpha=1.53 \ldots 1.61, X_{\mathrm{b}, \mathrm{s} / \mathrm{d}}=3 \mathrm{~nm}, V_{0, \mathrm{gs}}=-0.19 \mathrm{~V}, V_{0, \mathrm{gd}}=-0.22 \mathrm{~V}$. 


\subsection{Model Verification by Comparison with Measurements}

After verifying the intrinsic capacitance model by results of TCAD simulations, to examine the scalability and flexibility of the model, it is applied to calculate the intrinsic capacitances of other structures including a long-channel p-type SG planar TFET and a NW TFET. In each case the results are compared with the measured data, so that the accuracy of the compact model is also investigated.

\subsubsection{Planar TFET}

The geometry of the planar TFET, which is produced in the Forschungszentrum Juelich, is depicted in Fig. 6.4. This device is fabricated on a SOI substrates and the $l_{\mathrm{ch}}=11 \mu \mathrm{m}$, which means it is a long-channel device. Its gate oxide consists of a thin interface layer of $\mathrm{SiO}_{2}$ together with $5 \mathrm{~nm} \mathrm{HfO}_{2}$ and on top of it a layer of $\mathrm{TiN}$ is deposited as the gate contact.

The tunneling junctions which are formed in Si by ion implantation followed by high temperature annealing, lead to an extensive junction and consequently a low tunneling efficiency. Studies have shown that with the help of low energy ion implantation and low temperature annealing process, an abrupt doping profile and therefore a sharp tunneling junction can be formed [79]. Hence, in the structure of the fabricated planar TFET, first, a layer of $\mathrm{NiSi}_{2}$ is deposited on the surface of the Si to form the source and the drain contacts. Afterward, the Phosphor ions are implanted into the source region of this structure with an energy of $3 \mathrm{keV}$, a dose of $2 \times 10^{15} \mathrm{~cm}^{-2}$ and an angle of $45^{\circ}$. In the drain region the Boron ions are implanted with $1.5 \mathrm{keV}$, a dose of $1 \times 10^{15} \mathrm{~cm}^{-2}$ and an angle of $135^{\circ}[75,76]$.

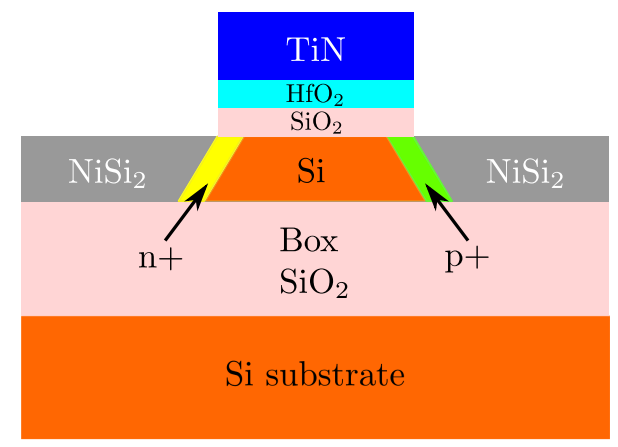

Figure 6.4: Geometry of the fabricated p-type SG planar TFET. The dimensions of the device are as following: $l_{\mathrm{ch}}=11 \mu \mathrm{m}, t_{\mathrm{ch}}=10 \mathrm{~nm}, t_{\mathrm{ox}}=5 \mathrm{~nm}$ and $w_{\mathrm{ch}}=10 \mu \mathrm{m}$ [75].

In Fig. 6.5 the measured capacitances of the planar structure for different $V_{\mathrm{ds}}$ and as a function of $V_{\mathrm{gs}}$ are depicted. Since the TFET is p-type for $V_{\mathrm{gs}}<0$ it is in the on-state and for $V_{\mathrm{gs}}>0$ it in the ambipolar-state. The increase in $V_{\mathrm{ds}}$ shifts the capacitance curves, which meets the expectations. As it is mentioned in Sec. 6.1, this effect occurs due to the charge distribution to the drain. Considering the simulated capacitances of an n-type device, it was 
expected that $C_{\mathrm{gd}}$ would also be the dominant capacitance for the p-type case in the on-state. But the measured capacitances show an unexpected pattern. It can be seen that in the on-state not only the $C_{\mathrm{gd}}$ increases, but also the $C_{\mathrm{gs}}$ starts to grow. This effect can be seen in the ambipolar state as well, but on a lower magnitude. This means that in this case, in addition to $C_{\mathrm{gs}}, C_{\mathrm{gd}}$ is also increased, however this increment is not as big as the one for on-state. Furthermore, in Fig. 6.5 it can be seen that as the negative gate bias grows, $C_{\text {gs }}$ gets higher and after that $V_{\mathrm{gs}}$ reaches a specific amount, it starts to decrease.

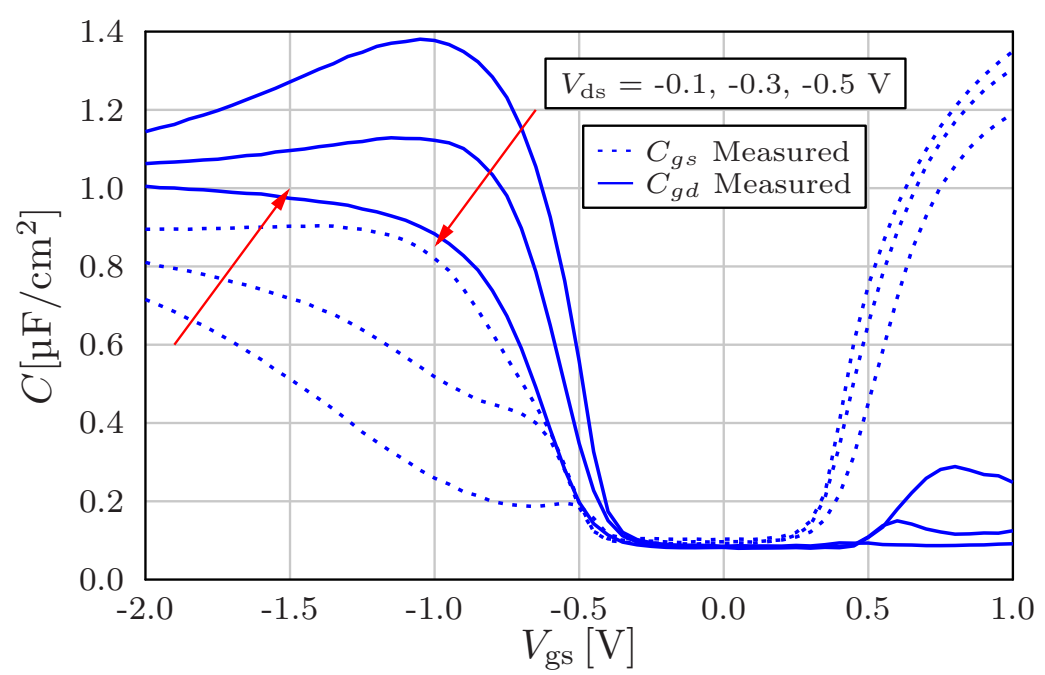

Figure 6.5: The intrinsic capacitances $C_{\mathrm{gs}}$ and $C_{\mathrm{gd}}$ of the fabricated p-type planar TFET are measured by varying $V_{\mathrm{gs}}$ at different $V_{\mathrm{ds}}[76]$.

In order to justify this unexpected pattern appearing in measurements, some investigations are carried out to check whether the inclusion of parasitic effects, which do not add complexity to the model and yet make physical sense, compensates this effect in the model.

The very first guess was that due to the long channel of the device the voltage in the channel drops drastically and leads to a change in the charge distribution to the source and drain. To be more specific, regarding the assumption that in TFET the tunneling barrier is the bottleneck for the current, the charge carriers in the on-state from the point that they are injected into the channel are supposedly attributed to the drain. In the case of the long-channel, it is suspected that this charge propagation cannot be employed. It seems that channel charges in on-state, are not only distributed to the drain, rather to both source and drain.

For the purpose of examining this hypothesis, in the compact model the channel voltage drop was multiplied by a constant. As a result of the multiplication of $\Delta V, C_{\mathrm{gs}}$ was increased in the on-state and with this increase $C_{\mathrm{gd}}$ started to decrease. In addition, in the ambipolar-state, $C_{\mathrm{gd}}$ began to increase while $C_{\mathrm{gs}}$ began to decrease. However, the pattern of the aforementioned changes in the behavior of the capacitances was still different from measurements data. 
Studying the small signal analysis of standard MOSFETs has shown that the parasitic resistances at the source and drain contacts affect the intrinsic capacitances. In [73] to address this effect on the MOSFET capacitance in a compact dynamic model, a simple method is introduced. Hence, the next presumption was that by transferring this method to the TFET model, it would probably get closer to the measured data. The effect of parasitic resistances is implemented into the model as it is explained in Sec. 5.3. This method has helped to improve the model, however there are still some deviations from measurements. So, it has brought us to investigating the effect of Schottky barrier contact on intrinsic capacitances.

In spite of that $\mathrm{NiSi}_{2}$ has a mid-gap work function with reference to silicon, at the interface of these two materials an Ohmic contact is expected. That is to say, considering the fact that the source and drain regions in the TFET are highly doped, it is assumed that the charge carriers simply tunnel through the Schottky barrier, because the tunneling length is very low due to the high band bending. But as it is addressed in Sec 5.3, the reduced drain doping concentration leads to a lower small signal conductance at the drain side and affects the intrinsic capacitances. In order to include the impact of the Schottky barrier contact into the model, an empirical approach is developed. To see how this method is implemented in the compact model, it is asked to refer to the Sec. 5.3.

It should be noted that for the purpose of assessing all these hypotheses, they are also implemented and simulated in TCAD Sentaurus [24], ATLAS [25] and Minimos-NT [26]. However, these simulations can not genuinely reproduce the behavior of the measured capacitances. The TCAD simulations considering two Schottky barriers at source and drain show a deficient convergence. Nevertheless, they show results that indicated a tendency, but are not in complete agreement with the expectations [76].

To overcome the convergence problem and estimating the $g_{\mathrm{d}}$ and $g_{\mathrm{s}}$, which regarding the Eq. (5.30) to Eq. (5.33) are required, firstly some Schottky diodes with different semiconductor doping concentrations are simulated in TCAD. In these simulation Boron is used as the doping material. The current and the small signal conductance of these diodes at a low bias are illustrated in Fig. 6.6. In Fig. 6.7 the compact DC model [35] is fitted to the measured $I_{\mathrm{ds}}$ of the planar TFET. A comparison of these two plots shows that for Schottky diodes with a drain doping concentration lower than $1 \times 10^{19} \mathrm{~cm}^{-3}$, the current is in the same range as $I_{\mathrm{ds}}$ in the fabricated TFET. Considering the case in which the drain doping is equal to $1 \times 10^{19} \mathrm{~cm}^{-3}$, the small signal conductance of the Schottky barrier contact at a voltage of $0 \mathrm{~V}$ is almost $5 \times 10^{-5} \mathrm{~S} / \mu \mathrm{m}$. To put it in another way, these simulations show the range of the small signal resistance of the Schottky barrier and help to make a more realistic estimate of the value of $g_{\mathrm{d}}$ and $g_{\mathrm{s}}$. 


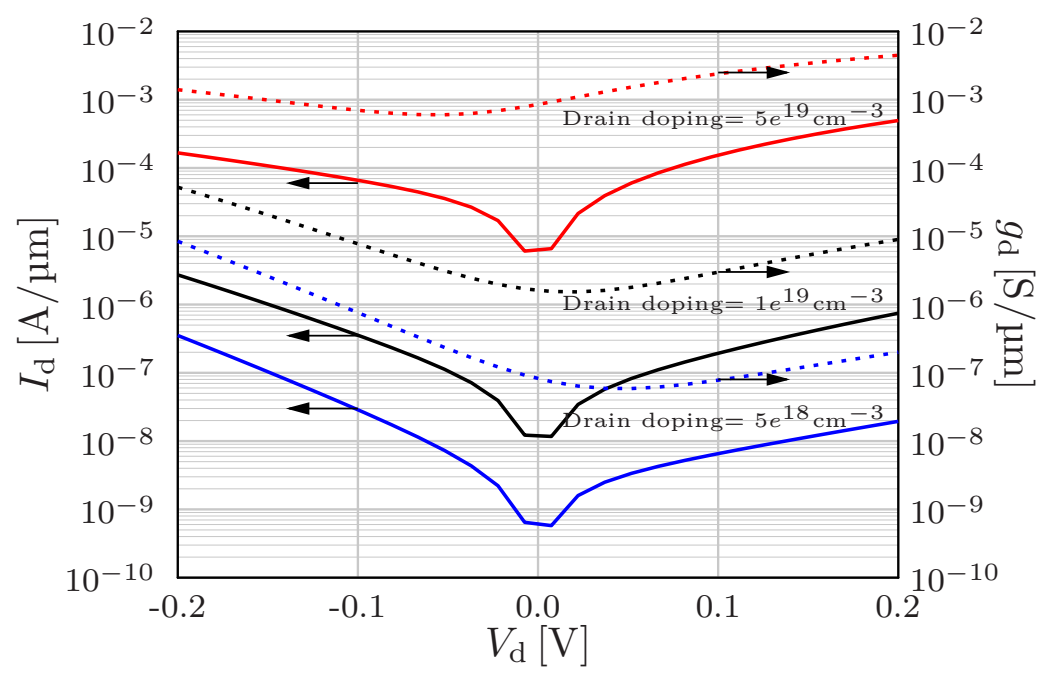

Figure 6.6: Schottky diodes are simulated in TCAD to estimate the small signal conductance of the Schottky barrier contacts at the source and drain of the TFET. In each case the semiconductor part of the diode is doped with different concentrations of Boron. Solid lines depict the diode current and the dotted lines show the small signal conductance of the diodes [76].

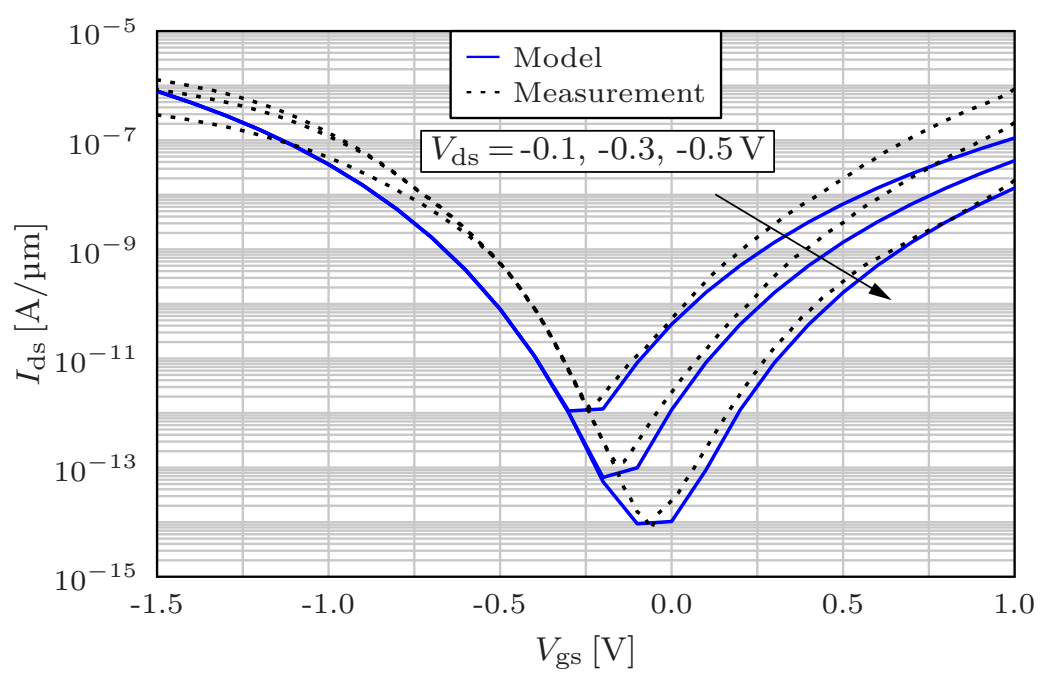

Figure 6.7: The compact DC model [35] is fitted to the measured drain current in the p-type single-gate planar TFET. 
To monitor the effect of this theory on the simulated intrinsic capacitance, it is decided to simulate a TFET in TCAD to extract both DC and AC results and then with the help of the compact model apply the Schottky barrier effect on those results. To do so, firstly the transfer and output characteristics of an n-type short-channel device are simulated in TCAD (see Fig. 6.8). The structure of the simulated TFET is equal to the one shown in Fig. 6.1 with $l_{\mathrm{ch}}=22 \mathrm{~nm}$ and the other parameters are considered to be the same as what is listed in Tab. 6.1. From these simulations $g_{\mathrm{ds}}$ and $g_{\mathrm{m}}$ as well as intrinsic capacitances are obtained. In Fig. 6.8(b) in addition to the $I_{\mathrm{ds}}$ also $g_{\mathrm{ds}}$ as a function of $V_{\mathrm{ds}}$ for various $V_{\mathrm{gs}}$ is depicted. It can be seen that $g_{\mathrm{ds}}$ by increasing $V_{\mathrm{ds}}$, firstly tends to increase but then it sinks. In Fig. 6.9(a), $g_{\mathrm{ds}}$ is shown on the left axis as a function of $V_{\mathrm{gs}}$ for different $V_{\mathrm{ds}}$. For the positive gate bias, or in other words, in the on-state, $g_{\mathrm{ds}}$ shows a higher value at lower drain biases. On the other hand in the ambipolar-state, at higher $V_{\mathrm{ds}}, g_{\mathrm{ds}}$ has a greater value. Additionally, on the right axis the ratio between $g_{\mathrm{ds}}$ and $g_{\mathrm{d}}$ in the on-state and $g_{\mathrm{ds}}$ and $g_{\mathrm{s}}$ in the ambipolar-state are presented. Based on the results of the simulations which are performed for the Schottky diodes, the small signal conductances are chosen as $g_{\mathrm{d}}=8.33 \times 10^{-5} \mathrm{~S} / \mu \mathrm{m}$ and $g_{\mathrm{s}}=1 \times 10^{-4} \mathrm{~S} / \mu \mathrm{m}$. As it is already mentioned, these values are just estimated and used as fitting parameters. 


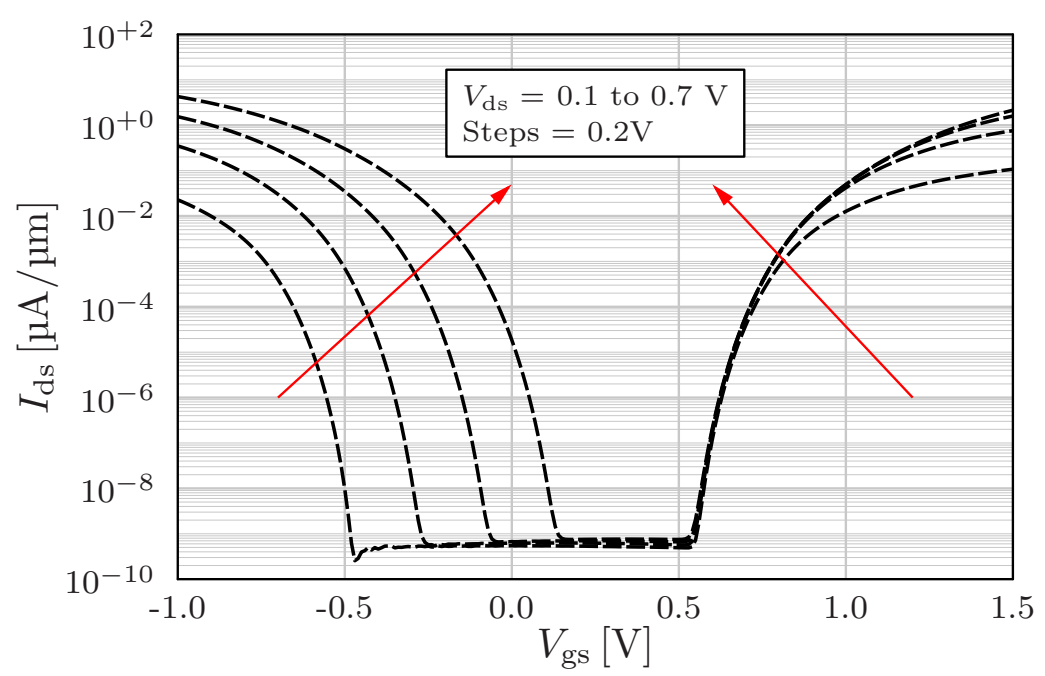

(a)

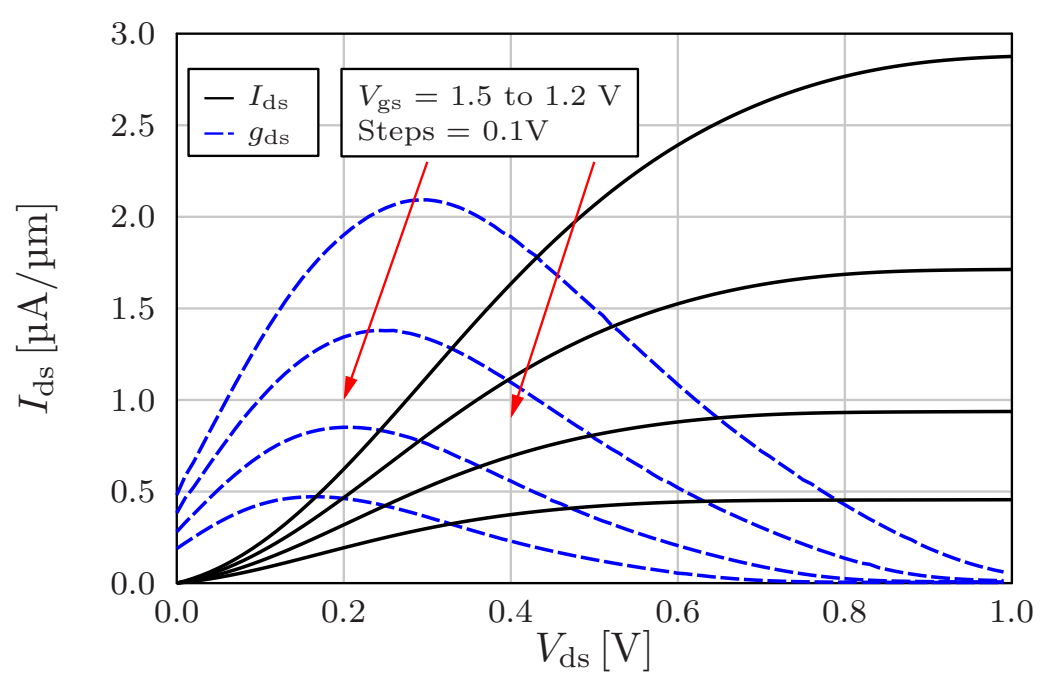

(b)

Figure 6.8: (a) Transfer characteristic of the $n$-type TFET simulated in TCAD for varying $V_{\mathrm{ds}}$. (b) Output characteristic curves of the n-type TFET simulated in TCAD for various $V_{\mathrm{gs}}$ together with their corresponding $g_{\mathrm{ds}}$ curves [76]. The geometry of the simulated device is shown in Fig. 6.1. The dimensions of the TFET are set as following: $l_{\mathrm{ch}}=22 \mathrm{~nm}, t_{\mathrm{ch}}=10 \mathrm{~nm}$, $t_{\mathrm{ox}}=2 \mathrm{~nm} \mathrm{HfO}_{2}$. 


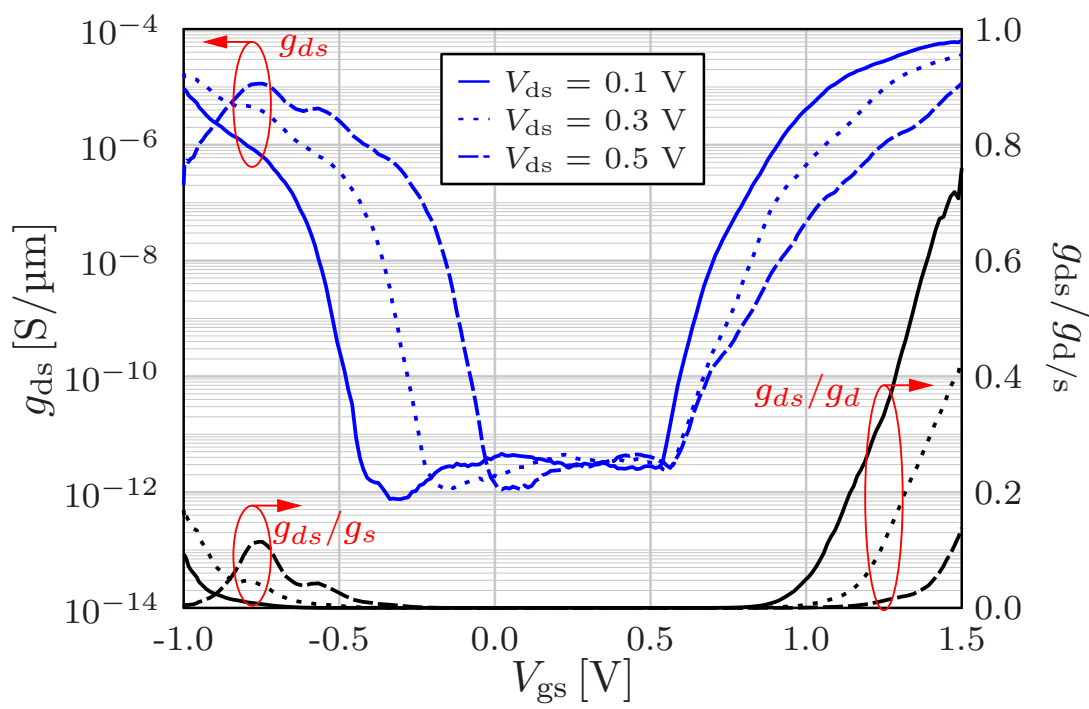

(a)

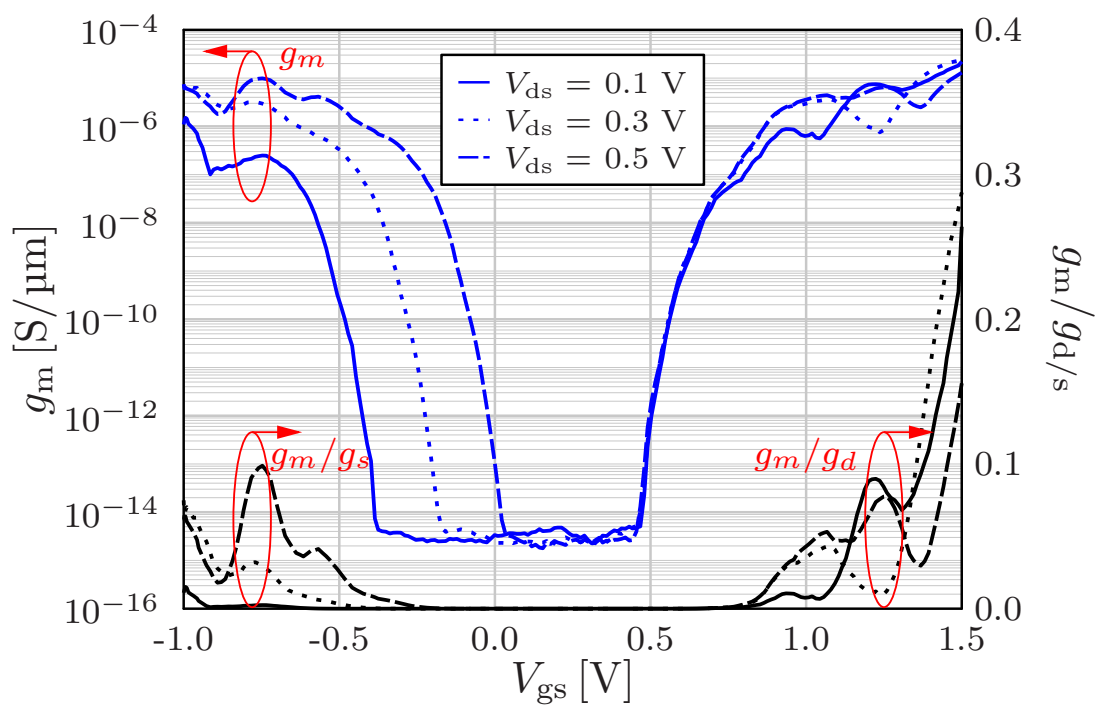

(b)

Figure 6.9: (a) The output conductance of a short-channel DG n-type TFET extracted from TCAD simulations. On the right axis $g_{\mathrm{ds}}$ is shown, which is normalized in the on-state with respect to $g_{\mathrm{d}}$ and in the ambipolar-state with respect to $g_{\mathrm{s}}$. (b) Transconductance of the $\mathrm{n}-$ type TFET is extracted from AC analyzes in TCAD. On the right axis the normalized value of $g_{\mathrm{m}}$ regarding $g_{\mathrm{d}}$ in the on-state and to $g_{\mathrm{d}}$ in the ambipolar-state are shown [76]. 
Considering all these analyses and according the Eq. (5.31) it is expected that the Schottky barrier in on-state for the lower drain biases has a stronger affect on $C_{\mathrm{gd}}$. Moreover, by taking into account Eq. (5.32) it can be concluded that in the ambipolar-state the small signal resistances of Schottky barrier affect the most $C_{\mathrm{gs}}$ when the drain bias is lower.

In Fig. 6.9(b) on the left axis $g_{\mathrm{m}}$ against $V_{\mathrm{gs}}$ for varying $V_{\mathrm{ds}}$ is presented. On the right axis the normalized value $g_{\mathrm{m}} / g_{\mathrm{d}}$ in the on-state and $g_{\mathrm{m}} / g_{\mathrm{s}}$ in the ambipolar-state are shown. In the on-state by varying $V_{\mathrm{ds}}$ not a significant change is observed in $g_{\mathrm{m}}$. But in the ambipolar-state it can be seen that for the higher $V_{\mathrm{ds}}, g_{\mathrm{m}}$ is bigger. Considering the normalized values of $g_{\mathrm{m}}$ regarding $g_{\mathrm{d}}$ and $g_{\mathrm{s}}$ shown on the right axis, it can be said that in the on-state the effect is stronger. However, comparing Fig. 6.9(a) with 6.9(b) it appears that the normalized $g_{\mathrm{ds}}$ has a stronger effect on capacitances than the normalized $g_{\mathrm{m}}$.

After analyzing the behavior of $g_{\mathrm{ds}}$ and $g_{\mathrm{m}}$ in the presence of the small signal conductances of the Schottky barrier contacts, the intrinsic capacitances are finally calculated as explained in section 5.3 of the previous chapter. In Fig. 6.10 the TFET capacitances $C_{\mathrm{gs}}^{\prime}$ and $C_{\mathrm{gd}}^{\prime}$ are presented in comparison to the case where the effect of the Schottky barrier contacts is neglected. The blue solid lines show $C_{\mathrm{gs}}^{\prime}$ and $C_{\mathrm{gd}}^{\prime}$ and the black dotted curves depict $C_{\mathrm{gs}}$ and $C_{\mathrm{gd}}$. By incorporating the effect of the Schottky barrier into the TCAD simulation results in the on-state, at a certain gate bias highlighted with a red circle, $C_{\mathrm{gd}}^{\prime}$ starts to fall and $C_{\mathrm{gs}}^{\prime}$ starts to rise. Considering this bias and comparing it to Fig. 6.9, it can be seen that where the $g_{\mathrm{ds}} / g_{\mathrm{d}}$ and $g_{\mathrm{m}} / g_{\mathrm{d}}$ curves begin to rise is actually where the effect of the Schottky barrier contact on capacitances is apparent. The similar pattern can also be observed in the ambipolar-state where $C_{\mathrm{gd}}^{\prime}$ increases and $C_{\mathrm{gs}}^{\prime}$ decreases. It also needs to be pointed out that in the on-state for lower $V_{\mathrm{ds}}$ the $C_{\mathrm{gd}}^{\prime}$ is stronger affected than for higher drain bias, which was anticipated by the analyses of the $g_{\mathrm{ds}}$.

Apart from all the aforementioned discussions, there is another point to consider, which is the hump in the measured $C_{\text {gd }}$ curves in the ambipolar-state and also in $C_{\text {gs }}$ in the on-state. Considering the measurement data of the p-type planner TFET (see Fig. 6.5), at $V_{\mathrm{ds}}=-0.1 \mathrm{~V}$ and for $V_{\mathrm{gs}}=-0.5 \mathrm{~V}$, a sudden growth or a knob can be seen. In [75] it is suggested that this hump is due to the transition from TAT to B2B tunneling. That means, it is assumed that in the planar TFET the TAT occurs first and leads to an increase in $C_{\mathrm{gs}}$. Thereafter, by increasing the gate bias voltage in negative direction, the B2B tunneling also starts and at this point the hump appears in the curve. The higher the drain bias, the less the hump can be seen. The reason for this can be that with the higher drain bias, or more precisely the more negative $V_{\mathrm{ds}}$ in this case, this transition occurs faster and therefore it cannot manifests itself. It can be concluded that the behavior of capacitances in TFET is obviously very responsive to the current and all elements such as traps and structure of the transistor which bring about a change in the charge carriers of the channel. 


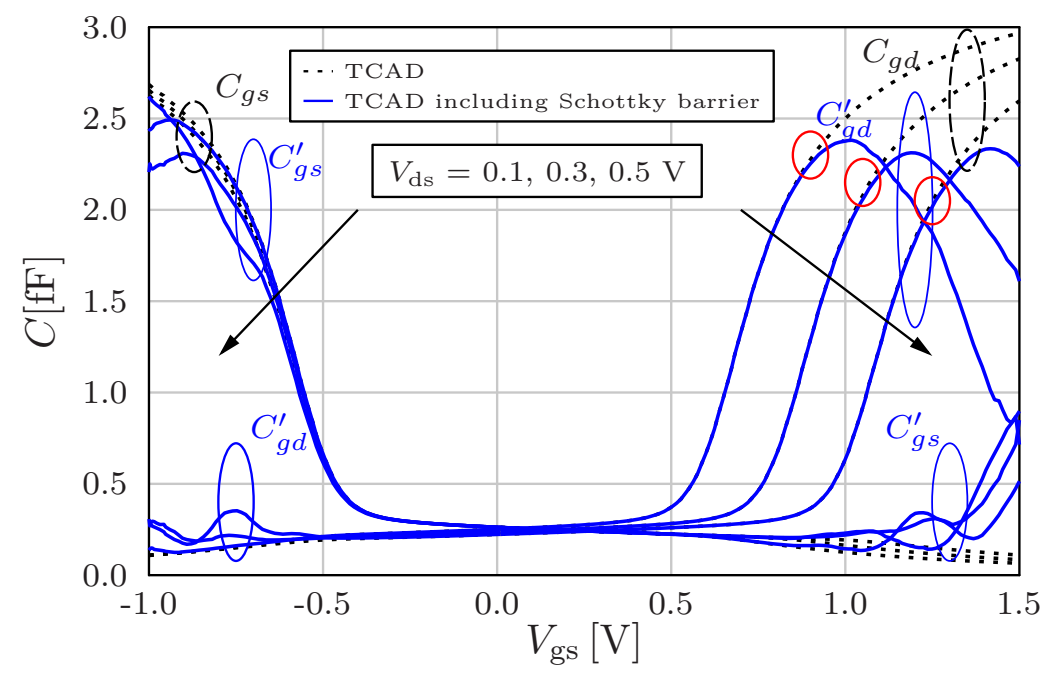

Figure 6.10: The black dotted curves represent the intrinsic capacitances of the n-type DG TFET simulated in TCAD. The blue curves illustrate the capacitances which are recalculated by including the effect of the Schottky barrier contacts effect. The red circles indicate the gate voltage at which the impact of the Schottky barrier shows itself on each curve [76].

\subsubsection{NW TFET}

The NW TFET are reported to have a higher $I_{\mathrm{on}}$, subthreshold swing below $60 \mathrm{mV} / \mathrm{dec}$ and better performance in comparison to the planar structure [80-82]. It is therefore important to check whether the compact model is also suitable for this device and can represent such a TFET.

The geometry of the vertical gate-all-around n-type Si NW TFET, that is investigated here, is illustrated in Fig. 6.11(a) and its geometrical parameters are listed in Tab. 6.2. This device is fabricated by Interuniversity Microelectronics Center (imec) Belgium. The Si substrate on which the NW is grown is heavily doped with arsenic, the channel consists of lightly n-doped $\mathrm{Si}$ and the source region of highly Boron-doped Si. Considering the cross section of this TFET depicted in Fig. 6.11(b), it can be seen that the gate overlaps the source area. According to the results reported in [83], increasing the gate-source overlap enhances the on-current of the NW TFET which is due to the control of the gate over the tunneling at the source side.

Another point of attention in this structure is the thick layer of oxide around the channel and the drain region. The purpose of implementation of this layer of oxide is to reduce the gate leakage and also suppressing the ambipolarity. This layer isolates the gate from substrate, hence, it results in a lower gate leakage as well as the gate-substrate capacitance [83]. Furthermore, due to the gate underlap region at the drain side, the tunneling on this side and consequently the ambipolar-current is suppressed. Further information about the steps and details of the fabrication process are given in [83]. 


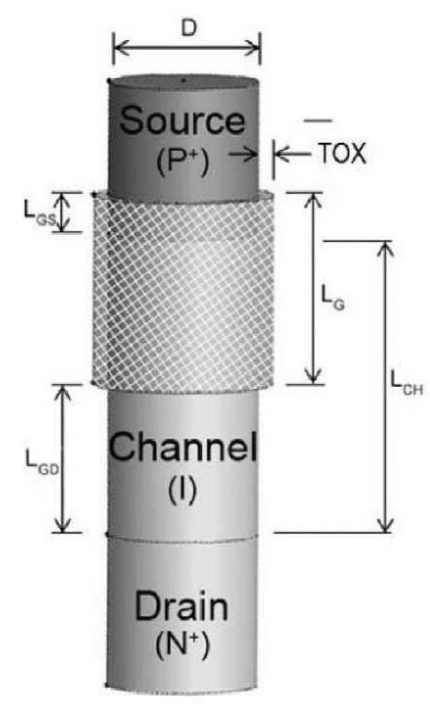

(a)

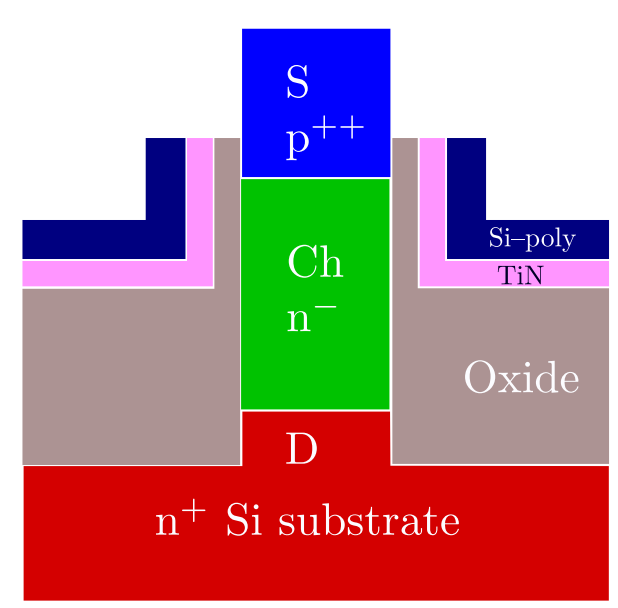

(b)

Figure 6.11: (a) The schematic of the fabricated n-type Si NW TFET and (b) its cross section $[83,84]$.

Table 6.2: The structural parameters of the n-type Si NW TFET.

\begin{tabular}{|c|c|c|c|}
\hline Parameter & Value & Parameter & Value \\
\hline$L_{\mathrm{CH}}$ & $220 \mathrm{~nm}$ & $L_{\mathrm{G}}$ & $150 \mathrm{~nm}$ \\
\hline$L_{\mathrm{GS}}$ & $30 \mathrm{~nm}$ & $L_{\mathrm{GD}}$ & $100 \mathrm{~nm}$ \\
\hline$D$ & $200 \mathrm{~nm}$ & $N_{\mathrm{CH}}$ & $10^{15} \mathrm{~cm}^{-3}$ \\
\hline$N_{\mathrm{s}}$ & $10^{20} \mathrm{~cm}^{-3}$ & $N_{\mathrm{d}}$ & $10^{19} \mathrm{~cm}^{-3}$ \\
\hline$T_{\mathrm{OX}}$ & $4 \mathrm{~nm}$ & OX. Mat & $1 \mathrm{~nm} \mathrm{SiO} 2+3 \mathrm{~nm} \mathrm{HfO}_{2}$ \\
\hline
\end{tabular}

The measurement data associated with the NW TFET depicted in Fig. 6.11 are provided by LSI/PSI/USP, University of Sao Paulo, Brazil and are partially presented in [84, 85]. The transfer characteristic curves are obtained doing the measurements for a device with 400 parallel NW TFETs at room temperature [84]. The capacitance measurements are carried out for a device with $4050 \mathrm{NW}$ TFETs connected in parallel at a frequency of $10 \mathrm{kHz}$, at room temperature, $V_{\mathrm{ds}}=0 \mathrm{~V}$ and grounded source terminal [85].

Taking into account the fact that the model is originally designed for a DG TFET, and considering the cylindrical shape of the NW and its gate-source overlap, the compact model must first be tailored to this structure in terms of the geometrical parameters of the device and fitting factors. The fitting parameters need to be chosen appropriately so that the model shows a good agreement with the measured data, whereby these parameters should be reasonable and physically justifiable. In this regard, the equivalent geometric parameters are implemented in 
the compact model to achieve a perfect match with the measurements.

In the compact model the equivalent oxide thickness (EOT) is assigned to $t_{\mathrm{ox}}$. This value shows, if instead of the material with larger dielectric constant $(\kappa)$ such as $\mathrm{HfO}_{2}, \mathrm{SiO}_{2}$ is used, how thick this layer is supposed to be to achieve a similar performance. The EOT is given as $[11,86]$ :

$$
E O T=t_{\mathrm{hk}} \cdot \frac{\kappa_{\mathrm{SiO}_{2}}}{\kappa_{h k}},
$$

where $t_{\mathrm{hk}}$ is the thickness of the oxide with high permittivity, $\kappa_{\mathrm{SiO}_{2}}$ is the dielectric constant of $\mathrm{SiO}_{2}$ and $\kappa_{\mathrm{hk}}$ characterizes the dielectric constant of the high- $\kappa$ oxide. Considering Tab. 6.2,

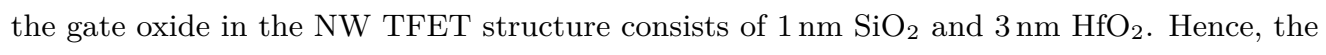
EOT is calculated as follows:

$$
E O T=t_{\mathrm{HfO}_{2}} \cdot \frac{\kappa_{\mathrm{SiO}_{2}}}{\kappa_{\mathrm{HfO}_{2}}}+t_{\mathrm{SiO}_{2}}
$$

where $\kappa_{\mathrm{HfO}_{2}}=25$ and $\kappa_{\mathrm{SiO}_{2}}=3.9$. So, the calculated EOT for this structure device is equal to $1.4 \mathrm{~nm}$.

According to the definition of the channel length in the TFET compact model, it is assumed that $l_{\mathrm{ch}}$ of the NW TFET is equal to $220 \mathrm{~nm}$ ( $L_{\mathrm{CH}}$ in Fig. 6.11(a)). However, the studies performed on the DG MOSFET compact model show that the equivalent $l_{\mathrm{ch}}$ should be larger than the corresponding value in the structure to allow simulations of the gate-all-around MOSFET with the existing DG model [87]. Based on it, here $l_{\mathrm{ch}}$ is chosen to be equal to $250 \mathrm{~nm}$. Regarding the other structural parameters in the compact model, both $t_{\mathrm{ch}}$ and $w_{\mathrm{ch}}$ are considered to be equal to $D=200 \mathrm{~nm}$. In order to compensate the effect of the gate-source overlap, a parallel capacitance to $C_{\text {eff }}$ is considered in the model which serves for an increment in the maximum value of the intrinsic capacitances. Since the measurements are performed for devices with multiple NW TFETs, the data is in each case divided by the number of NWs and then the compact model is fitted to the data corresponding to a single NW TFET.

After setting all device parameters in the compact model and choosing appropriate fitting parameter, the measured $I_{\mathrm{ds}}$ as a function of $V_{\mathrm{gs}}$ at three different $V_{\mathrm{ds}}$ is compared to the compact model and the results are illustrated in Fig. 6.12. Also the measured $C_{\mathrm{gs}}, C_{\mathrm{gd}}$ and $C_{\mathrm{gg}}$ are compared to those obtained by the compact model. The results are shown in Fig. 6.13. It must be mentioned that here the effect of the Schottky barrier is neglected in the model. However, as it can be seen in both Fig. 6.12 and Fig. 6.13, the model is in a very good agreement with the measurements.

In this step, it has become apparent that the model can properly and effectively describe the behavior of the intrinsic capacitances in a TFET considered as a single component with respect to the simulations and measured data. So, the compact model is then implemented in Verilog-A language to allow circuit simulations. In the next chapter, the steps which are taken to design some simple circuits and to investigate the efficiency of the compact model in terms of circuit simulation, are presented and discussed in detail. 


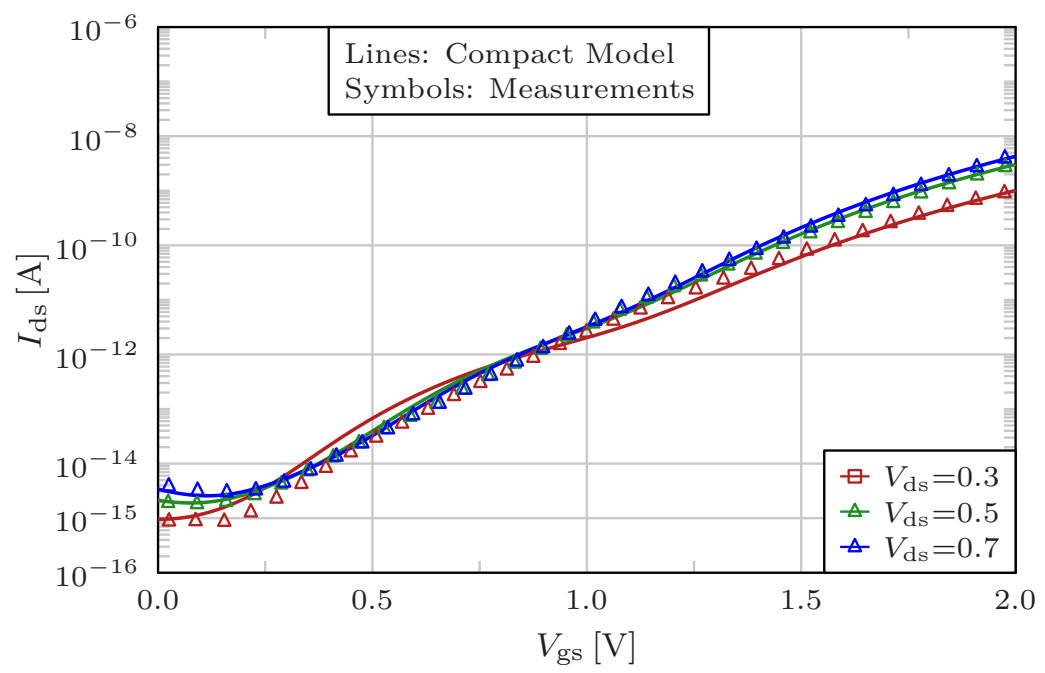

Figure 6.12: The measured $I_{\mathrm{ds}}$ of the n-type NW TFET for varying $V_{\mathrm{gs}}$ is compared to the results of the compact model at different $V_{\mathrm{ds}}$.

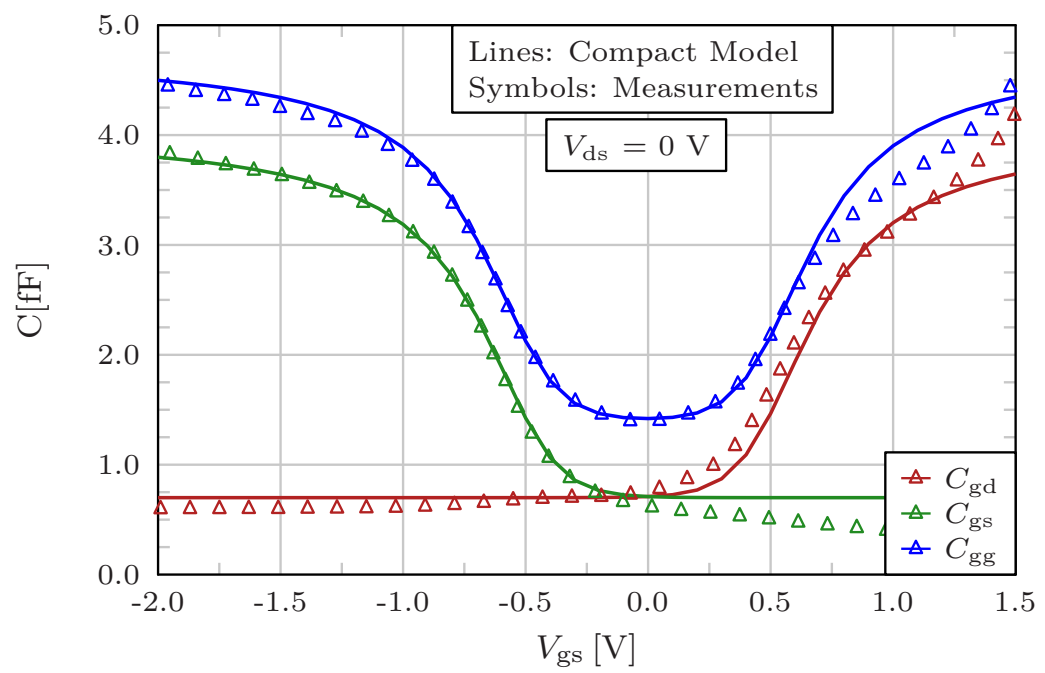

Figure 6.13: The measured capacitances of the $n$-type NW TFET for varying $V_{\text {gs }}$ at $V_{\mathrm{ds}}=0 \mathrm{~V}$ are compared to the corresponding capacitances obtained by the compact model. 


\section{CHAPTER 7}

\section{Circuit Simulation}

In the following, the model is used to perform circuit simulations. In this regard, the model is firstly implemented in Verilog-A language. Then it is used to design p-type and n-type TFET and by means of them some simple digital circuits, such as inverter and ring-oscillator, are designed. Thereafter, the outputs of these circuits are compared and presented by considering different device parameters.

This chapter is divided into two main parts. In Sec. 7.1 circuit simulations are performed for those circuits which are designed by means of DG TFET and the effect of device parameters on results are studied. The Sec. 7.2 presents the results of simulations in which the circuits are based on SG line tunneling TFETs.

\subsection{DG TFET-Based Circuits}

It is important to check if the model is robust enough for circuit simulations and also inspect how it responses by varying the device parameters. In this context, after the DC and the intrinsic capacitance models are brought together and linked, some parameters are chosen to be changed and their impact on the static and transient performance of the TFET-based circuits is considered.

The structure which is focused on in this part is a DG TFET with $l_{\mathrm{ch}}=22 \mathrm{~nm}$ (see Fig. 4.1) and for the sake of the simplicity, the effect of the Schottky barrier contact is neglected in the model. The device parameters which are selected to be examined are the trap density $\left(N_{\mathrm{t}}\right)$ at the tunneling junction, $N_{\mathrm{d}}$ and $l_{\mathrm{ch}}$. Also here the compact model is in the first place compared with the TCAD simulation results of one transistor.

To begin with, the transfer characteristics of the n-type TFET in four cases are compared with corresponding simulations in TCAD Sentaurus in Fig. 7.1. Reducing $N_{\mathrm{d}}$ leads to a suppression in ambipolarity and decreasing $N_{\mathrm{t}}$ results in lower current in off-state of the device. Considering the first case, which is shown in red, both $N_{\mathrm{d}}$ and $N_{\mathrm{t}}$ are reduced, it means that the effect of ambipolarity and traps are suppressed. The green curves show the setup in which 
only traps are reduced, the blue ones refer to the case that just ambipolarity is suppressed and for the purple curves both effects are remained at their maximum. It can be seen that for all these cases the compact model shows a good agreement with the results obtained from TCAD simulations. The same steps are also taken to validate the compact model in the case of the p-type TFET.

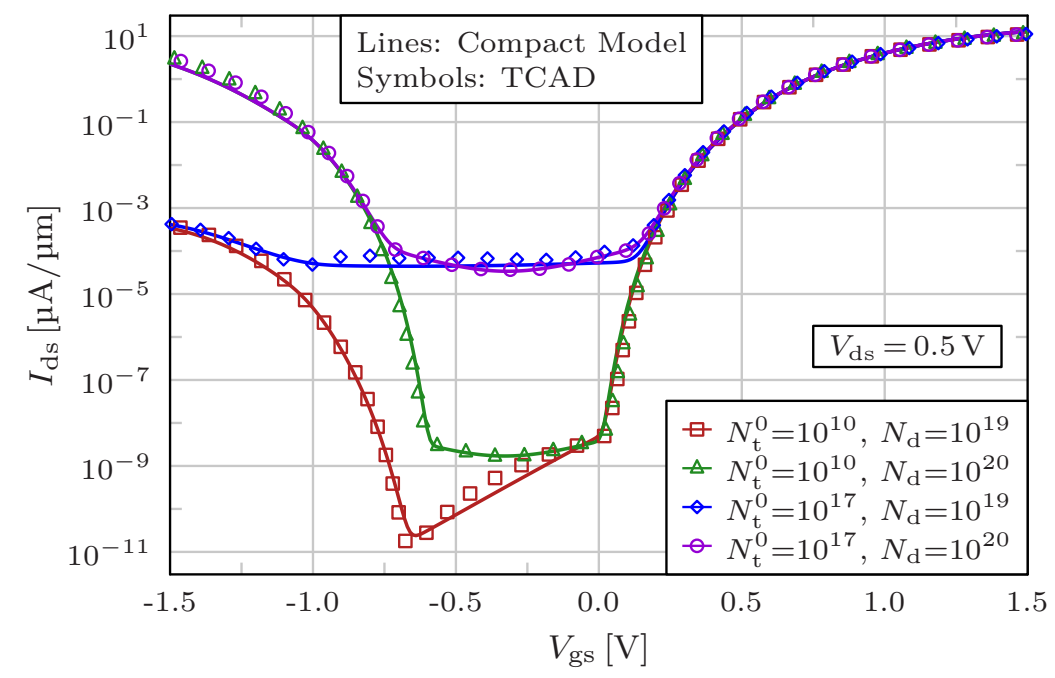

Figure 7.1: Current transfer characteristic of an n-type DG TFET in four different combinations of $N_{\mathrm{d}}$ and $N_{\mathrm{t}}$. In each case the result of the compact model is compared with TCAD simulation output. The dimensions of the device are defined as following: $l_{\mathrm{ch}}=22 \mathrm{~nm}, t_{\mathrm{ch}}=10 \mathrm{~nm}$, $t_{\mathrm{ox}}=2 \mathrm{~nm}$ of $\mathrm{HfO}_{2}, w_{\mathrm{ch}}=1 \mu \mathrm{m}$, the source is Boron doped with $N_{\mathrm{s}}=10^{20} \mathrm{~cm}^{-3}$ and in the drain region Phosphorus with the given doping concentrations is implemented [88].

After verification of the DC model for varying $N_{\mathrm{t}}$ and $N_{\mathrm{d}}$ and for the both n- and p-type transistors, the intrinsic capacitances model is examined in comparison to the TCAD simulations as well. In Fig. 7.2 capacitances of a p-type device are illustrated. For $V_{\mathrm{gs}}<0$ the transistor is in its on-state, hence the mobile charges in the channel cause an increase in $C_{\mathrm{gd}}$. For $V_{\mathrm{gs}}>0$ the p-type TFET is in ambipolar-state and therefore $C_{\text {gs }}$ takes over. Considering the green and purple curves in Fig. 7.2 it can be seen that in these setups $N_{\mathrm{d}}=10^{20} \mathrm{~cm}^{-3}$ but $N_{\mathrm{t}}$ is different. However, the change in $N_{\mathrm{t}}$ has a minimal impact on the $C_{\mathrm{gs}}$ and almost no effect on $C_{\mathrm{gd}}$. By comparing these two curves with the red and blue ones, in which $N_{\mathrm{d}}=10^{19} \mathrm{~cm}^{-3}$ and $N_{\mathrm{t}}$ is equal to $10^{10} \mathrm{~cm}^{-2}$ and $10^{17} \mathrm{~cm}^{-2}$ respectively, it can be said that increasing $N_{\mathrm{d}}$ results into a higher $C_{\mathrm{gs}}$. Generally speaking, in all these four cases the $C_{\mathrm{gd}}$ is not significantly affected and $C_{\mathrm{gs}}$ is only influenced by the change in the value of $N_{\mathrm{d}}$. The intrinsic capacitance model, depicted with solid black lines in Fig. 7.2, fulfills the expectations and fits the TCAD simulations.

After calibrating the compact model by means of TCAD simulation for both $\mathrm{n}$-type and p-type devices, it is implemented in the Cadence electronic design automation software [89]. 


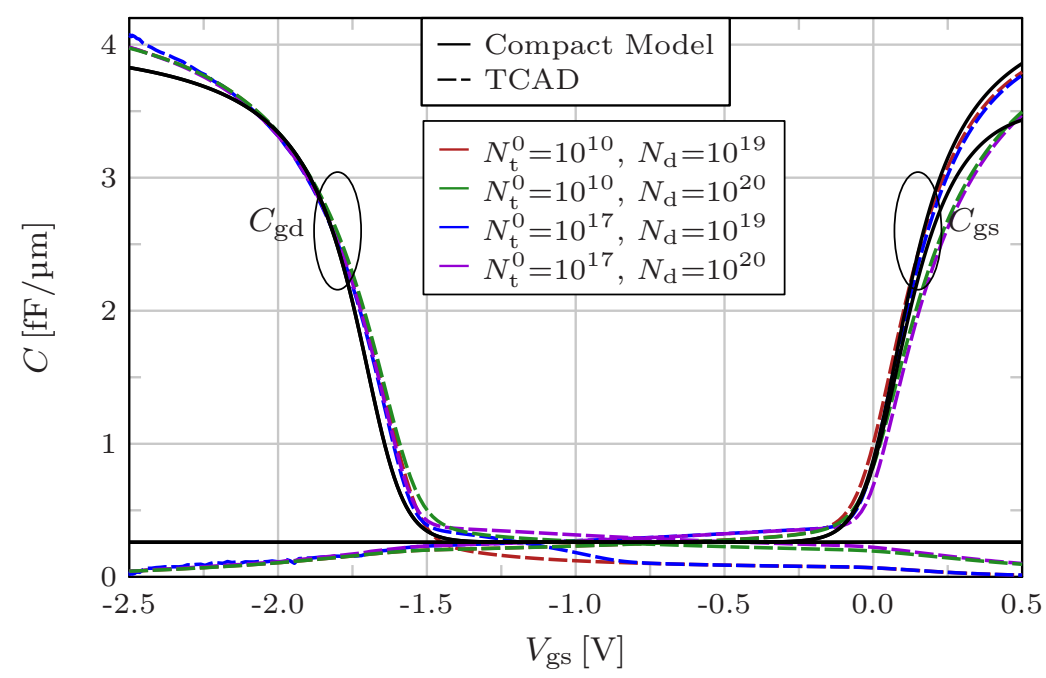

Figure 7.2: The intrinsic capacitances of a p-type DG TFET in four different combinations of $N_{\mathrm{d}}$ and $N_{\mathrm{t}}$. In each case the result of the compact model is compared with TCAD simulation. The dimensions of the device are defined as following: $l_{\mathrm{ch}}=22 \mathrm{~nm}, t_{\mathrm{ch}}=10 \mathrm{~nm}, t_{\mathrm{ox}}=2 \mathrm{~nm}$ of $\mathrm{HfO}_{2}, w_{\mathrm{ch}}=1 \mu \mathrm{m}$, the source is Phosphorus doped with $N_{\mathrm{s}}=10^{20} \mathrm{~cm}^{-3}$ and in the drain region Boron is used as the dopant [88].

Although the aim was focusing on the aforementioned device parameters, in early stages of experimenting with the model it has got apparent that shifting the $V_{\mathrm{fb}}$ significantly affects the performance of the TFET-based circuits. In the case that there is no charge existing in the oxide or at the oxide-semiconductor interface, $V_{\mathrm{fb}}$ is depending on the work function of the gate metal and the semiconductor. Which means, it is not a fitting parameter and rather supposed to be calculated from band diagram. However, as it is mentioned here the aim is to examine the behavior of the TFET-based circuits considering different device parameters. Hence, after some experiments two main setups are chosen to be used for the simulations, namely the low-power mode (LP) and high-performance mode (HP). In Fig. 7.3 the tranfer curves of both n- and p-type TFETs in each of these two modes are illustrated. It can be seen that shifting $V_{\mathrm{fb}}$ by $50 \mathrm{mV}$ results in a lower $I_{\mathrm{ds}}$ for small $V_{\mathrm{gs}}$, which is in fact the reason this mode is called LP. On the other hand, shifting $V_{\mathrm{fb}}$ by $-200 \mathrm{mV}$ leads to a higher $I_{\mathrm{ds}}$, which means the performance of the transistor for lower $V_{\mathrm{gs}}$ is improved and therefore this setup is called HP. Considering Fig. 7.3 it can also be extracted that the maximum slope of the transfer curve in LP mode is around $26 \mathrm{mV} / \mathrm{dec}$, while in the HP mode this value is almost equal to $70 \mathrm{mV} /$ dec. This very moderate slope in HP mode is due to the shift in the $V_{\mathrm{fb}}$ and it can be argued that it is far worse than what is expected in the TFET. But it needs to be noted that the main goal of these simulations is to investigate the performance of the circuits for the case in which $I_{\mathrm{ds}}$ is higher. Hence, such a trade-off to improve the current, yet by using a simple $\mathrm{Si}$ DG TFET structure, would have been inevitable at this point [88]. In the light of these two 
setups, an inverter circuit is designed using n- and p-type TFETs in Cadence Virtuoso [89], which is afterwards used to compose a 3-stage ring-oscillator (see Fig. 7.4).

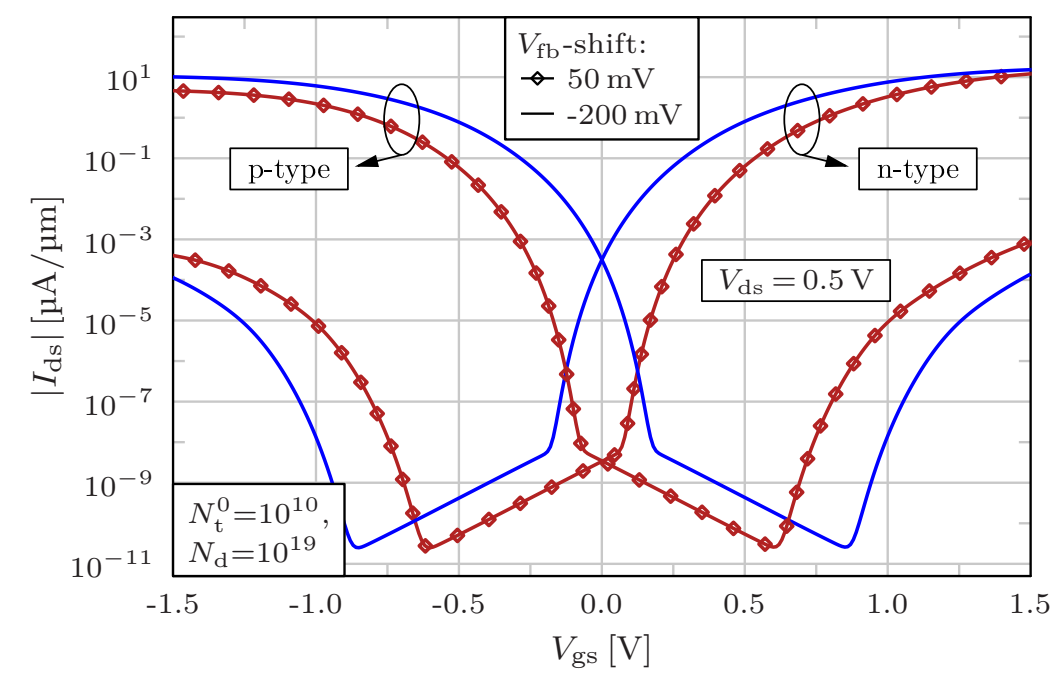

Figure 7.3: Transfer characteristic of the $n-$ and $p$-type DG TFETs are shown for the LP mode with $V_{\mathrm{fb}}-$ shift $=50 \mathrm{mV}$ and $\mathrm{HP}$ mode with $V_{\mathrm{fb}}-$ shift $=-200 \mathrm{mV}$ [88].

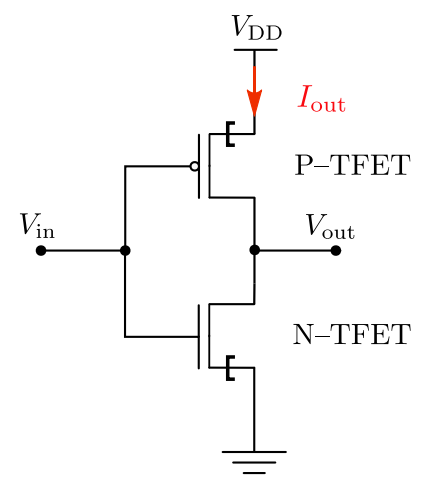

(a)

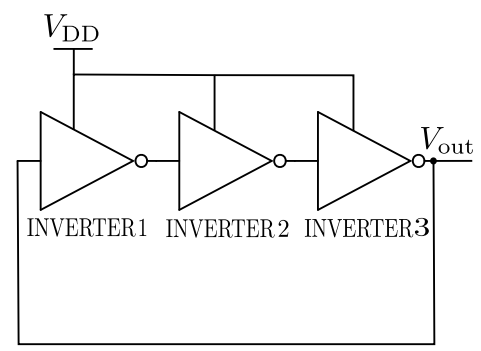

(b)

Figure 7.4: (a) Schematic of the TFET-based inverter circuit simulated in Cadence. (b) 3stage ring-oscillator using the TFET-based inverters. $V_{\mathrm{DD}}$ refers to the input voltage, $V_{\mathrm{in}}$ is the input voltage of the inverter, $V_{\text {out }}$ represents the output voltage and $I_{\text {out }}$ refers to the output current [88]. 
To begin with, it must be specified that the supply voltage of inverters ( $\left.V_{\mathrm{DD}}\right)$ (see fig. 7.4), in all following simulations is set to $0.5 \mathrm{~V}$ except it is mentioned. In Fig. 7.5(a) the voltage transfer curves (VTC) of TFET-based inverter are shown in HP mode and for three cases regarding the suppression of $N_{\mathrm{t}}$ and $N_{\mathrm{d}}$. Furthermore, on the left axis, the output current $\left(I_{\text {out }}\right)$ which is indicated in Fig. 7.4(a), is illustrated for all these setups. For the input voltage $V_{\text {in }}=0 \mathrm{~V}$ the p-type TFET is in its on-state. So, it is expected that $V_{\mathrm{DD}}$ with a low resistance is connected to the output of the inverter and hence $V_{\text {out }}=0.5 \mathrm{~V}$. In other words, when the upper transistor, the p-type TFET, is in on-state it can be treated as a low resistance which leads to $V_{\text {out }}=V_{\mathrm{DD}}$. When $V_{\text {in }}$ rises, eventually the gate bias of the transistors increases and as a result the n-type TFET begins to switch on and in contrast the p-type device goes off. It can be seen in Fig. 7.5(a) that in the short moment that both transistors are on $I_{\text {out }}$ reaches its maximum which is due to the created path between $V_{\mathrm{DD}}$ and the ground [88]. Further increment in $V_{\text {in }}$ leads to a decrement in the output voltage and finally $V_{\text {out }}=0 \mathrm{~V}$, which shows the p-type TFET is in the off-state. Considering the changes in $N_{\mathrm{t}}$ and $N_{\mathrm{d}}$, it can be seen that all the curves look very similar, which means the traps and ambipolarity do not have a significant influence on the switching behavior of the HP-TFET Inverter.

In Fig. 7.5(b) VTC of the TFET in HP mode is compared with the one in LP mode. Here by contrast, a notable difference between these two setups can be observed. The VTC curve in LP mode has a steeper slope than in HP mode, which means the LP-TFET has a higher gain. Taking the VTC curves into account, the DC gain of the LP-TFET is approximately equal to 14, and in HP mode this value is close to 3.5. In addition, as expected, it can be seen that the HP-TFET has a higher value of $I_{\text {out }}[88]$.

Despite the fact that the suppression of the traps and the ambipolarity of the TFET influences its electrical properties, no significant impact on the results of the inverter simulations can be seen. But by considering Fig. 7.5 and looking back at Fig. 7.1, one can see that in the defined range of $V_{\text {in }}$ for VTC in both HP and LP mode, neither the effect of TAT on the subthreshold swing nor the effect of ambipolarity suppression is attained. That means, for the applied gate voltage, degradation of the subthreshold swing due to the changes in $N_{\mathrm{t}}$ is not manifesting itself and it is also not reached in the ambipolar-state. The current which is dominating in transient simulation is the charging current. So, for example, the effect of $N_{\mathrm{t}}$ only becomes visible when the circuit is in a steady state, in which the off-current is influenced by traps [88]. Hence, hereafter the focus is moved to the $V_{\mathrm{fb}}-$ shift.

To perform transient simulations for the TFET-based inverter, a rectangular pulse is given as the input of the inverter. In Fig. 7.6 the response of the inverter to the indicated pulse in HP mode is shown in blue and for the LP mode in red. When the input transits from 0 to its high value, the inverter switches off. Before the current tends towards 0 , it shows an even higher positive peak during the rise-time of the input pulse and then sinks to reach its low value. While the input drops to its minimum, the output first reaches its negative peak and then rises to its high value. In other words, at the rising edges of the input signal the output shows an overshoot and at the falling edges an undershoot, which is due to the capacitances in 
the TFET compact model. Starting from $V_{\text {in }}=0 \mathrm{~V}$, the p-type TFET is on and the holes are accumulated in the channel. In the moment that it is switching to its high level, the charge carriers in the channel of both transistors proceed as the Miller capacitance. Hence, by further increment in the value of $V_{\text {in }}$, just before that the n-TFET is in on-state, the output signal overshoots and goes beyond $V_{\mathrm{DD}}[88,90]$. When the n-type TFET is turned on and starts to conduct the current flow, the holes in the channel can move through the $n-i-p$ junction of the p-type TFET which is forward-biased. In this state the negative charge carriers tunnel through the barrier on the source side of the n-type device and accumulate in the channel. As the result, this charging effect reduces the rate at which the output voltage slows down [88].

Considering the HP and LP setups in Fig. 7.6, it can be seen that the overshoot and undershoots have a smaller peak in HP mode, which is due to the fact that in this case the transistors are earlier turned on. In other words, since in HP mode TFETs are supplied by a higher current, the charge carriers get faster accumulated in the channel of the device which is supposed to get on.

At $V_{\text {in }}=0.5 \mathrm{~V}$, the capacitances in p-type TFET are discharged and the channel of the $\mathrm{n}$-type device is accumulated with electrons. When the input pulse starts to drop, at this moment the n-TFET is forward-biased. Hence, its channel charge carriers get released to the ground and an undershoot in $V_{\text {out }}$ appears. Furthermore, in this case holes tunnel through the barrier on the source junction of the p-TFET into its channel and limit the $I_{\text {out }}$ as well as the rising time of the $V_{\text {out }}[88]$. 


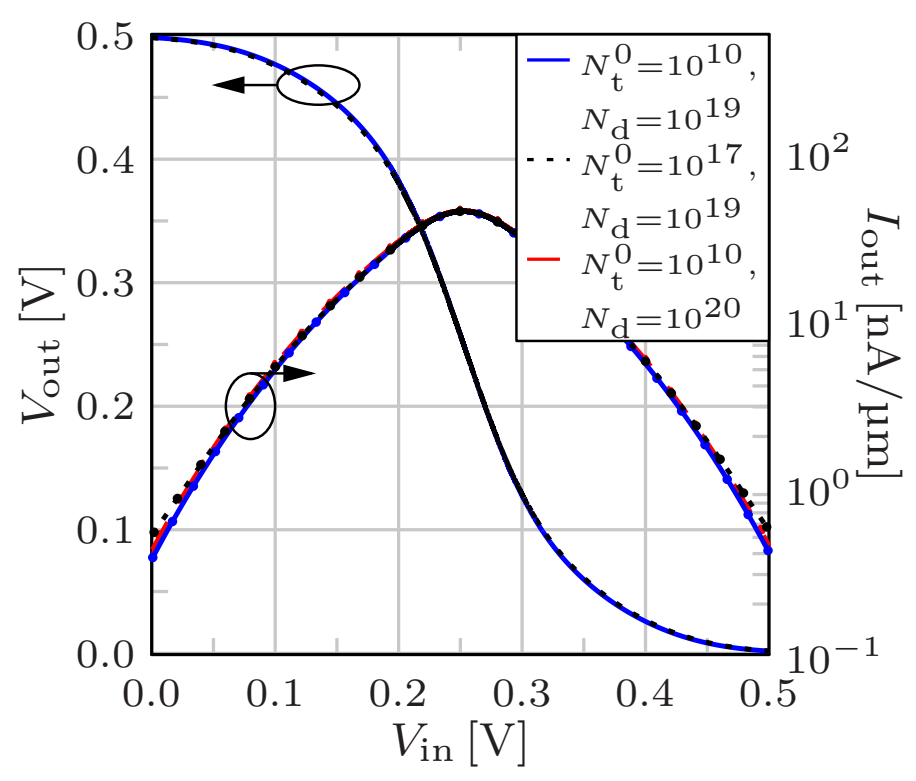

(a)

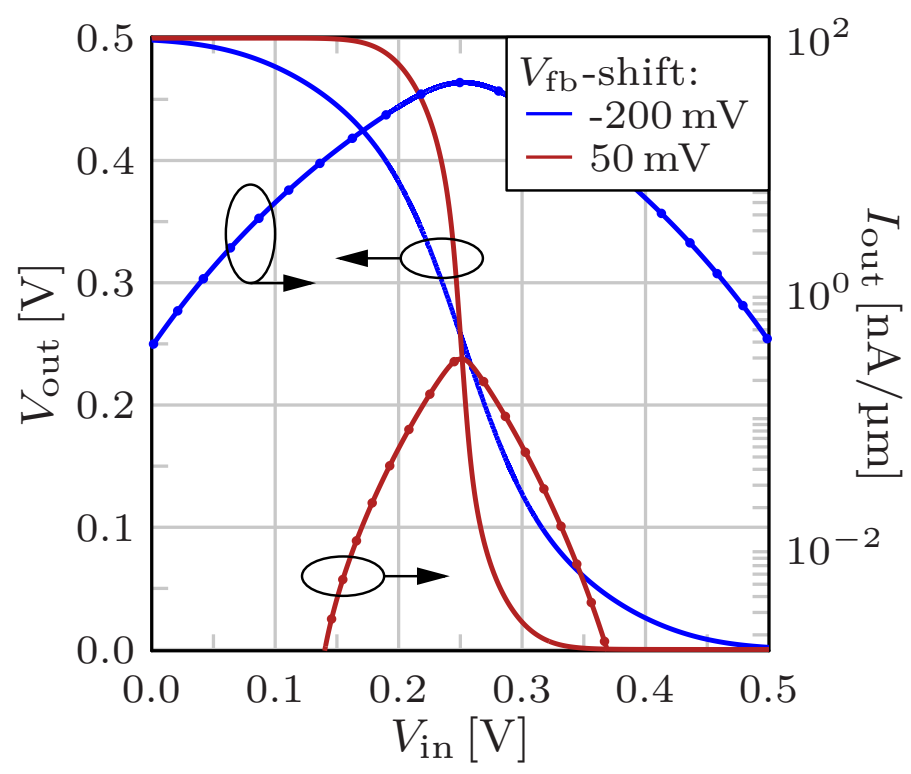

(b)

Figure 7.5: In (a) VTC and current curves of the TFET-based inverter in HP mode for three different cases are presented. In (b) VTC and $I_{\text {out }}$ of the HP-TFET inverter with $V_{\mathrm{fb}}-$ shift $=-200 \mathrm{mV}$ for $N_{\mathrm{t}}=10^{10} \mathrm{~cm}^{-2}$ and $N_{\mathrm{d}}=10^{19} \mathrm{~cm}^{-3}$ are compared with those of the LP inverter in which $V_{\mathrm{fb}}-$ shift $=50 \mathrm{mV}$ [88]. 


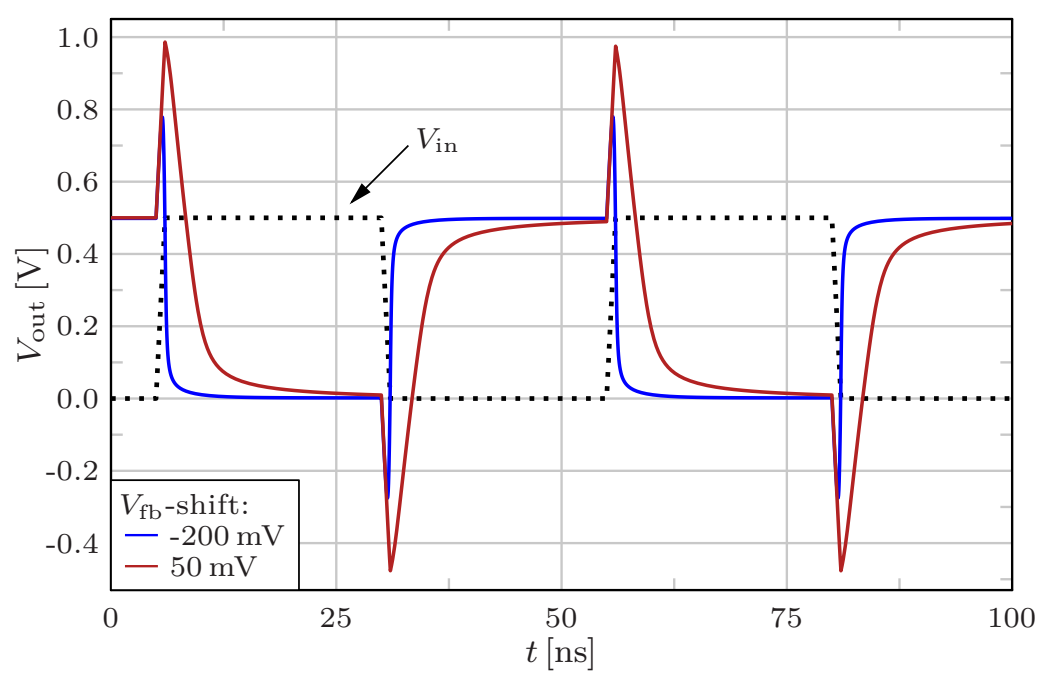

Figure 7.6: The effect of the $V_{\mathrm{fb}}$-shift on the output waveform of the TFET-base inverter is investigated. To perform the transient simulations a trapezoids with very steep edges is considered as the input of the inverter. At the rising edge $V_{\text {out }}$ shows a overshoot and at the falling edge it shows an undershoot which is due to the intrinsic capacitances [88].

After analyzing the behavior of the TFET-based inverter, a 3-stage ring-oscillator is simulated. In Fig. 7.7 the output voltage of this oscillator as a function of time is presented. As it is explained in previous paragraphs, it can be said that in HP mode due to a higher on-current charging and discharging of intrinsic capacitances occurs faster than in LP mode. Thus, it is expected that the 3-stage ring-oscillator in HP shows a smaller delay comparing to the LP case and it is exactly what is illustrated in Fig. 7.7 [88].

In order to investigate the effect of the $l_{\mathrm{ch}}$, the ring-oscillator simulations are repeated for different channel lengths of TFETs. With the help of these simulations, first the oscillation periodic represented by $T$ in Fig. 7.7 is calculated and then from this the intrinsic delay of a single inverter $(\tau)$ is derived as follows:

$$
\tau=\frac{T}{2 n}
$$

where $n$ refers to the number of stages in the ring-oscillator circuit which in this case is equal to three.

In Fig. 7.8 the calculated $\tau$ from ring-oscillator simulations for $l_{\mathrm{ch}}=22,45,65,90,180$ and $250 \mathrm{~nm}$ in HP and LP cases are depicted with symbols. Generally speaking, it can be seen that in HP mode the ring-oscillator shows a smaller delay than in the LP mode and in both setups by increasing $l_{\mathrm{ch}}$ also the delay increases. More precisely, for $l_{\mathrm{ch}}=22 \mathrm{~nm}$ the inverter delay in HP mode is equal to $6.7 \mathrm{~ns}$ and this value raises to $68 \mathrm{~ns}$ for $l_{\mathrm{ch}}=250 \mathrm{~nm}$. In LP mode these corresponding delay values are $115 \mathrm{~ns}$ and $1.2 \mu \mathrm{s}$, respectively. Moreover, according to the 


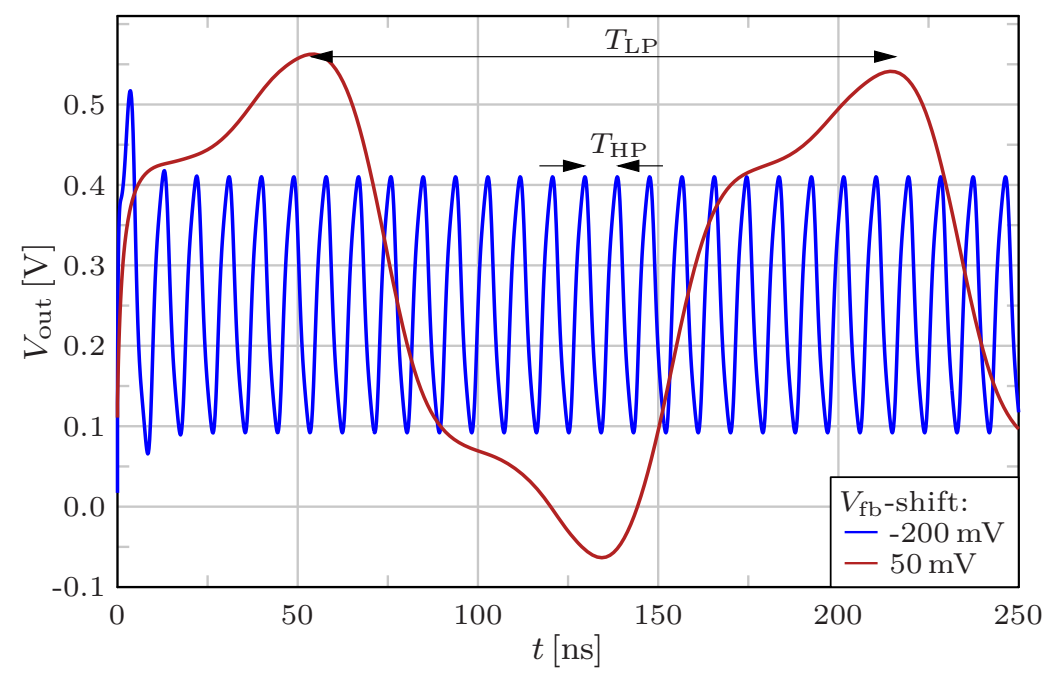

Figure 7.7: $V_{\text {out }}$ of the 3-stage ring-oscillator. $T_{\mathrm{LP}}$ and $T_{\mathrm{HP}}$ refer to the period of the oscillation in LP and HP case, respectively. In LP mode, which is shown in red, the delay is bigger than in the HP mode illustrated in blue. In both setups $V_{\mathrm{DD}}=0.5 \mathrm{~V}$ [88].

simulations the average output current of each inverter is remained almost independent from $l_{\mathrm{ch}}$, approximately $55 \mathrm{nA} / \mu \mathrm{m}$ for the $\mathrm{HP}$ case and $5 \mathrm{nA} / \mu \mathrm{m}$ for the LP mode. Additionally to the calculated inverter delay from ring-oscillator simulations, it is also estimated based on the following well-known analytical expression:

$$
\tau=\frac{C \cdot V}{I}
$$

In this Eq. it is considered that $C=C_{\mathrm{ox}}^{\prime} w_{\mathrm{ch}} l_{\mathrm{ch}}+2 C_{\text {ext }}$ and $C_{\text {ext }}$ refers to the extrinsic capacitance effect on the intrinsic part of the TFETs. The current $I$ is set as a fitting parameter in this case, which is equal to $0.17 \mu \mathrm{A} / \mu \mathrm{m}$ for the HP mode and $10 \mathrm{nA} / \mu \mathrm{m}$ for the LP mode. In consideration of Fig. 7.3 these values are chosen in a way that they are smaller than the on-current at $\left|V_{\mathrm{gs}}\right|=0.5 \mathrm{~V}$, which is however not reached in the range that inverters sweep [88].

In the light of Fig. 7.8 one can conclude that the intrinsic delay of a TFET-based inverter is proportional to the $l_{\mathrm{ch}}$ of its transistors. It is derived by taking into account the Eq. (7.2) and considering that the capacitances in TFET are a function of $l_{\mathrm{ch}}$ but not of the current. In other words, in a TFET the tunneling barriers are the bottleneck for the current, hence varying $l_{\text {ch }}$ does not significantly affect the current [35]. For comparison, the corresponding curve of a CMOS inverter in the $32-\mathrm{nm}$ node with $V_{\mathrm{DD}}=0.8 \mathrm{~V}$ is also shown here. That is to say, based on the International Technology Roadmap for Semiconductors (ITRS) reports [91] the values of on-current and intrinsic inverter delay for the supply voltage of $0.8 \mathrm{~V}$ and $l_{\mathrm{ch}}=32 \mathrm{~nm}$ are obtained as: $I_{\mathrm{on}}=1.7 \mu \mathrm{A} / \mu \mathrm{m}$ and $\tau=2.1 \mathrm{ps}$. Then, on the basis of these informations and 


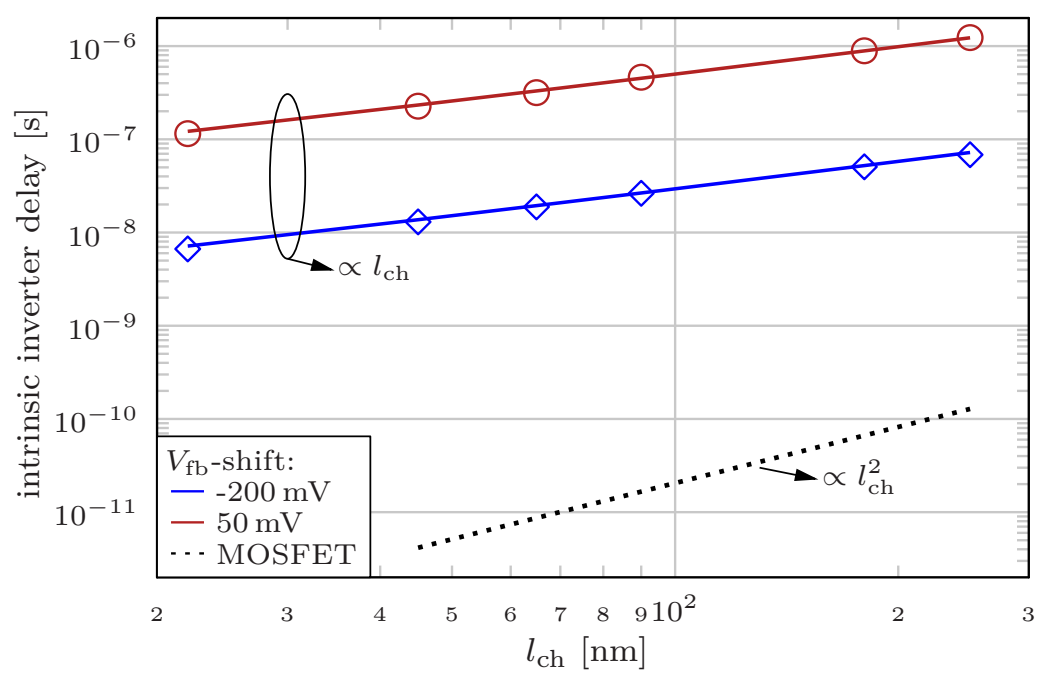

Figure 7.8: Intrinsic inverter delay extracted from simulations of TFET-based ring-oscillator circuit for $l_{\mathrm{ch}}=22,45,65,90,180$ and $250 \mathrm{~nm}$ are shown with symbols. The solid lines represent the theoretical relation between $\tau$ and $l_{\mathrm{ch}}$. The dotted line shows the corresponding correlation for the CMOS-inverter [88].

with the help of the Eq. (7.2), first the capacitance and then the CMOS inverter delay for different channel lengths are approximated and depicted in dotted line in Fig. 7.8. Since in a CMOS the current is inversely proportional to $l_{\mathrm{ch}}$ and in addition capacitance is directly corresponding to $l_{\mathrm{ch}}$, in this case $\tau \propto l_{\mathrm{ch}}^{2}[88]$.

All in all, these results demonstrate that in order to improve the dynamic performance of TFETs, their on-current must be increased, and the question arises as to how such an improvement can be achieved [76]. Studies have shown that innovative architectures of TFETs, such as those using line tunneling and hetero-structures, increase the potential to achieve a higher $I_{\text {on }}$ [92]. Hence, in the following the effect of line-tunneling is implemented in the compact model and inspected.

\subsection{SG Line Tunneling TFET-Based Circuits}

As it is already explained in Sec. 4.2, line tunneling allows for a larger tunneling region which means the source can provide the channel with more charge carriers and it leads to a higher on-current. Furthermore, since the tunneling region on the drain side stays unchanged as in the point tunneling structure, this concept can additionally reduce the ambipolarity. The aim here is to estimate the potential impact of the improved on-current on the static and dynamic performance of the TFET and circuits based on such a TFET. Hence, in the light of the scalability and flexibility of the compact model, first it is adjusted to a SG TFET, then optimized for the line tunneling case and in the end the model is used to simulate some digital 
circuits.

To investigate the accuracy of the model for SG TFET, the structure shown in Fig. 7.9 is simulated in TCAD Sentaurus and the resulting electrical properties are compared with those obtained by means of the compact model. Then to tailor the model to an ideal line tunneling TFET some assumptions are applied. It is considered that the on-current in a line tunneling device is factor $\beta$ greater than in a point tunneling TFET and in addition, to reduce the ambipolarity $N_{\mathrm{d}}$ is set to $10^{19}$. In order to implement this effect in the model, in the point tunneling case $\beta=1$ and for the line tunneling TFET the factor $\beta=1000$. In Fig. 7.10 the transfer characteristic of both $n$-type and $\mathrm{p}$-type TFETs in point tunneling case as well as the line tunneling case are presented. It can be seen that by increasing $\beta$ the curves are simply shifted and the device shows a higher on-current.

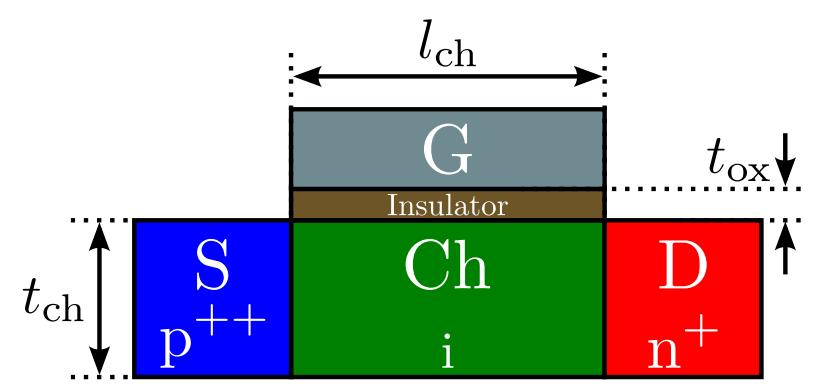

Figure 7.9: $2 \mathrm{D}$ sketch of the $\mathrm{n}$-type SG point tunneling TFET. The model verification for the $\mathrm{SG}$ device is performed in comparison with the results of the TCAD simulations executed for this structure. The structural parameters are as follows: $l_{\mathrm{ch}}=22 \mathrm{~nm}, t_{\mathrm{ch}}=10 \mathrm{~nm}, t_{\mathrm{ox}}=2 \mathrm{~nm}$ of $\mathrm{HfO}_{2}, N_{\mathrm{S}}=10^{20} \mathrm{~cm}^{-3}, N_{\mathrm{d}}=10^{19} \mathrm{~cm}^{-3}$ and the channel is intrinsic [62].

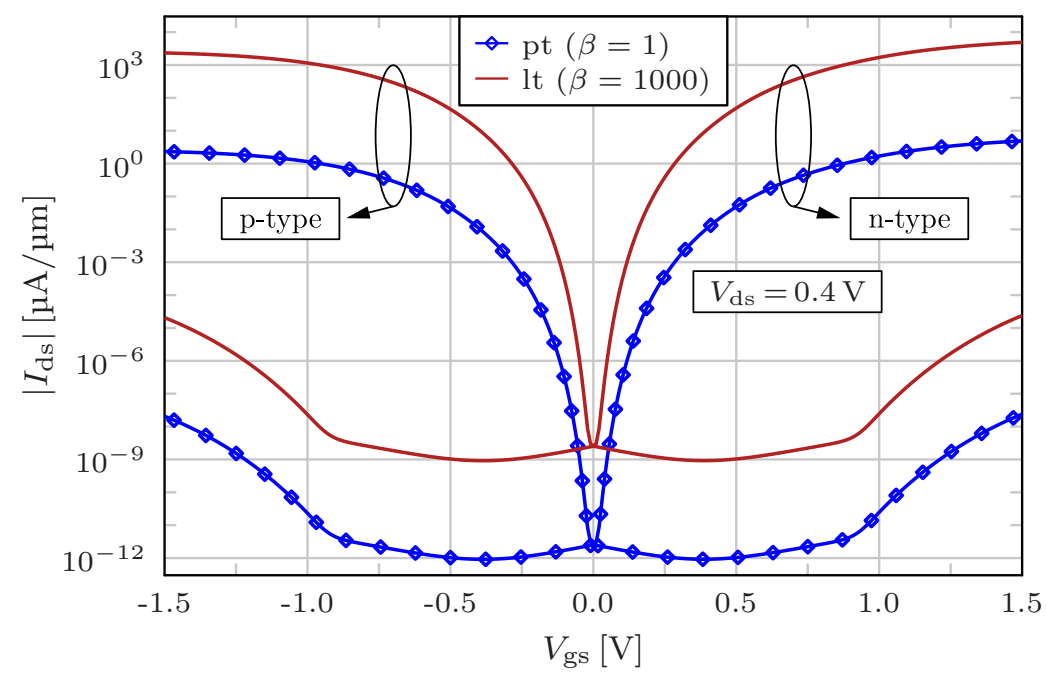

Figure 7.10: Transfer characteristic of the $n-$ and p-type SG line tunneling TFETs in comparison with the point tunneling transistors [62]. 
By keeping all these assumptions, the model is used to simulate a TFET-based inverter and then with the help of this inverter an 11-stage ring-oscillator is simulated. These simulations are done for both $\beta=1$ and $\beta=1000$. Figure 7.11 presents the VTC of the inverter on the left axis and the $I_{\text {out }}$ on the right axis. For $V_{\text {in }}=0 \mathrm{~V}$ and $V_{\mathrm{DD}}=0.4 \mathrm{~V}$ (see Fig. 7.4(a)), the p-type TFET is in on-state and n-type transistor is in its off-state. Thus, it can be said that the supply voltage with a low resistance is connected to the $V_{\text {out }}$ and therefore it is equal to $0.4 \mathrm{~V}$. By increasing the input voltage the $\mathrm{n}$-type TFET in the inverter also starts to conduct the current and switch to the on-state. As the result of the created path between $V_{\mathrm{DD}}$ and ground, the output current in this moment reaches its maximum. Finally, by further increment in $V_{\text {in }}$ the n-type transistors is situated in on-state and the p-type TFET is turned off. So, this time one can say that the supply voltage with a high resistance is connected to the output and hence $V_{\text {out }}=0 \mathrm{~V}$. Moreover, it can be seen that for the both point and line tunneling TFET, VTC shows similar slope. However, the inverter which is based on line tunneling TFET obviously $I_{\text {out }}$ is $\beta$ times higher.

From the explanations in Sec. 7.1 and by considering the clarifications in the previous paragraph, one could easily anticipate that the point tunneling ring-oscillator has a longer oscillation period in comparison to the line tunneling one. This effect is due to the lower on-current of the point tunneling device and is shown in Fig. 7.12 that $T$ in this case is almost equal to $15 \mathrm{~ns}$ while in line tunneling case $T \approx 15 \mathrm{ps}$.

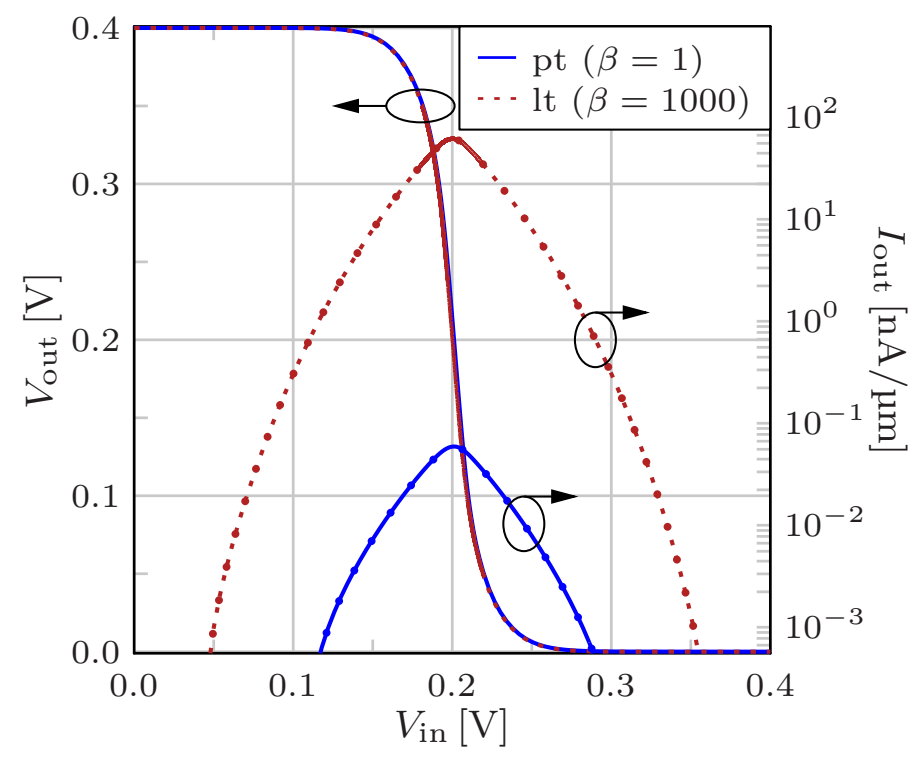

Figure 7.11: Voltage transfer curves of point tunneling and line tunneling inverters simulated in Cadence. The supply voltage is $V_{\mathrm{DD}}=0.4 \mathrm{~V}$, the solid lines refer to the point tunneling case and the dotted lines represent the line tunneling case [62]. 
Consequently, as it is illustrated in Fig. 7.13, the intrinsic delay of each inverter based on the point tunneling TFETs is three orders of magnitude larger than in one designed with the line tunneling transistors. Here $\tau$ is also calculated for different channel lengths. That is to say, the simulations of an 11-stage ring-oscillator for $l_{\mathrm{ch}}=22,45,65,90,180$ and $250 \mathrm{~nm}$ are done. Then, by extracting $T$ from these simulations and setting $n=11$ in Eq. (7.1), $\tau$ is calculated. These values are depicted in Fig. 7.13 with the symbols. Furthermore, similar to 7.8 , the relation between $\tau$ and $l_{\mathrm{ch}}$, which is approximated by means of Eq. (7.2), is illustrated with solid lines. It can be seen that the obtained values are in a good agreement with the theoretical estimations. According to the Eq. (7.2) and in consideration of the fact that the current in TFET is not a function of $l_{\mathrm{ch}}$, the intrinsic inverter delay is proportional to $l_{\mathrm{ch}}$ and both the data from simulations and the theoretical calculations support this concept. In addition, to have a reference for a comparison, the corresponding correlation for a CMOS inverter is also given (see the dotted lines in Fig. 7.13). Here it can be seen that $\tau$ is proportional to $l_{\mathrm{ch}}^{2}$ which is due to the dependency of both current and capacitance of CMOS to the length of its channel $[62]$.

The ring-oscillator simulations for TFET with $l_{\mathrm{ch}}=22 \mathrm{~nm}$ are repeated by varying $V_{\mathrm{dd}}$. From these simulations the average current in each case is extracted. Then, by having the voltage, current and number of inverters in the circuit the average power consumption of each inverter is calculated:

$$
P_{\mathrm{avg}}=\frac{I_{\mathrm{avg}} \cdot V_{\mathrm{DD}}}{n} .
$$

In Fig. 7.14 the intrinsic inverter delay on the left axis and $P_{\mathrm{avg}}$ of each inverter in the ring-oscillator circuit on the right axis as a function of the $V_{\mathrm{DD}}$ are illustrated. The line tunneling inverter in comparison to the point tunneling inverter has a shorter $\tau$ and bigger average power consumption. Furthermore, it can be seen that an increase of $V_{\mathrm{DD}}$ leads to a higher power consumption, but the intrinsic delay is not drastically affected. It indicates that an increment in TFET on-current caused by higher $V_{\mathrm{DD}}$ is likely to be compensated by a larger amount of charge carriers stored in the intrinsic capacitances and hence, not a significant change can be seen in $\tau$ [62].

Considering Fig. 7.13 and Fig. 7.14 it can be concluded that with the help of innovative structures of TFETs such as line tunneling, it is possible to improve the dynamic performance of these transistors into a level which can compete with CMOS technology yet with lower supply voltage. 


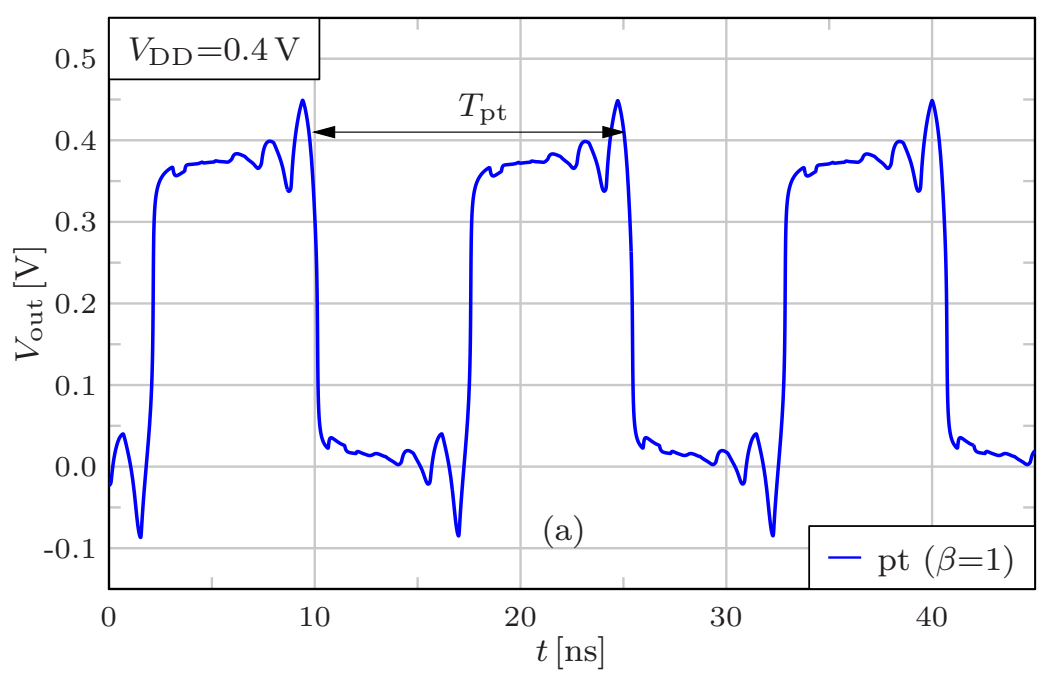

(a)

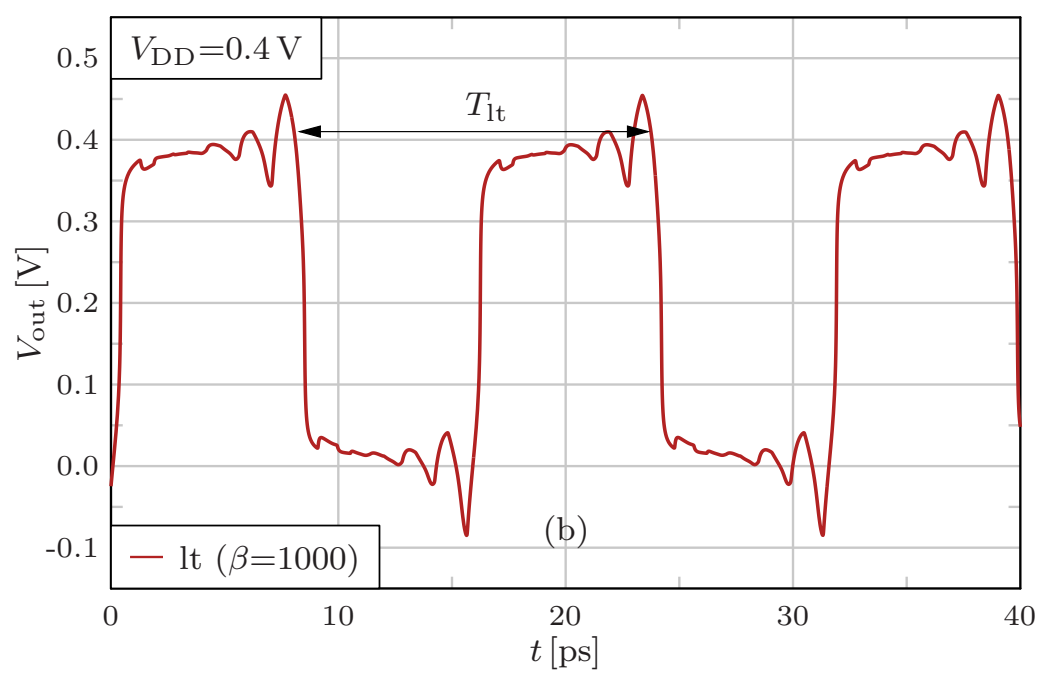

(b)

Figure 7.12: Output voltage of the 11-stage ring-oscillator in (a) point tunneling and (b) line tunneling case. $T_{\mathrm{pt}}$ presents the oscillation's period in [ns] for the point tunneling and $T_{\mathrm{lt}}$ refers to the oscillation period in [ps] for the line tunneling mode [62]. 


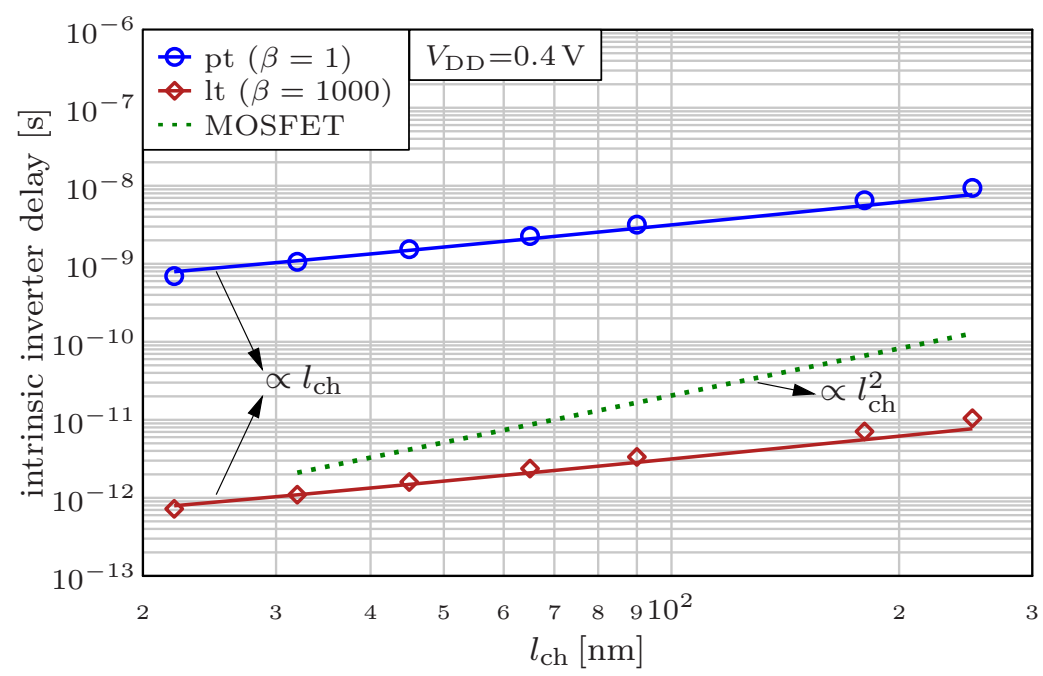

Figure 7.13: Intrinsic inverter delays, which are extracted from oscillation period of the 11stage ring-oscillator, for both point and line tunneling cases are shown with symbols. The solid lines represent the theoretical relation between of the intrinsic inverter delay and the $l_{\mathrm{ch}}$. The dotted line refers to the corresponding correlation for a CMOS inverter in the $32 \mathrm{~nm}$ process node with $V_{\mathrm{DD}}=0.8 \mathrm{~V}[62]$.

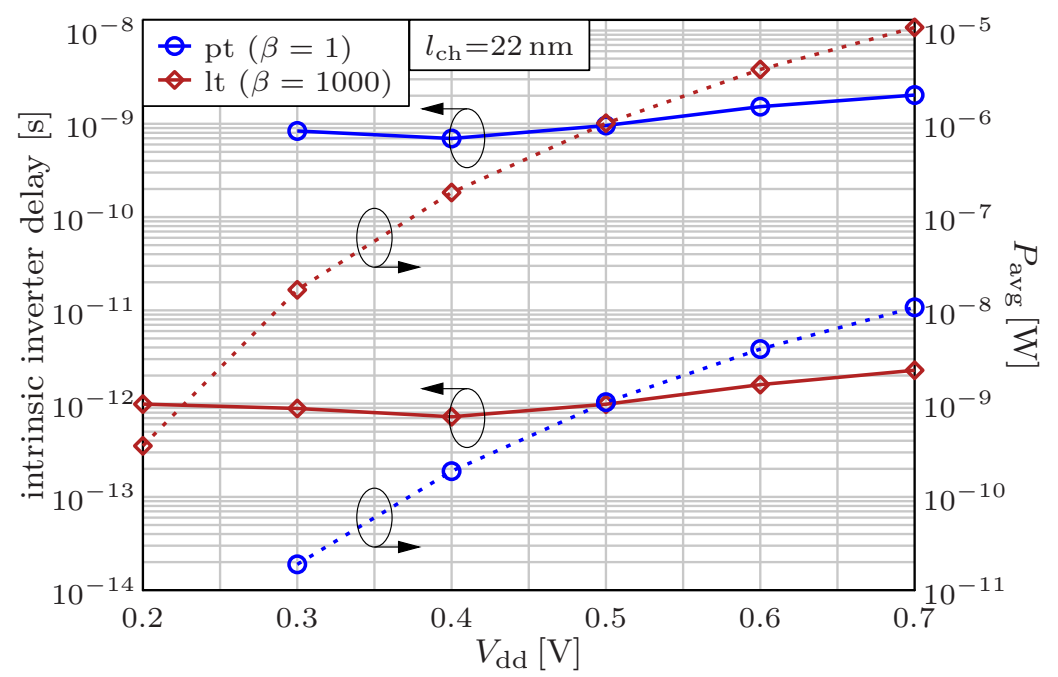

Figure 7.14: Intrinsic inverter delay and the average power consumption in an 11-stage ringoscillator are shown on the left and right axis, respectively, as a function of $V_{\mathrm{DD}}[62]$. 


\section{CHAPTER 8}

\section{Conclusion}

This dissertation presents a charge-based compact model for the intrinsic capacitances in TFETs. The model is based on a closed-form expression for the mobile charge in the channel introduced in $[57,69]$. In the following, a brief summary is given of the steps taken in the course of this project from development of the model to the circuit simulations and results. A conclusion is then drawn under consideration of the results that have finally been achieved.

First, the compact model for an n-type Si DG TFET was derived. Similar to the other suggested capacitance models by Zhang et al. in [39] and Lu et al. in [38], in the preliminary assumptions it has been considered that the inversion channel charges are equally distributed. To calculate the total mobile charge of the channel, it was therefore adequate to calculate the charge density at one point of the channel and then integrate it over the entire area. Although this assumption is not valid in a real structure, the resulted capacitances based on it were in a quite good agreement with the TCAD simulations. However, for further investigations the capacitance model was compared to the measured capacitances of a fabricated TFET. In the light of the measurement data, it has become clear that the assumption of a uniform charge distribution in the channel may work if the model is compared with the simulated data, but it is not sufficient to show all the details and gain a deep insight into the capacitive behavior of a real TFET. Hence, it has been concluded that some effects including the channel resistance and parasitic elements are needed to be integrated into the model.

The first series of measurements correspond to a p-type Si SG planar TFET which is fabricated in Juelich Forschungszentrum. In order to include the effect of the channel resistance, the compact DC model provided in [35] is used. That is to say, considering the structural parameters of the device which are under investigation and with the help of the DC model, first $I_{\mathrm{ds}}$ is calculated. Then, the bias dependent channel resistivity by means of an empirical method is calculated. Having $I_{\mathrm{ds}}$ and $R_{\mathrm{ch}}$ allows for calculating the voltage drop between the junction and the tunneling barrier in the channel. Therefore, by applying the $V_{\mathrm{ds}}$ into the charge density closed-form expression, the mobile charge density at the drain junction is obtained and by implementing $V_{\mathrm{ds}}-\Delta V$ in the aforementioned formula, the mobile charge 
per gate area at the tunneling barrier in the channel close to the source junction is calculated. Then by taking the average of the two obtained values and multiplying the result with the area of the channel, the total mobile charge of the channel in the on-state is achieved. The compact intrinsic capacitance model considers not only the on-state, but also the ambipolar-state of the transistor. Hence, in the ambipolar-state to calculate the charge density at the junction and tunneling barrier located close to the drain junction, respectively $V_{\mathrm{s}}$ and $V_{\mathrm{s}}-\Delta V$ are used in the charge density expression.

Adding the effect of the channel resistance into the intrinsic capacitance model by combining it with the compact DC model has brought the results of the model closer to the measurement data. Moreover, due to the fact that the DC model takes into account the effect of traps and the doping concentration in the source and drain regions, the capacitance model can also capture the influence of these parameters on the intrinsic capacitances of TFET. Nevertheless, the capacitive behavior of the fabricated TFET could still not be fully reproduced by model. Hence, it has been decided to incorporate the parasitic effect of series resistances into the capacitance model.

To do so, it is considered that a resistance is connected in series at each terminal on the source and drain side. The effect of these resistances on the intrinsic capacitances is implemented with the same method which is introduced in [73]. This approach has been originally developed to enhance the compact model of MOSFETs, though is appropriate for other structures as well. But, after all, the model needed some more details to show the similar behavior of the measured capacitances.

Eventually, these measurement data have led to investigations into the influence of the Schottky barrier at the the source and drain terminals on the intrinsic capacitances of the TFET and to the inclusion of this effect in the compact model. For this purpose an empirical method is used. This method has evolved on the basis of the analysis of experimental data and shows that in the on-state at higher $\left|V_{\mathrm{gs}}\right|$ the capacitive behavior of the transistor is assigned not only to $C_{\mathrm{gd}}$ but also partially to $C_{\mathrm{gs}}$. In the ambipolar-state illustrates that for higher $\left|V_{\mathrm{gs}}\right|$, the intrinsic capacitance of the TFET is not identical to the $C_{\mathrm{gs}}$ however, a sum of both capacitances $C_{\mathrm{gs}}$ and $C_{\mathrm{gd}}$.

The results of the TCAD simulations of the planar structure including Schottky barriers at the terminals could not fulfill the expectation and reproduce a similar capacitive behavior as in measurements. Different approaches have been considered to evaluate the correct values and ratios, with which at a certain gate bias one capacitance decreases and the other increases. In the end, with the help of TCAD Sentaurus, some Schottky diodes with different drain doping concentrations are simulated. By comparing their current with the measured $I_{\mathrm{ds}}$ in a p-type TFET, the diode with a current closer to $I_{\mathrm{ds}}$ is chosen and its conductance is extracted. This value is used to estimate the small signal conductance of the Schottky barrier in the TFET and check the effect on the intrinsic capacitances. Finally, the model could show a similar pattern as in the measured data.

As the last step of verifying the model, it is compared to the measurement data of an 
n-type Si NW TFET. This structure is fabricated by imec, Belgium [83] and the measurements are provided by University of Sao Paulo, Brazil. Considering the cylindrical shape of the NW TFET, an equivalent DG $l_{\mathrm{ch}}$ is used in the model. Furthermore, to cover the effect of the gate-source overlap in this structure, a constant parallel capacitance to the $C_{\text {eff }}$ is implemented in the compact model. Then, with the help of the other already existing fitting parameters, the model is fitted to measurements. Comparing the results to the measured transfer characteristic and capacitances of the NW shows that the model is in a good agreement with the experimental data.

After completion of the model, it is transferred to the Verilog-A language to enable circuit simulations. Using this compact TFET model, an inverter circuit is designed in Cadence Virtuoso. This circuit is then used to design a ring-oscillator. In order to examine the effect of the device parameters on the behavior of circuits, the simulations are performed using different parameters and properties for both $\mathrm{p}-$ and n-type TFETs. In each case, to ensure that both the DC and AC simulations of these transistors as a single element are reliable, firstly the results of the compact model simulations of the TFETs are verified against TCAD simulations. The circuit simulations based on verified transistors are then performed. The parameters which are considered in these simulations are: $N_{\mathrm{t}}, N_{\mathrm{d}}, l_{\mathrm{ch}}$ and $V_{\mathrm{fb}}$. Although all the mentioned parameters affect the behavior of the device, only by varying $V_{\mathrm{fb}}$ a significant influence in outputs of the circuits has been observed which is due to the operation range of the circuits. Based on these observations, two main setups for the circuit simulations are taken into account: HP and LP mode. In HP mode, $V_{\mathrm{fb}}$ is shifted by $-200 \mathrm{mV}$. As a result, the transfer characteristic of the TFET is shifted and it appears as having a higher on-current and thus greater performance. LP refers to the case in which $V_{\mathrm{fb}}-\mathrm{shift}=50 \mathrm{mV}$. In contrast to the HP mode, in this case the shift in $V_{\mathrm{fb}}$ yields to a lower $I_{\mathrm{ds}}$ for low $V_{\mathrm{gs}}$.

Comparing the inverter output in HP and LP mode shows that the inverter in LP case has a higher DC gain and lower switching current in comparison to the HP mode. Hence, the transient simulations of the inverter for a pulse input demonstrate higher overshoot and undershoot in LP mode than in HP. Moreover, the 3-stage ring-oscillator in LP case has a lower oscillation frequency in comparison to the HP setup.

Next, by varying $l_{\mathrm{ch}}$ of the TFETs, the ring-oscillator simulations are performed in both HP and LP mode. With the aid of these simulations, first the oscillation period is calculated in each case and then by means of $T$, intrinsic inverter delay is obtained. For comparison purpose, also the intrinsic inverter delay of a CMOS inverter as a function of its channel length is considered.

Bearing in mind the fact that with further innovation of the structures, the TFET on-current can be enhanced, the $I_{\mathrm{ds}}$ [92], further circuit simulations are carried out to observe the behavior of TFET-based circuits in case they have a higher current. In this regard, output of the circuits based on the point tunneling TFET are compared to those based on line tunneling TFET. To do so, the compact model is firstly verified against TCAD simulations of a SG n-type TFET as well as p-type TFET. Therefore, the model is fitted for an ideal line tunneling case in which 
the on-current of the TFET is 1000 times higher than in the point tunneling case. Afterwards, simulations of an inverter and a 11-stage ring-oscillator are performed to compare the point and line tunneling setups.

By means of these simulations the oscillation period of the ring-oscillator and from that the intrinsic inverter delay as a function of $l_{\mathrm{ch}}$ is obtained. The resulted $\tau$ for the point tunneling and line tunneling case are compared to each other and also to the corresponding data of a CMOS inverter. Finally, the average power consumption of the ring-oscillator by varying $V_{\mathrm{DD}}$ was achieved.

After all mentioned above and considering the results of the circuit simulation, the following can be concluded:

- The intrinsic capacitance model is flexible and scalable and can be employed for various structures. In the course of the model verification it is shown, despite that the model is designed based on a short-channel Si DG n-type structure, its simplicity and flexibility allows for calculating of various structures and also different types such as the p-type SG planar TFET and the n-type NW TFET.

- The model is applicable for a wide range of $V_{\mathrm{gs}}$ and considers not only the on-state of the device but also the off- and ambipolar-state.

- It is accurate. The compact model is validated using both TCAD simulation and measurement data. The results of the model show a good match to those obtained by TCAD simulation and also to the measurements.

- It considers the parasitic effects. The parasitic elements can drastically affect the capacitive behavior of the TFETs. Hence, it is important to include them in the model.

- The model can also include the non-ideal effects such as TAT on the capacitances.

- From all these points, it can be concluded that the compact model can provide a reliable insight about the intrinsic capacitances in TFET.

- Furthermore, the compact model presented here is not time consuming or computationally demanding which allows for circuit simulations including multiple TFETs.

- The compact TFET model is SPICE friendly.

- Although the variation of $N_{\mathrm{t}}$ and $N_{\mathrm{d}}$ has a strong influence on the electrical characteristics of the TFET considered as a single element, no such apparent difference is evident in the circuit simulation results.

- Varying $V_{\mathrm{fb}}$ has a major impact on the performance of the TFET-based circuits. In fact, by changing $V_{\mathrm{fb}}$ the transfer characteristics of the TFET is shifted. Depending on the direction of this shift, the TFET shows higher or lower current for smaller $V_{\mathrm{gs}}$ which 
is similar to the situation that the on-current of the transistor is enhanced or reduced. Thus, the variation of the parameter $V_{\mathrm{fb}}$ leads to a drastic change of the output on the circuit level.

- In TFET inverter $\tau$ is proportional to $l_{\mathrm{ch}}$ whereas for standard MOSFET it is proportional to $l_{\mathrm{ch}}^{2}$, which is due to the fact that in TFET the current is not a function of $l_{\mathrm{ch}}$.

- To improve the dynamic performance of the TFET-based circuits, the on-current of the TFET needs to be increased.

- By increasing $V_{\mathrm{DD}}$ the power consumption in the TFET and consequently the TFET-based inverter goes higher, but the intrinsic inverter delay is not affected.

- There are still challenges that must be tackled before the TFETs can really meet all expectations and conquer the ICs. Researchers are constantly trying out new manufacturing methods, materials and structures to push the limits. With each step, new aspects of the TFET become apparent. In this case, fitting parameters can help to adapt the model and make it work without applying any complicated changes in the model.

- Once the obstacles which limit the on-current of TFET are overcome with the help of novel structures and materials, TFET will be able to stand up to the competition with CMOS technology, however with considerably lower supply voltage requirements.

- There are still factors such as temperature and frequency which have an effect on the capacitive behavior of the TFET and consequently on the output of the circuit which are not considered in this model. Probably in future works they can be included in the compact model as well. 


\section{References}

[1] N. Arora, MOSFET Modeling for VLSI Simulation. World Scientific Publishing Company, 1993.

[2] D. J. Frank, R. H. Dennard, E. Nowak, P. M. Solomon, Y. Taur, and H.-S. P. Wong, "Device scaling limits of Si MOSFETs and their application dependencies," Proceedings of the IEEE, vol. 89, no. 3, pp. 259-288, 2001.

[3] R. H. Dennard, F. H. Gaensslen, V. L. Rideout, E. Bassous, and A. R. LeBlanc, "Design of ion-implanted MOSFET's with very small physical dimensions," IEEE Journal of Solid-State Circuits, vol. 9, no. 5, pp. 256-268, 1974.

[4] S. Thompson, M. Alavi, M. Hussein, P. Jacob, C. Kenyon, P. Moon, M. Prince, S. Sivakumar, S. Tyagi, and M. Bohr, "130nm logic technology featuring 60nm transistors, low-k dielectrics, and cu interconnects.," Intel Technology Journal, vol. 6, no. 2, 2002.

[5] K. P. Cheung, "On the $60 \mathrm{mV} /$ dec@ $300 \mathrm{~K}$ limit for MOSFET subthreshold swing," in Proceedings of 2010 International Symposium on VLSI Technology, System and Application, pp. 72-73, IEEE, 2010.

[6] K. Mistry, C. Allen, A. Beattie, et al., "A 45nm logic technology with high-k+ metal gate transistors," Strained Silicon, vol. 9, pp. 247-250, 2007.

[7] J. K. Mamidala, R. Vishnoi, and P. Pandey, Tunnel field-effect transistors (TFET): modelling and simulation. John Wiley \& Sons, 2016.

[8] A. Klös, Nanoelektronik: Bauelemente der Zukunft. Carl Hanser Verlag GmbH Co KG, 2018.

[9] D. J. Frank, R. H. Dennard, E. Nowak, P. M. Solomon, Y. Taur, and H.-S. P. Wong, "Device scaling limits of Si MOSFETs and their application dependencies," Proceedings of the IEEE, vol. 89, no. 3, pp. 259-288, 2001.

[10] B. Iñiguez, T. A. Fjeldly, A. Lázaro, F. Danneville, and M. J. Deen, "Compact-modeling solutions for nanoscale double-gate and gate-all-around MOSFETs," IEEE Transactions on electron devices, vol. 53, no. 9, pp. 2128-2142, 2006. 
[11] G. Y. Krasnikov, N. Zaitsev, and I. Matyushkin, "On the issue of an equivalent oxide thickness evaluation in nanoscale MISFETs," Russian Microelectronics, vol. 40, no. 1, pp. 25-30, 2011.

[12] J.-P. Colinge, "Multiple-gate soi mosfets," Solid-state electronics, vol. 48, no. 6, pp. 897-905, 2004.

[13] D. Jiménez, J. J. Sáenz, B. Inıquez, J. Suñé, L. Marsal, and J. Pallarès, "Unified compact model for the ballistic quantum wire and quantum well metal-oxide-semiconductor fieldeffect-transistor," Journal of Applied Physics, vol. 94, no. 2, pp. 1061-1068, 2003.

[14] K. Fukuda, T. Mori, W. Mizubayashi, Y. Morita, A. Tanabe, M. Masahara, T. Yasuda, S. Migita, and H. Ota, "A compact model for tunnel field-effect transistors incorporating nonlocal band-to-band tunneling," Journal of Applied Physics, vol. 114, no. 14, p. 144512, 2013.

[15] A. C. Seabaugh and Q. Zhang, "Low-voltage tunnel transistors for beyond CMOS logic," Proceedings of the IEEE, vol. 98, no. 12, pp. 2095-2110, 2010.

[16] P.-F. Wang, K. Hilsenbeck, T. Nirschl, M. Oswald, C. Stepper, M. Weis, D. SchmittLandsiedel, and W. Hansch, "Complementary tunneling transistor for low power application," Solid-State Electronics, vol. 48, no. 12, pp. 2281-2286, 2004.

[17] J. Appenzeller, Y.-M. Lin, J. Knoch, and P. Avouris, "Band-to-band tunneling in carbon nanotube field-effect transistors," Physical review letters, vol. 93, no. 19, p. 196805, 2004.

[18] A. M. Ionescu and H. Riel, "Tunnel field-effect transistors as energy-efficient electronic switches," nature, vol. 479, no. 7373, pp. 329-337, 2011.

[19] D. Sarkar, X. Xie, W. Liu, W. Cao, J. Kang, Y. Gong, S. Kraemer, P. M. Ajayan, and K. Banerjee, "A subthermionic tunnel field-effect transistor with an atomically thin channel," Nature, vol. 526, no. 7571, pp. 91-95, 2015.

[20] D. Verreck, A. S. Verhulst, K.-H. Kao, W. G. Vandenberghe, K. De Meyer, and G. Groeseneken, "Quantum mechanical performance predictions of pnin versus pocketed line tunnel field-effect transistors," IEEE transactions on electron devices, vol. 60, no. 7, pp. 2128-2134, 2013.

[21] G. Dewey, B. Chu-Kung, J. Boardman, J. Fastenau, J. Kavalieros, R. Kotlyar, W. Liu, D. Lubyshev, M. Metz, N. Mukherjee, et al., "Fabrication, characterization, and physics of III-V heterojunction tunneling field effect transistors (H-TFET) for steep sub-threshold swing," in 2011 International electron devices meeting, pp. 33-6, IEEE, 2011. 
[22] L. Knoll, Q.-T. Zhao, A. Nichau, S. Trellenkamp, S. Richter, A. Schäfer, D. Esseni, L. Selmi, K. K. Bourdelle, and S. Mantl, "Inverters with strained Si nanowire complementary tunnel field-effect transistors," IEEE electron device letters, vol. 34, no. 6, pp. 813-815, 2013.

[23] E. Memisevic, J. Svensson, E. Lind, and L.-E. Wernersson, "InAs/InGaAsSb/GaSb nanowire tunnel field-effect transistors," IEEE Transactions on Electron Devices, vol. 64, no. 11 , pp. $4746-4751,2017$.

[24] Synopsys, Inc., Sentaurus ${ }^{T M}$ Device User Guide. Version O-2018.06, June 2018.

[25] SILVACO, Inc., "ALTAS User's Manual Device Simulation Software," September 2010.

[26] Global TCAD Solutions GmbH Landhausgasse 4/1a, 1010 Wien Austria, VSP-a quantumelectronic simulation framework. Springer Science+Business Media, 2013.

[27] I. Crosslight Software, "Crosslight," 2020. https://crosslight.com.

[28] Cogenda Pte Ltd., VisualTCAD User's Guide, Sept. 2017. Version 1.7.2.

[29] C. McAndrew, "Compact modeling: Principles, techniques, and applications," in Statistical Modeling using Backward Propagation of Variance, Springer New York, 2010.

[30] L. Zhang and M. Chan, "Spice modeling of double-gate tunnel-fets including channel transports," IEEE Transactions on Electron Devices, vol. 61, no. 2, pp. 300-307, 2014.

[31] A. Biswas, L. De Michielis, A. Bazigos, and A. M. Ionescu, "Compact modeling of DGTunnel FET for Verilog-A implementation," in 2015 45th European Solid State Device Research Conference (ESSDERC), pp. 40-43, IEEE, 2015.

[32] M. Graef, F. Hosenfeld, F. Horst, A. Farokhnejad, F. Hain, B. Iñíguez, and A. Kloes, "Advanced analytical modeling of double-gate Tunnel-FETs-A performance evaluation," Solid-State Electronics, vol. 141, pp. 31-39, 2018.

[33] M. Graef, F. Hain, F. Hosenfeld, F. Horst, A. Farokhnejad, B. Iniguez, and A. Kloes, "Numerical analysis and analytical modeling of RDF in DG Tunnel-FETs," in 2016 Joint International EUROSOI Workshop and International Conference on Ultimate Integration on Silicon (EUROSOI-ULIS), pp. 64-67, IEEE, 2016.

[34] M. Graef, F. Hain, F. Hosenfeld, B. Iniguez, and A. Kloes, "Analytical approach to consider gaussian junction profiles in compact models of tunnel-FETs," in EUROSOI-ULIS 2015: 2015 Joint International EUROSOI Workshop and International Conference on Ultimate Integration on Silicon, pp. 213-216, IEEE, 2015.

[35] F. Horst, Compact DC Modeling of Tunnel-FETs. PhD thesis, Universitat Rovira i Virgili, 2019 . 
[36] Y. Yang, X. Tong, L.-T. Yang, P.-F. Guo, L. Fan, and Y.-C. Yeo, "Tunneling field-effect transistor: Capacitance components and modeling," IEEE Electron Device Letters, vol. 31, no. 7 , pp. $752-754,2010$.

[37] Y. Cheng and C. Hu, MOSFET modeling \& BSIM3 user's guide. Springer Science \& Business Media, 1999.

[38] B. Lu, H. Lu, Y. Zhang, Y. Zhang, X. Cui, Z. Lv, S. Yang, and C. Liu, "A chargebased capacitance model for double-gate tunnel FETs with closed-form solution," IEEE Transactions on Electron Devices, vol. 65, no. 1, pp. 299-307, 2017.

[39] L. Zhang, X. Lin, J. He, and M. Chan, "An analytical charge model for double-gate tunnel fets," IEEE transactions on electron devices, vol. 59, no. 12, pp. 3217-3223, 2012.

[40] L. Zhang, J. He, and M. Chan, "A compact model for double-gate tunneling field-effecttransistors and its implications on circuit behaviors," in 2012 International Electron Devices Meeting, pp. 6-8, IEEE, 2012.

[41] H. Lu, W. Li, Y. Lu, P. Fay, T. Ytterdal, and A. Seabaugh, "Universal charge-conserving TFET SPICE model incorporating gate current and noise," IEEE Journal on Exploratory Solid-State Computational Devices and Circuits, vol. 2, pp. 20-27, 2016.

[42] B. J. Copeland, "The modern history of computing," in The Stanford Encyclopedia of Philosophy (E. N. Zalta, ed.), Metaphysics Research Lab, Stanford University, winter 2017 ed., 2017.

[43] D. Swade, "The Babbage Engine," 1991. http://www.computerhistory.org/babbage/history, Accessed: 21.01.2019.

[44] “Science Museum Group." http://collection.sciencemuseum.org.uk/objects/co62243/differenceengine-no-1-difference-engine-portion-only, Accessed: 21.01.2019.

[45] E. B. Derek Cheung, Conquering the Electron: The Geniuses, Visionaries, Egomaniacs, and Scoundrels Who Built Our Electronic Age. ROWMAN \& LITTLEFIELD, 2014.

[46] "ENIAC," circa 1947 to 1955. https://commons.wikimedia.org/wiki/File:Eniac.jpg, Accessed: 21.01.2019.

[47] G. E. Moore, "Cramming more components onto integrated circuits, reprinted from electronics, volume 38, number 8, april 19, 1965, pp. 114 ff.," IEEE Solid-State Circuits Society Newsletter, vol. 11, no. 3, pp. 33-35, 2006.

[48] M. Roser and H. Ritchie, "Technological progress," Our World in Data, 2019. https://ourworldindata.org/technological-progress. 
[49] S. M. Sze and K. K. Ng, Physics of Semiconductor Devices. John Wiley \& Sons, Inc., 2007.

[50] F. Thuselt, Physik der Halbleiterbauelemente. Springer-Verlag Berlin Heidelberg 2011, 2011.

[51] "Encyclopaedia britannica," January 25, 2019. https://www.britannica.com/science/semiconductor, Accessed: 21.02.2019.

[52] "Semiconductors. Brilliant.org. Retrieved 16:47." https://brilliant.org/wiki/semiconductors/, Accessed: 2019-11-25.

[53] D. J. Griffiths, Introduction to Quantum Mechanics. Prentice Hall, 1994.

[54] M. Schwarz, Two-dimensional analytical predictive modeling of schottky barrier SOI and multi-gate MOSFETs. PhD thesis, 2012.

[55] E. Weber, Electromagnetic Fields: Theory and Applications. No. 1 in Electromagnetic Fields, Polytechnic Institute of Brookly: Wiley, 3 ed., April 1950.

[56] K. KÃ $1 / 4 \operatorname{pfm} \tilde{A}^{1} 1 / 4 l$ ler, W. Mathis, and A. Reibiger, Theoretische Elektrotechnik, vol. 17. Springer Berlin Heidelberg New York, 2006.

[57] T. Holtij et al., ANALYTICAL COMPACT MODELING OF NANOSCALE MULTIPLEGATE MOSFETS. PhD thesis, Universitat Rovira i Virgili, 2014.

[58] R. M. Corless, G. H. Gonnet, D. E. Hare, D. J. Jeffrey, and D. E. Knuth, "On the lambertw function," Advances in Computational mathematics, vol. 5, no. 1, pp. 329-359, 1996.

[59] Mathworks, Inc., Matlab. 2019. Version R2019a.

[60] E. W. Weisstein, "Lambert w-function."

[61] D. Verreck, G. Groeseneken, and A. Verhulst, "The tunnel field-effect transistor," Wiley Encyclopedia of Electrical and Electronics Engineering, pp. 1-24, 1999.

[62] A. Farokhnejad, F. Horst, A. Kloes, B. Iñíguez, and F. Lime, "Impact of on-current on the static and dynamic performance of TFET inverters," in 2019 IEEE SOI-3D-Subthreshold Microelectronics Technology Unified Conference (S3S), IEEE, Oct. 2019. (Accepted for Publication).

[63] S. Glass, K. Kato, L. Kibkalo, J.-M. Hartmann, S. Takagi, D. Buca, S. Mantl, and Z. Qing-Tai, "A novel gate-normal tunneling field-effect transistor with dual-metal gate," IEEE Journal of the Electron Devices Society, vol. 6, pp. 1070-1076, 2018. 
[64] D. B. Abdi and M. J. Kumar, "Controlling ambipolar current in tunneling FETs using overlapping gate-on-drain," IEEE Journal of the Electron Devices Society, vol. 2, no. 6, pp. 187-190, 2014.

[65] M. H. Ben-Jamaa, K. Mohanram, and G. De Micheli, "An efficient gate library for ambipolar CNTFET logic," IEEE Transactions on Computer-Aided Design of Integrated Circuits and Systems, vol. 30, no. 2, pp. 242-255, 2011.

[66] X. Yang and K. Mohanram, "Ambipolar electronics," 2010.

[67] A. Farokhnejad, M. Schwarz, M. Graef, F. Horst, B. Iñíguez, F. Lime, and A. Kloes, "Effect of schottky barrier contacts on measured capacitances in tunnel-FETs," in 2018 Joint International EUROSOI Workshop and International Conference on Ultimate Integration on Silicon (EUROSOI-ULIS), pp. 1-4, IEEE, 2018.

[68] C. Wu, R. Huang, Q. Huang, C. Wang, J. Wang, and Y. Wang, "An analytical surface potential model accounting for the dual-modulation effects in tunnel FETs," IEEE Transactions on Electron Devices, vol. 61, no. 8, pp. 2690-2696, 2014.

[69] A. Kloes, M. Schwarz, T. Holtij, and A. Navas, "Quantum confinement and volume inversion in MOS3 model for short-channel Tri-Gate MOSFETs," IEEE Transactions on Electron Devices, vol. 60, pp. 2691-2694, aug 2013.

[70] A. Kloes and A. Kostka, "A new analytical method of solving $2 \mathrm{~d}$ poisson's equation in mos devices applied to threshold voltage and subthreshold modeling," Solid-State Electronics, vol. 39, no. 12, pp. 1761-1775, 1996.

[71] A. Kloes, M. Schwarz, and T. Holtij, "MOS ${ }^{3}$ : A new physics-based explicit compact model for lightly doped short-channel triple-gate SOI MOSFETs," IEEE transactions on electron devices, vol. 59, no. 2, pp. 349-358, 2012.

[72] A. Kloes, M. Weidemann, and M. Schwarz, "Analytical current equation for short channel SOI multigate FETs including 3D effects," Solid-state electronics, vol. 54, no. 11, pp. 14081415,2010 .

[73] T. Smedes and F. Klaassen, "Influence of channel series resistances on dynamic mosfet behaviour," Solid-state electronics, vol. 37, no. 2, pp. 251-254, 1994.

[74] A. Farokhnejad, M. Graef, F. Horst, C. Liu, Q. Zhao, B. Iñíguez, F. Lime, and A. Kloes, "Compact modeling of intrinsic capacitances in double-gate tunnel-fets," in 2017 Joint International EUROSOI Workshop and International Conference on Ultimate Integration on Silicon (EUROSOI-ULIS), pp. 140-143, IEEE, 2017. 
[75] C. Liu, Q. Han, S. Glass, G. V. Luong, K. Narimani, A. T. Tiedemann, A. Fox, W. Yu, X. Wang, S. Mantl, et al., "Experimental $i-v(t)$ and $c-v$ analysis of si planar p-tfets on ultrathin body," IEEE Transactions on Electron Devices, vol. 63, no. 12, pp. 5036-5040, 2016.

[76] A. Farokhnejad, M. Schwarz, F. Horst, B. Iñíguez, F. Lime, and A. Kloes, "Analytical modeling of capacitances in tunnel-FETs including the effect of schottky barrier contacts," Solid-State Electronics, vol. 159, pp. 191-196, 2019.

[77] J. Slotboom and H. De Graaff, "Measurements of bandgap narrowing in Si bipolar transistors," Solid-State Electronics, vol. 19, no. 10, pp. 857-862, 1976.

[78] J. D. Alamo, S. Swirhun, and R. Swanson, "Simultaneous measurement of hole lifetime, hole mobility and bandgap narrowing in heavily doped n-type silicon," Electron Devices Meeting, pp. 290-293, 1985.

[79] S. Feste, J. Knoch, D. Buca, Q. Zhao, U. Breuer, and S. Mantl, "Formation of steep, low schottky-barrier contacts by dopant segregation during nickel silicidation," Journal of applied physics, vol. 107, no. 4, p. 044510, 2010.

[80] Q.-T. Zhao, S. Richter, C. Schulte-Braucks, L. Knoll, S. Blaeser, G. V. Luong, S. Trellenkamp, A. Schäfer, A. Tiedemann, J.-M. Hartmann, et al., "Strained Si and SiGe nanowire tunnel FETs for logic and analog applications," IEEE journal of the Electron Devices Society, vol. 3, no. 3, pp. 103-114, 2015.

[81] M. Björk, J. Knoch, H. Schmid, H. Riel, and W. Riess, "Silicon nanowire tunneling field-effect transistors," Applied Physics Letters, vol. 92, no. 19, p. 193504, 2008.

[82] T. Vasen, P. Ramvall, A. Afzalian, G. Doornbos, M. Holland, C. Thelander, K. Dick, L.-E. Wernersson, and M. Passlack, "Vertical gate-all-around nanowire GaSb-InAs core-shell n-type tunnel FETs," Scientific reports, vol. 9, no. 1, pp. 1-9, 2019.

[83] A. Vandooren, D. Leonelli, R. Rooyackers, K. Arstila, G. Groeseneken, and C. Huyghebaert, "Impact of process and geometrical parameters on the electrical characteristics of vertical nanowire silicon n-TFETs," Solid-State Electronics, vol. 72, pp. 82-87, 2012.

[84] A. d. M. Nogueira, P. G. Agopian, and J. A. Martino, "Silicon nanowire Tunnel-FET differential amplifier using Verilog-A lookup table approach," in 2019 34th Symposium on Microelectronics Technology and Devices (SBMicro), pp. 1-4, IEEE, 2019.

[85] A. d. M. Nogueira, P. G. Agopian, J. A. Martino, E. Simoen, R. Rooyackers, C. Claeys, and N. Collaert, "OTA performance comparison designed with experimental NW-MOSFET and NW-TFET devices," in 2019 IEEE SOI-3D-Subthreshold Microelectronics Technology Unified Conference (S3S), San Jose, USA, Oct, IEEE. (in press). 
[86] T. Ma, S. A. Campbell, R. Smith, N. Hoilien, B. He, W. L. Gladfelter, C. Hobbs, D. Buchanan, C. Taylor, M. Gribelyuk, et al., "Group IVB metal oxides high permittivity gate insulators deposited from anhydrous metal nitrates," IEEE Transactions on Electron Devices, vol. 48, no. 10, pp. 2348-2356, 2001.

[87] K. Yılmaz, G. Darbandy, B. Iñíguez, F. Lime, and A. Kloes, "Equivalent length concept for compact modeling of short-channel GAA and DG MOSFETs," in 2019 Joint International EUROSOI Workshop and International Conference on Ultimate Integration on Silicon (EUROSOI-ULIS), pp. 1-4, IEEE, 2019.

[88] A. Farokhnejad, F. Horst, B. Iñíguez, F. Lime, and A. Kloes, "Evaluation of static/transient performance of TFET inverter regarding device parameters using a compact model," in ESSDERC 2019-49th European Solid-State Device Research Conference (ESSDERC), pp. 202-205, IEEE, 2019.

[89] Cadence Design Systems Inc., Virtuoso® Analog Design Environment. 2013. Version IC6.1.6.

[90] C. Sahu and J. Singh, "Device and circuit performance analysis of double gate junctionless transistors atl g=18 nm," The Journal of Engineering, vol. 2014, no. 3, pp. 105-110, 2014.

[91] "International technology roadmap for semiconductors 2.0 (itrs)," 2015.

[92] J.-T. Lin, T.-C. Wang, W.-H. Lee, C.-T. Yeh, S. Glass, and Q.-T. Zhao, "Characteristics of recessed-gate TFETs with line tunneling," IEEE Transactions on Electron Devices, vol. 65, pp. 769-775, feb 2018. 


\section{UNIVERSITAT ROVIRA I VIRGILI}

COMPACT MODELING OF INTRINSIC CAPACITANCES IN DOUBLE-GATE TUNNEL-FETS

Atieh Farokhnejad 


\section{UNIVERSITAT ROVIRA I VIRGILI}

COMPACT MODELING OF INTRINSIC CAPACITANCES IN DOUBLE-GATE TUNNEL-FETS

Atieh Farokhnejad 
COMPACT MODELING OF INTRINSIC CAPACITANCES IN DOUBLE-GATE TUNNEL-FETS

Atieh Farokhnejad

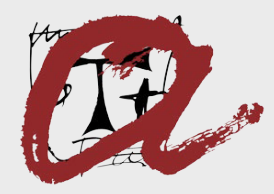

UNIVERSITAT

ROVIRA i VIRGILI 